\title{
IDEALISED MODELLING OF STORM SURGES IN LARGE-SCALE COASTAL BASINS
}

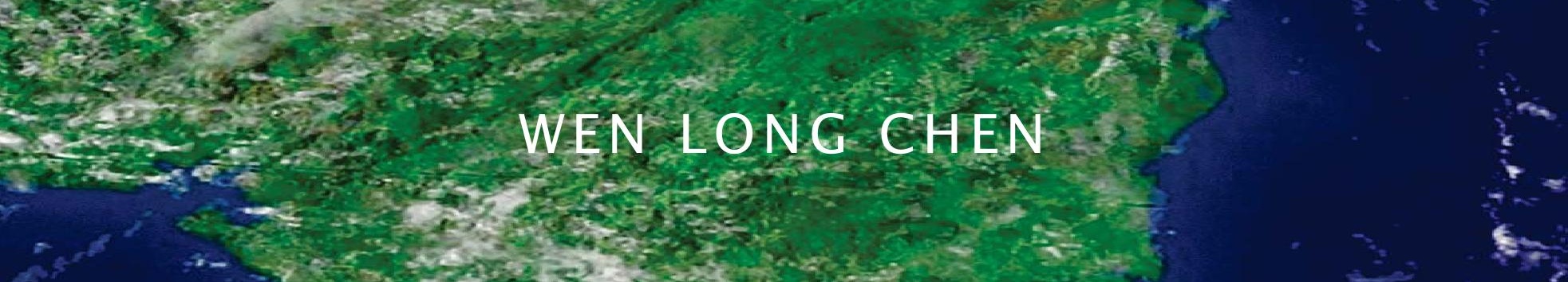


IDEALISED MODELLING OF STORM SURGES IN

LARGE-SCALE COASTAL BASINS 
Promotion committee:

Prof. dr. ir. J. van Amerongen University of Twente, chairman and secretary

Prof. dr. S.J.M.H. Hulscher

University of Twente, promotor

Dr. ir. P.C. Roos

University of Twente, co-promotor

Dr. ir. H.M. Schuttelaars

Prof. dr. ir. H.J. de Vriend

Delft University of Technology, co-promotor

University of Twente

Prof. dr. ir. C.H. Venner

University of Twente

Prof. dr. H.E. de Swart

Utrecht University

Prof. dr. ir. Z.B. Wang

Delft University of Technology

Dr. ir. M. van Ledden

Delft University of Technology \& Royal Haskoning

ISBN 978-90-365-4014-8

DOI: $10.3990 / 1.9789036540148$

URL: http://dx.doi.org/10.3990/1.9789036540148

Copyright (c) 2015 by Wen L. Chen

Cover photo: Hurricane Wilma satellite image photo, stock photography by NOAA

Printed by Gildeprint Drukkerijen, Enschede, The Netherlands 


\section{IDEALISED MODELLING OF STORM SURGES IN LARGE-SCALE COASTAL BASINS}

\section{DISSERTATION}

to obtain

the degree of doctor at the University of Twente, on the authority of the rector magnificus,

Prof. dr. H. Brinksma, on account of the decision of the graduation committee, to be publicly defended on Wednesday 16 December, 2015 at 12.45 hrs

by

WenLong Chen

born on 12 January 1988

in JiangShan, ZheJiang, China 
This thesis has been approved by:

Prof. dr. S.J.M.H. Hulscher

Dr. ir. P.C. Roos

Dr. ir. H.M. Schuttelaars
Promotor

Co-promotor

Co-promotor 
Advice from the ocean:

Be shore of yourself

Come out of your shell

Take time to coast

Avoid pier pressure

Sea life's beauty

Don't get tide down

Make waves!

-Ilan Shamir 



\section{Contents}

$\begin{array}{ll}\text { Preface } & 12\end{array}$

$\begin{array}{ll}\text { Summary } & 14\end{array}$

$\begin{array}{ll}\text { Samenvatting } & 16\end{array}$

1 Introduction 17

1.1 Storm surges and coastal safety . . . . . . . . . . . . . 17

1.2 Atmospheric forcing . . . . . . . . . . . . . . . . 17

1.2 .1 Wind . . . . . . . . . . . . . . . 17

1.2 .2 Low pressure system . . . . . . . . . . . . . . . . . . 19

1.2 .3 Tropical storms . . . . . . . . . . . . . . . . . . . 21

1.3 Hydrodynamic response . . . . . . . . . . . . . . . . . 22

1.3.1 Wind driven set-up in elongated closed basins . . . . . . . 22

1.3.2 Influence of topography, Coriolis effect and spatial pattern

of wind field . . . . . . . . . . . . . . . . 25

1.3 .3 Low pressure effect . . . . . . . . . . . . . . 26

1.3.4 Storm surges . . . . . . . . . . . . . . . . 27

1.3.5 Overview of model approaches . . . . . . . . . . . . 28

1.3.6 Knowledge gaps . . . . . . . . . . . . . . . . . . . . 29

1.4 Research contents . . . . . . . . . . . . . . . . . . . . . . 29

1.4.1 Research goal . . . . . . . . . . . . . . . . . . . . 29

1.4 .2 Research questions . . . . . . . . . . . . . . . . . . . . 29

1.4.3 Research Methodology . . . . . . . . . . . . . . . . . . . . 29

1.4.4 Outline of the thesis . . . . . . . . . . . . . . 30

1.A Details of the simple wind-driven flow model . . . . . . . . 32

1.A.1 Dynamic equilibrium solution . . . . . . . . . . . . . 32

1.A.2 Transient solution for pure resonance . . . . . . . . . 32

1.A.3 Energy argument . . . . . . . . . . . . . . . . . . 33

2 Resonance properties of a closed rotating rectangular basin subject to space- and time-dependent wind forcing 35

2.1 Introduction . . . . . . . . . . . . . . . . . . . . 35

2.2 Model formulation . . . . . . . . . . . . . . . . . . . 37

2.3 Solution method . . . . . . . . . . . . . . . . . 39

2.3.1 Differential problem for surface elevation amplitude . . . 39

2.3 .2 Collocation method . . . . . . . . . . . . . . . 40 
2.3.3 Analytical solution for small $f / \omega$ and free slip . . . . . 41

2.4 Results . . . . . . . . . . . . . . . . . . 42

2.4.1 Collocation method . . . . . . . . . . . . . . . 42

2.4.2 Analytical solution for small $f / \omega$ and free slip . . . . . 46

2.5 Discussion . . . . . . . . . . . . . . . . . . 47

2.5.1 Interpretation of the resonances . . . . . . . . . . . 47

2.5.2 Influence of basin dimensions . . . . . . . . . . . . . 50

2.5.3 Single wind event . . . . . . . . . . . . . . . . 50

2.6 Conclusions . . . . . . . . . . . . . . . . . 51

2.A Expressions for flow and problem for $N \ldots \ldots . . . . .53$

2.A.1 Vertical profiles from horizontal momentum equations . . 53

2.A.2 Elliptical problem for $N$. . . . . . . . . . . . . 54

2.A.3 Vertical flow velocity . . . . . . . . . . . . . . 54

2.B Details of the collocation method . . . . . . . . . . . . . 54

2.B.1 Expressions for $\phi^{\text {unif }}, \phi^{\text {div }}$ and $\phi^{\text {curl }} \ldots . . . . . . . .554$

2.B.2 Convergence test for spatially uniform wind . . . . . . . 55

2.B.3 Channel modes: Kelvin and Poincaré waves . . . . . . . . 55

2.B.4 Equilibrium response to steady wind forcing $(\omega=0)$. . . 56

2.C Details of the expansion in $f / \omega \ldots \ldots . \ldots 57$

3 Response of large-scale coastal basins to wind forcing: influence $\begin{array}{ll}\text { of topography } & 59\end{array}$

3.1 Introduction . . . . . . . . . . . . . . . . . . . . 59

3.2 Model formulation . . . . . . . . . . . . . . . . . 61

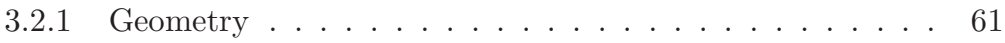

3.2.2 Hydrodynamics . . . . . . . . . . . . . . . . 62

3.3 Solution method . . . . . . . . . . . . . . . 64

3.3.1 Preliminary considerations: wind angle . . . . . . . . 64

3.3.2 Differential problem for surface elevation amplitude . . . 65

3.3.3 Finite Element Method . . . . . . . . . . . . 65

3.4 Results and discussion . . . . . . . . . . . . . . . . . . . . 67

3.4 .1 Introduction . . . . . . . . . . . . . . 67

3.4.2 Uniform depth; influence of wind direction . . . . . . . . . 68

3.4.3 Abrupt topographic step . . . . . . . . . . . . 70

3.4.4 Influence of slope length (smoothened step) . . . . . . . . 74

3.4.5 Linear profile . . . . . . . . . . . . . . . . . 75

3.4.6 Parabolic cross-basin profile . . . . . . . . . . . 76

3.5 Conclusions . . . . . . . . . . . . . . . . . . . 78

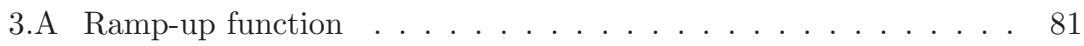

3.B Details of the derivation . . . . . . . . . . . . . 81 3.B.1 Vertical profiles from horizontal momentum equations . . 81

3.B.2 Elliptical problem for $N \ldots \ldots$. . . . . . . . . 82

3.B.3 Vertical flow velocity . . . . . . . . . . . . . . . . 82

3.C Collocation method . . . . . . . . . . . . . . . . . 82

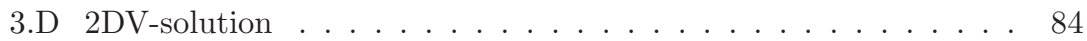

3.E Wave modes . . . . . . . . . . . . . . . . . 85 
4 The influence of storm characteristics on storm surge $\quad 87$

4.1 Introduction . . . . . . . . . . . . . . . . . . 87

4.2 Model formulation . . . . . . . . . . . . . . . . . . . . . 90

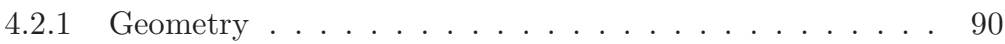

4.2.2 Hydrodynamics . . . . . . . . . . . . . . . . . . . . . . . . . . . . . . 90

4.2.3 Atmospheric forcing . . . . . . . . . . . . . . . . . 91

4.3 Solution method . . . . . . . . . . . . . . . . . 92

4.3.1 Fourier expansion of the problem . . . . . . . . . . 92

4.3.2 Differential problem for surface elevation amplitude at

each frequency . . . . . . . . . . . . . . . . . . 94

4.3.3 Finite Element Method . . . . . . . . . . . . . 95

4.4 Modelling the Hurricane Katrina surge . . . . . . . . . . . . . . . 95

4.4.1 Geometry and bathymetry . . . . . . . . . . . . 95

4.4.2 Derivation of storm parameters . . . . . . . . . . . . 97

4.4.3 Hydrodynamic parameters and numerical setting . . . . . 100

4.4 .4 Model results . . . . . . . . . . . . . . . . . 100

4.5 Sensitivity of surge levels to storm characteristics . . . . . . . . . 101

4.5.1 Synthetic storms . . . . . . . . . . . . . . . 101

4.5.2 Sensitivity to storm direction . . . . . . . . . . . . 102

4.5.3 Sensitivity to landfall point . . . . . . . . . . . . . . 104

4.5.4 Sensitivity to storm size . . . . . . . . . . . 106

4.5.5 Sensitivity to forward speed . . . . . . . . . . . . 107

4.5.6 Sensitivity to central pressure . . . . . . . . . . . 108

4.5.7 Forerunner .................. . . 110

4.5.8 Fourier spectrum of the elevation amplitude . . . . . . . 110

4.6 Discussion and conclusion . . . . . . . . . . . . . . . . . 112

4.A Details of the derivation . . . . . . . . . . . . . . . . . 114

4.A.1 Vertical profiles from horizontal momentum equations . . 114

4.A.2 Elliptical problem for $N$. . . . . . . . . . . . 115

4.A.3 Vertical flow velocity . . . . . . . . . . . . . . 115

4.B Convergence test for Hurricane Katrina surge . . . . . . . . . . 116

4.C Fourier representation of atmospheric forcing . . . . . . . . . 116

5 Discussion, conclusions and recommendations $\quad 119$

5.1 Discussion . . . . . . . . . . . . . . . . . . . 119

5.2 Answers to the research questions . . . . . . . . . . . . . 120

5.3 Overall conclusions . . . . . . . . . . . . . . . . . . . . 122

5.4 Recommendations .................... 122

$\begin{array}{lr}\text { Bibliography } & \mathbf{1 3 0}\end{array}$

$\begin{array}{lr}\text { List of publications } & 131\end{array}$

$\begin{array}{ll}\text { About the author } & 133\end{array}$ 


\section{Preface}

My Ph.D. adventure is a long journey, in the sense of distance (China-Netherlands), time (2011-2015) and differs in culture (hot water-cappuccino). During this trip, I have encountered a number of brilliant people who brought joy and passion to me along my adventure. Without their contribution, this thesis would not be possible. Therefore, I would like to express my gratitude to the following people.

First of all, I'd like to thank Suzanne Hulscher for accepting me as a Ph.D. candidate. I appreciate your enthusiastic supports, critical suggestions, availability for discussions and kindness towards me, which ensures the success of this project. I sincerely appreciate your mentorship.

I am very grateful to my daily supervisor, Pieter Roos, for bringing me to this project, teaching me the scientific way of thinking and correcting every tiny error in my code and report. Words are not enough to express my gratitude to you, Pieter. This project would not be possible without your help. Your enthusiasm, endless patience for discussion and proofreading, pursuit for perfection and sense of humour (key), made me enjoy every second of our cooperation.

Next, I would like to thank Henk Schuttelaars for your guidance. You have guided me through this project together with Pieter and Suzanne. Your sharp and critical comments ensure the scientific quality of this thesis and your ability to simplify difficult problems clears the cloud of this project. Because of you and Pieter, this project is full of fasinating physics, elegant mathematics and funny jokes (although sometimes it's difficult for me to follow).

A special thank goes to Huib de Swart, Mohit Kumar, Mathijs Van Ledden and Tjerk Zitman. Huib for his interest in this project, organizing reading group and partly funding this project; Mohit for his valuable contributions to the numerical modelling part of this project; Mathijs for bringing me to the Hurricane Katrina case, his help in finding sources and discussing model results; and Tjerk for his nice suggestion on the model and humorous meetings. Leonie Straatsma is gratefully acknowledged for her contributions to the surge sensitivity study. I extend my appreciation to Jan Mulder, Wiebe De Boer, Ad stolk and Floris Groenendijk for being members of user group of this project. They have brought real-life issues into this project with constructive and practical suggestions.

I would like to express my gratitude to Chinese Scholarship Council (CSC) and the research programme 'Impact of climate change and human intervention on hydrodynamics and environmental conditions in the Ems-Dollart estuary: an integrated data-modelling approach' for the financial support to this project. The latter programme is financed by the Bundesministerium fur Bil- 
dung und Forschung (BMBF) and by the Netherlands Organization for Scientific Research (NWO), as part of the international Wadden Sea programme (GEORISK project).

Furthermore, I want to thank my colleagues of Water Engineering \& Management Group, particularly to Abebe Chukalla and Leonardo Duarte Campos for being my paranymph and being ready for questions during the defense. My appreciation also goes to Wouter Kranenburg, Abebe Chukalla, John Damen and Isaac Williams, with whom I have had the pleasure to share the office. In this most international office, we have shared so many nice memories, such as the Chinese/Ethiopia/Netherlands corner, discussions on science and religion, coffee breaks and gezondheid when sneezing! There are many more colleagues I would like to thank for making my enjoyable stay at the WEM department. I thank them for arranging WEM-'uitjes' activity, organising daghap dinner, joining in the BATA-race, brining delicious food (Colombia, Chile, Chinese, Indonesia, Dutch, Iran, Spain etc), sharing lunch walk and of course participating in NCK summer school. I also want to thank the secretaries Anke WiggerGroothuijs, Joke Meijer-Lentelink and Monique te Vaarwerk (earlier Brigitte Leurink) for providing a pleasant working atmosphere.

Besides the colleagues, I would like to thank my friends outside the group, especially those fellows from my motherland. I thank them for sharing parties, dinners, sports (swim, basketball, snooker and table tennis), games (board game and MaJiang) and travellings with me. Special thanks go to Junwen Luo and Liang Ye, for their friendship and for cheering me up when I was upset.

Finally, I am very grateful to my family. Without their unconditional support, this thesis would not have been here. I would like to express my affection to Yucong Gao. Your love and support are the tower light which guides me through the darkness of storms.

Wenlong Chen

Enschede, November 2015 


\section{Summary}

Coastal areas around the world are frequently attacked by various types of storms, threatening human life and property. This study aims to understand storm surge processes in large-scale coastal basins, particularly focusing on the influences of geometry, topography and storm characteristics on the water levels along the coast. To this end, an idealised process-based hydrodynamic model is developed. For arbitrary closed or semi-enclosed basins, it solves the linearised three-dimensional shallow water equations, including the Coriolis effect, forced by time- and space-dependent wind and pressure fields. The model is linear which allows us to analyse the problem in terms of the basin's frequency response (which reflects its resonance properties). The model is fast because it decouples the vertical calculations, which are analytical, from the horizontal calculations, which are carried out using the Finite Element Method (or, in simplified cases, using semi-analytical techniques).

The spectral response of a closed rectangular basin of uniform depth, subject to periodic wind forcing, is studied first (Chapter 2). It is found to depend on the spatial characteristics of the wind field and the basin dimensions. In particular, it is shown that different spatial wind patterns (uniform wind, wind with nonzero divergence and wind with nonzero curl) produce different resonance peaks. The response is further modified by bottom friction, lowering the resonance peaks, and the Coriolis effect. The latter causes the peaks to shift, and new peaks to emerge associated with cross-wind basin dynamics.

Then, the influence of topographic elements on the spectral response is studied (Chapter 3), in semi-enclosed basins. The results point out that adding topographic elements (such as a topographic step, a linearly sloping bed or a parabolic cross-basin profile) causes the resonance peaks to shift in the frequency domain, through their effect on local wave speed.

The influence of storm characteristics on the set-up (or set-down) along the coast in the New Orleans coastal basin is investigated in Chapter 4. First, it is shown that the model, with a schematised domain and forced by the so-called Holland-B model, is able to qualitatively reproduce the surge produced by Hurricane Katrina (2005). A sensitivity study is carried out in which the storm parameters are varied around the values that are representative for Hurricane Katrina. The storm direction and point of landfall are found to be the most important parameters determining the surge height. In particular, a storm approaching from south-east making landfall at the seaward end of the Mississippi dike produces the highest surge levels.

Due to its flexibility regarding geometry, topography and forcing, the new 
idealised model can be applied to other locations than the New Orleans coastal basin. The short calculation times make it a quick estimation tool for extensive sensitivity studies. With these properties, the new model bridges the gap between the more theoretical non-site-specific model studies and the computationally expensive detailed site-specific numerical simulation models. 


\section{Samenvatting}

Stormen in kustgebieden vormen een gevaar voor mensenlevens en hun bezittingen. Het doel van deze studie is om een beter begrip te krijgen van stormvloeden in grootschalige bekkens, en dan met name wat betreft de specifieke invloeden van geometrie, bodemligging en stormeigenschappen op de waterstanden langs de kust. Hiertoe wordt een gedealiseerd proces-gebaseerd model ontwikkeld. Voor een willekeurig gesloten of half-ingesloten bekken worden de 3D ondiepwatervergelijkingen opgelost, inclusief het Coriolis-effect, geforceerd door winden drukvelden die variren in ruimte en tijd. Omdat het model lineair is, kan het probleem worden geanalyseerd in termen van de zogeheten spectrale respons. Hierin zitten de resonantie-eigenschappen van het systeem opgesloten. Het model is verder snel omdat de (analytische) verticale berekeningen ontkoppeld zijn van de horizontale berekeningen, die met een eindige-elementen-methode worden uitgevoerd (of, in enkele gevallen, met semi-analytische technieken).

Allereerst wordt de spectrale respons van een gesloten bekken met uniforme diepte onderzocht (Hoofdstuk 2). Deze respons hangt af van de ruimtelijke eigenschappen van het windveld alsmede de afmetingen van het bekken. In het bijzonder wordt duidelijk hoe verschillende ruimtelijke patronen (uniform windveld, wind met divergentie, wind met rotatie) tot verschillende resonantiepieken leiden. De response wordt verder benvloed door bodemwrijving, die de pieken dempt, en door het Coriolis effect, dat de pieken doet verschuiven (en nieuwe pieken doet ontstaan die te maken hebben met dynamica in de richting loodrecht op de wind).

Vervolgens wordt de invloed van topografische elementen onderzocht (Hoofdstuk 3), voor half-ingesloten bekkens. De modelresultaten laten zien hoe dergelijke elementen (zoals een diepte-stap, een bodemhelling of een parabolisch dwarsprofiel) de resonantiepieken doen verschuiven, door hun effect of op de lokale golfvoortplantingssnelheid.

De invloed van stormeigenschappen op de op- en afwaaiing langs de kust van het New Orleans bekken wordt onderzocht in hoofdstuk 4. Eerst wordt getoond dat het model, met een geschematiseerde domein en geforceerd door het zogeheten Holland-B model, in staat is om kwalitatief de stormvloed van Orkaan Katrina (2005) te reproduceren. Een gevoeligheidsstudie laat vervolgens zien dat de richting van de stormkoers en het punt waar de orkaan aan land komt de belangrijkste stormparameters zijn. In het bijzonder blijkt dat de hoogste waterstanden worden gevonden voor een storm vanuit uit het zuidoosten die aan land gaat aan de zeezijde van de Mississippi Dike.

Vanwege haar flexibiliteit kan het model ook op andere locaties worden 
toegepast. De korte rekentijden maken het model geschikt voor uitgebreide gevoeligheidsstudies. Dit overbrugt de kloof tussen de theoretische, generieke studies en rekenintensieve numerieke simulatiemodellen. 


\section{Chapter 1}

\section{Introduction}

\subsection{Storm surges and coastal safety}

Each year, many storms attack coastal areas. These storms pose a major safety threat to coastal environments which are often densely populated areas. Indeed, high surge levels along the coast can cause coastal inundations which will damage roads and bridges and destroy homes and businesses. The rising sea level resulting from global warming further increase the vulnerability of the coastal region to storm surges.

There is a large variability in storms and storm surges. However, it is unclear how surge levels and surge level distribution along the coast can be determined with these characteristics. Therefore, to protect life and properties from these damages, an overall practical goal is to be able to predict storm surge levels at any location. To achieve this goal, it is essential to improve our understanding of the storm surge processes and the factors influencing storm surges.

This thesis focuses on the understanding of storm surge processes in largescale coastal basins, particularly on the influence of geometry, topography and storm characteristics on basin response. As background information, $\S 1.2$ gives definitions and a brief description of atmospheric forcings that are involved in storms. Subsequently, $§ 1.3$ summarizes the state-of-the-art knowledge on the response of coastal basins to these forcings. The research goal and approach central to this thesis are presented in $\S 1.4$, which also contains an outline of the thesis.

\subsection{Atmospheric forcing}

Atmospheric forcings such as wind and low pressure systems are the main drivers of the water motion in storm conditions. This section introduces wind and low pressure systems. As an example of the combined effect of these two forcings, tropical storms are described.

\subsubsection{Wind}

Wind is the large-scale flow of air, caused by spatial gradients in atmospheric pressure and influenced by the earth's rotation. The highest wind speed ever recorded was during the passage of Tropical Cyclone Olivia on 10 April 1996: an 
(a) spatially uniform

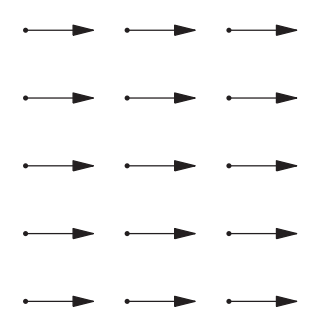

(b) nonzero divergence

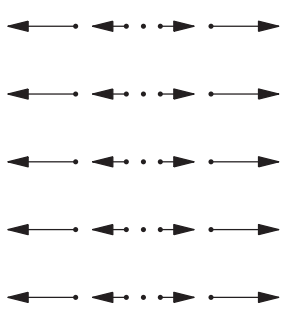

(c) nonzero curl

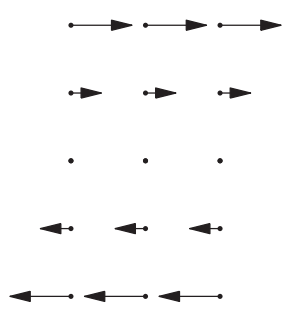

Figure 1.1: Top view of the examples of three schematised spatial patterns of wind field: $(a)$ a spatially uniform pattern, $(b)$ a wind pattern with linear variation in the along-wind direction, $(c)$ a wind pattern with linear variation in the cross-wind direction.

automatic weather station on Barrow Island, Australia, recorded a maximum wind gust of $113 \mathrm{~m} \mathrm{~s}^{-1}$ (Callaghan, 1997).

Usually, the spatial pattern of a wind field is highly variable. This applies to both the speed and direction of the wind. To facilitate the analysis, three schematised spatial patterns of wind field are usually distinguished (e.g., Mohammed-Zaki, 1980; Csanady, 1982), see figure 1.1:

- a spatially uniform pattern where wind speed and wind direction are uniform,

- a wind pattern with variation in the along-wind direction, i.e. with a nonzero divergence of the wind field,

- a wind pattern with variation in the cross-wind direction, i.e. with a nonzero curl of the wind field.

A wind field with a complex spatial pattern can be viewed as the superposition of the above three spatial patterns.

Wind fields usually also show a complicated temporal structure. In principle, it is a continuous signal with many fluctuations in both speed and direction. However, it can be simplified into three temporal patterns.

- A steady wind, in which the wind speed and direction are both constant in time.

- A single wind event, which is characterised by absence of wind, followed by a spin-up, a period with constant wind and a spin-down stage back to no wind conditions. For example, Typhoon Haiyan in Southeast Asia formed on November 3, 2013 and dissipated on November 11, 2013 (Lum and Margesoon, 2014).

- A periodic wind, of which the wind speed and direction evolves periodically. A typical example of such a periodic wind is provided by the sea 


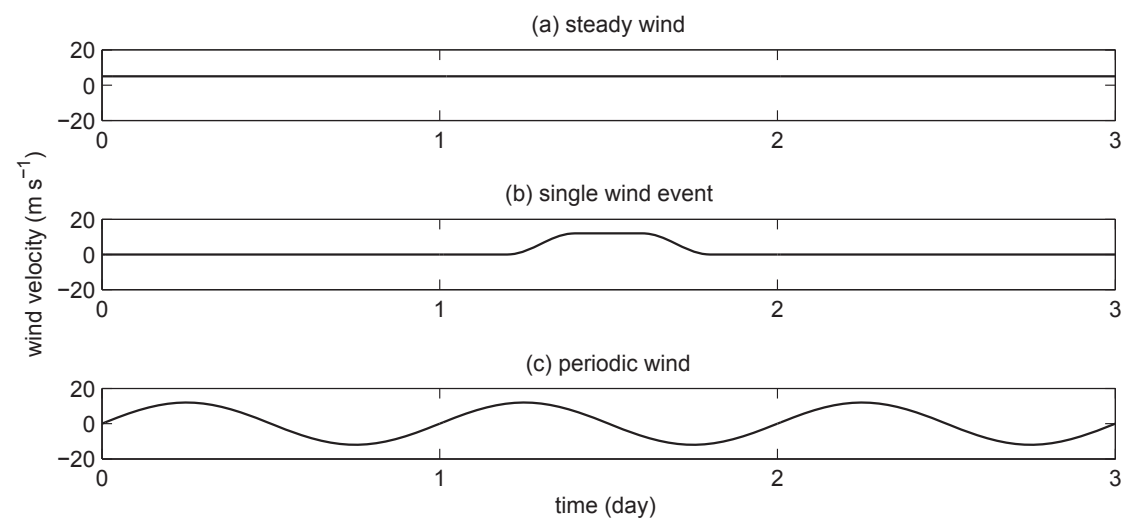

Figure 1.2: Examples of wind patterns showing wind velocity as a function of time for: $(a)$ steady wind, $(b)$ a single wind event and $(c)$ periodic wind.

and land breezes varying in a daily cycle. During the day, a sea breeze blows from the sea towards land, while at night, a land breeze blows from the land to the sea (Borne, 1998).

Figure 1.2 show examples of these three temporal patterns. It is important to emphasize that any single wind event can be written as a superposition of sinusoidal periodic wind forcings at various frequencies (Craig, 1989). This property, which connects the second and third temporal patterns described above, facilitates model studies based on linearised shallow water equations. The effect of a single wind event is then contained in the so-called frequency response of a basin to periodic wind forcings over a wide range of frequencies.

Due to the resistance of the water surface, wind blowing over the sea surface forms a bottom boundary layer in the atmosphere. The horizontal force of wind per surface area that acts on the sea surface is called the wind stress $\boldsymbol{\tau}_{\mathrm{w}}=\left(\tau_{\mathrm{w}}^{(x)}, \tau_{\mathrm{w}}^{(y)}\right)$, with components in two horizontal directions. The magnitude of this stress is estimated through various wind-shear formulas (Smith et al, 1992), e.g.

$$
\boldsymbol{\tau}_{\mathrm{w}}=C_{d} \rho_{\text {air }}\left|\boldsymbol{v}_{\mathrm{w}}\right| \boldsymbol{v}_{\mathrm{w}}
$$

where $C_{\mathrm{d}}$ is the dimensionless drag coefficient with a value of the order of $10^{-3}$ (Garratt, 1977; Wu, 1980; Resio and Westerink, 2008). Moreover, $\rho_{\text {air }}$ is the air density, which has a value of approximately $1.225 \mathrm{~kg} \mathrm{~m}^{-3}$. The vector $\boldsymbol{v}_{\mathrm{w}}$ denotes the two-dimensional horizontal wind velocity vector evaluated at $10 \mathrm{~m}$ above the sea surface.

\subsubsection{Low pressure system}

The air pressure is not uniform over the earth surface. These differences are caused by unequal heating together with the earth's gravitational force. A low 


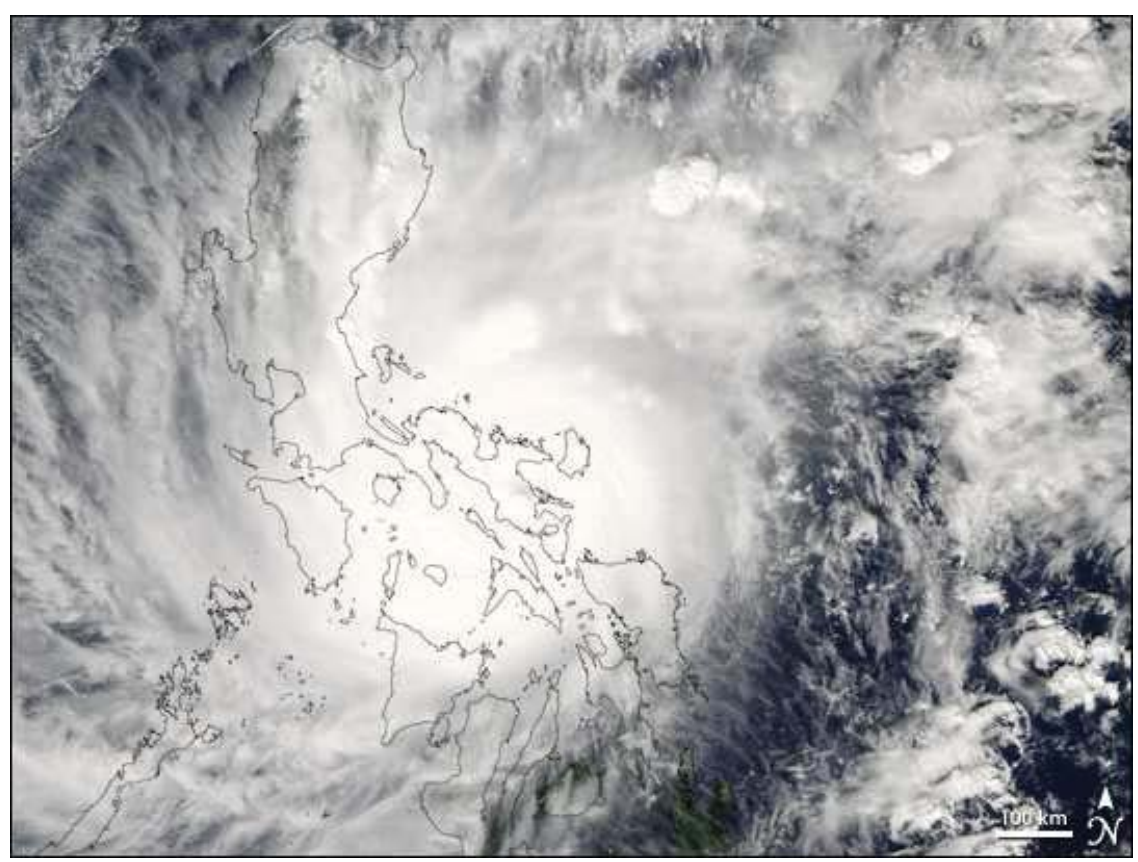

Figure 1.3: Satellite image of super Typhoon Durian which crossed the Philippines on November 30, 2006 (National Aeronautics and Space Administration, http://earthobservatory.nasa.gov/NaturalHazards/view.php?id=17695).

pressure system is an area where the atmospheric pressure is lower than that of the surrounding area.

A low pressure system is often accompanied by strong winds. At mid-latitude (between $30^{\circ}$ and $60^{\circ}$ ), it may develop into an extratropical storm. Alternatively, a low pressure system over tropical or subtropical waters can develop into a tropical storm. Extratropical and tropical storms differ in their way of obtaining energy. An extratropical storm gains energy from the release of potential energy when cold and warm air masses interact. On the other hand, a tropical storm obtains energy from latent heat which is released when water vapor condenses into liquid water (Abbott, 1996). The difference is important, since tropical storms have the potential to quickly grow into hurricanes, whereas extratropical storms do not. Tropical storms and hurricanes may cause huge damages to life and properties. According to Hough (2008), hurricanes account for 3 out of 10 of the worst natural disasters. Moreover, compared to an extratropical storm, the wind and pressure field structures of a tropical storm are simpler and are specified using parametric models such as that by Holland (1980). As part of this study focuses on tropical storms, a brief introduction on tropical storms is given below. 
(a) hurricane

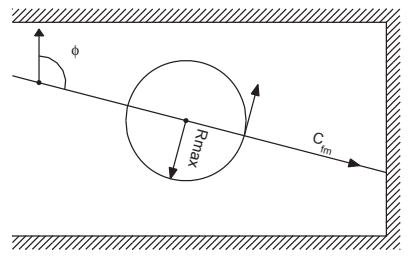

(b) pressure and wind field

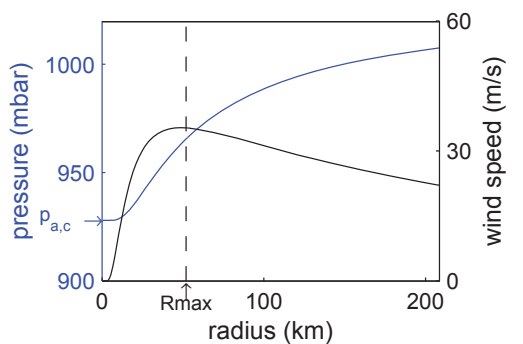

Figure 1.4: Top view sketch of a storm moving over a coastal basin, showing a: $(a)$ storm track, with $\phi$ denoting the storm direction, $C_{\mathrm{fm}}$ the forward speed of storm and $R_{\max }$ the distance to maximum wind speed, $(b)$ pressure (blue curve) and wind speed (black curve) as function of the dimensionless distance away from storm centre, the black dashed line indicates the maximum radius $R_{\max }$ and the blue arrow refers to the central pressure $p_{a, c}$.

\subsubsection{Tropical storms}

A tropical storm is defined as a warm-core, low-pressure system without any front attached to it, which develops over the tropical or subtropical waters, and has an organised circulation (Murck et al, 1997). Depending upon location, it is called 'typhoon' (literally, 'great wind') in the northwest Pacific, 'hurricane' in the northwest Atlantic (of Caribbean origin) and northeast Pacific, or 'tropical cyclone' in tropical areas of the southwest Pacific and of the Indian Ocean (James, 1998). Figure 1.3 shows an example of a tropical storm.

The low pressure system may first grow into a tropical depression, of which the maximum sustained wind velocity is defined as $17 \mathrm{~m} \mathrm{~s}^{-1}$. With sufficient energy input, it will further develop into a tropical storm, or even a hurricane.

At the centre of the storm, there is a calm region of roughly circular shape where the pressure is minimum, known as the 'eye' of the storm. Atmospheric pressure increases roughly exponentially when moving away from the storm's eye. This pressure gradient forces a wind blowing from the surrounding high pressure area to the low pressure area at the centre. Due to the Coriolis force, it circulates around the centre in a cyclonic fashion, i.e. counterclockwise (clockwise) in the Northern (Southern) Hemisphere. Importantly, the wind speed in the centre is zero. The wind speed first increases away from the centre until it reaches a maximum value at some radius $R_{\max }$, whereas further away from the eye it gradually decreases again.

Despite the complexity of the atmospheric forcings in space and time, the main storm features are captured by a relatively small set of parameters. For example, in his so-called Holland-B model, Holland (1980) used six parameters to describe the radial profile of pressure and wind in a storm:

Central pressure $\left(p_{\mathrm{a}, \mathrm{c}}\right)$ is an indicator of the storm intensity. The central pressure can be as low as 900 mbar, as recorded when Hurricane Camille 
made landfall in New Orleans in 1969 (Corps of Engineers, 1970).

Distance to maximum wind speed $\left(R_{\max }\right)$, measured from the storm's centre, indicates the size of the storm. A small storm such as Hurricane Dennis in the Gulf of Mexico (August 2005) had a radius of $11 \mathrm{~km}$, while Hurricane Wilma showed a radius of $73 \mathrm{~km}$ (Blake et al, 2006).

Forward speed of storm $\left(C_{\mathrm{fm}}\right)$ is the speed at which the storm centre moves. For example, Hurricane Sandy moved at a speed of $12.5 \mathrm{~m} \mathrm{~s}^{-1}$ at the time of landfall (Blake et al, 2013).

Storm location $(x, y)$ and storm direction $\phi$ define the track of a storm, indicating the initial location of the storm's centre and the direction of storm motion, respectively. Taking a constant $\phi$ value would define a straight line, although in reality a storm usually changes direction as it approaches land.

Holland-B parameter describes the peakedness of the pressure profile relative to the storm centre. A higher value results in a steeper slope of the pressure profile.

Figure 1.4 shows an example of a storm track together with the pressure and wind field of a storm calculated from the Holland-B model. The model is widely used for its simplicity and flexibility in radial structure (Madsen and Jakobsen, 2004).

\subsection{Hydrodynamic response}

When the atmospheric forcings work on coastal waters, water is set into motion which results in set-up or set-down at the coasts. In this section, the wind driven set-up in elongated closed basins is first presented using a simple model (§1.3.1). Then, complications on the wind-driven flow introduced by topography, Coriolis effect and the spatial pattern of the wind field are discussed ( $\S 1.3 .2)$. Next, $\S 1.3 .3$ discusses the effect of a low pressure system followed by a review of recent studies on the combined effect of wind and low pressure systems on storm surges (§1.3.4). Finally, $\S 1.3 .5$ presents an overview of model approaches to model the hydrodynamic response and $\S 1.3 .6$ summarises the knowledge gaps.

\subsubsection{Wind driven set-up in elongated closed basins}

In this subsection, we introduce a simple one-dimensional model of wind-driven flow in a closed basin. Our aim is to illustrate two aspects: (i) the equilibrium response to a steady wind, and (ii) the possibly resonant response to an oscillatory wind. To this end, let us consider depth-averaged flow $\bar{u}(x, t)$ in a shallow elongated basin of length $L$ and uniform depth $h$. Assuming the surface elevation $\eta(x, t)$ to be small compared to the water depth, the unknowns $\eta$ and $\bar{u}$ satisfy the linearised shallow water equations:

$$
\frac{\partial \bar{u}}{\partial t}+\frac{r \bar{u}}{h}=-g \frac{\partial \eta}{\partial x}+\frac{\tau_{\mathrm{w}}^{(x)}}{\rho h}, \quad \frac{\partial \eta}{\partial t}+h \frac{\partial \bar{u}}{\partial x}=0 .
$$




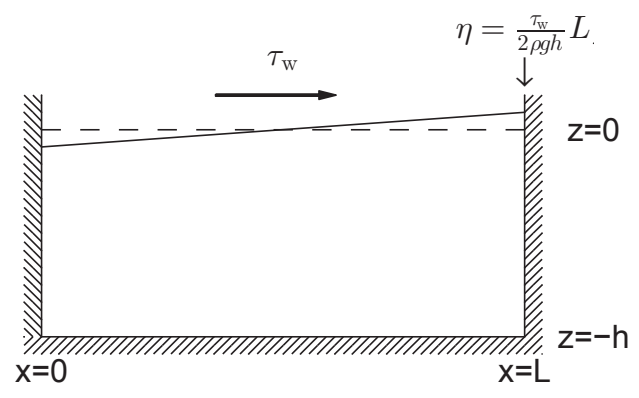

Figure 1.5: Wind driven set-up in an elongated closed basin

Here, $r$ is a linear bottom friction coefficient resulting from Lorentz' linearization (Lorentz, 1922), $g$ the gravitational acceleration, $\tau_{\mathrm{w}}^{(x)}$ the wind stress, considered spatially uniform, and $\rho$ the water density. Closed boundary conditions require $\bar{u}(0, t)=\bar{u}(L, t)=0$. Initially, the basin is at rest: $\eta(x, 0)=0$ and $\bar{u}(x, 0)=$ 0 . Assuming a steady wind stress in the positive $x$-direction $\left(\tau_{\mathrm{w}}^{(x)}>0\right)$, the equilibrium response surface profile satisfies

$$
\frac{\partial \eta}{\partial x}=\frac{\tau_{\mathrm{w}}^{(x)}}{\rho g h}
$$

implying a linear surface profile with set-up $\eta(L)=\tau_{\mathrm{w}}^{(x)} L /(2 \rho g h)$ at the downwind boundary, see Fig.1.5.

Alternatively, we may consider a time-periodic wind stress $\tau_{\mathrm{w}}^{(x)} / \rho=\hat{T} \sin \omega t$ with amplitude $\hat{T}$ and angular frequency $\omega$. Neglecting bottom friction $(r=0)$, the dynamic equilibrium response is then given by

$$
\eta(x, t)=\frac{\hat{T}}{g h k}\left[\sin k x-\left(\frac{1-\cos k L}{\sin k L}\right) \cos k x\right] \sin \omega t,
$$

with shallow water wave number $k=\omega / \sqrt{g h}$. This result clearly shows that the response depends on the forcing frequency. In particular, large amplification occurs when $k L \approx \tilde{n} \pi$ for some odd $\tilde{n}$, i.e. when the basin length is close to an odd multiple of half the shallow water wavelength $\frac{1}{2} \lambda=\pi / k$. If it exactly equals an odd multiple of $\frac{1}{2} \lambda$, a singularity occurs in Eq.(1.4). The assumption of a time-periodic dynamic equilibrium solution then breaks down and we should turn to the transient problem (starting from rest).

As shown in Appendix 1.A, the transient solution is a superposition of oscillatory modes, one of which has an amplitude that increases linearly with time:

$$
\eta(x, t)=\underbrace{A_{\tilde{n}} \omega t \cos \left(\frac{\tilde{n} \pi x}{L}\right) \cos \omega t}_{\text {see Figure } 1.6}+\text { other terms, }
$$


(a) surface elevation at right boundary $(x=L)$

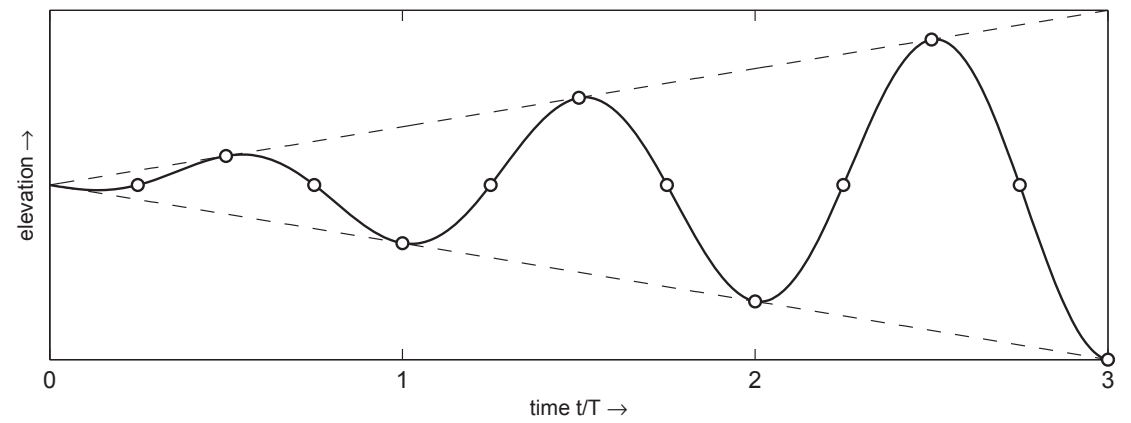

(b) spatial patterns of wind stress, surface elevation and flow

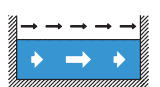

$\mathrm{t} / \mathrm{T}=0.25$

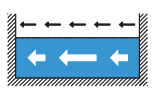

$\mathrm{t} / \mathrm{T}=1.75$

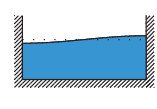

$t / T=0.5$

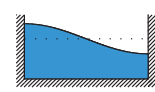

$\mathrm{t} / \mathrm{T}=2$

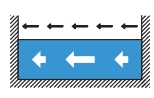

$\mathrm{t} / \mathrm{T}=0.75$

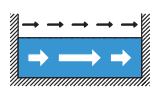

$\mathrm{t} / \mathrm{T}=2.25$

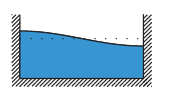

$t / T=1$

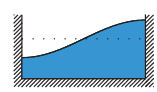

$\mathrm{t} / \mathrm{T}=2.5$

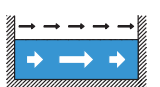

$\mathrm{t} / \mathrm{T}=1.25$

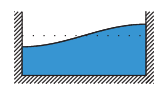

$\mathrm{t} / \mathrm{T}=1.5$

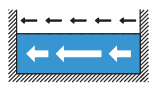

$\mathrm{t} / \mathrm{T}=2.75$

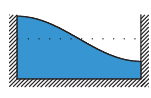

$t / T=3$

Figure 1.6: Example of pure resonance in an elongated basin subject to time-periodic wind with a period $T=2 \pi / \omega$. (a) Time evolution of the surface elevation at the righthand boundary $(x=L)$ of the $\tilde{n}$-th mode, which shows an amplitude that increases linearly with time (dashed lines). (b) Spatial patterns of wind stress (black arrows), surface elevation and depth-averaged flow (white arrows), evaluated at the moments indicated by circles in the top panel. This figure, in which $\tilde{n}=1$, only depicts the dynamics associated with the underbraced term in Eq.(1.5).

with coefficient $A_{\tilde{n}}$ specified in Appendix 1.A.2 (along with the 'other terms'). Equation (1.5) provides an example of pure resonance. From a physical perspective, there is a continuous transfer of wind power onto the $\tilde{n}$-th mode of the system (see Appendix 1.A.3). This is illustrated in Fig.1.6, which shows the time evolution of the surface elevation at the right-hand boundary and the spatial patterns of wind stress, surface elevation and depth-averaged flow of the $\tilde{n}$-th mode with $\tilde{n}=1$.

We conclude that the singularities in the dynamic equilibrium solution in Eq.(1.4) indicate conditions of pure resonance. The presence of bottom friction $(r>0)$ excludes the possibility of pure resonance, but still allows for large amplification peaks. This situation is termed practical resonance, which expresses a balance between the power input due to the wind stress acting on the water surface and the dissipation due to bottom friction. 


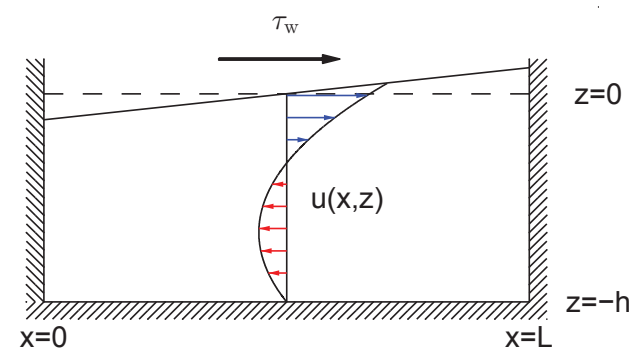

Figure 1.7: Wind driven flow and set-up in an elongated closed basin, side view and a sketch of velocity profile, blue and red arrows indicate downwind and upwind flow, respectively.

\subsubsection{Influence of topography, Coriolis effect and spatial pattern of wind field}

In reality, vertical mixing causes the wind-driven velocity to vary with the vertical coordinate. In the absence of the veering effect introduced by the earth's rotation, the surface flow goes downwind, resulting in a pressure gradient in the presence of closed boundary. Furthermore, this pressure gradient drives a return current in the bottom layer to compensate the downwind surface flow, as shown in Figure 1.7.

This steady circulation is modified when there are variations in topography. Csanady (1968b) investigated the equilibrium wind driven water motion of an elongated coastal basin, in which the depth contours are parallel to the shores. Where the water is shallower than the average depth of the basin, transport is in the direction of the wind; it is in the opposite direction in the deeper parts. Topography also affects the time-dependent response of the basin to wind forcing. This is reflected in the effect of large-scale topographic elements on the resonance properties of coastal basins. For example, shoals may protect the coast (Hanley et al, 2014), while on the other hand, a longshore bar can generate storm wave resonance under certain circumstances (Büsching, 2003). Looking at the frequency response, Proudman (1929) provided analytical solutions for the response in narrow closed basins with a single topographic step. Alternatively, Ponte (2010) investigated the response of large-scale, elongated closed basins with a parabolic cross-basin topography to along-basin wind forcing. Other studies regarding the influence of topography on the response to a moving wind forcing are mainly site-specific (e.g., Irish et al, 2008; Libicki and Bedford, 1990). To understand the influence of large-scale topography on the resonance properties of large-scale coastal basins subject to wind forcing, a systematic investigation is necessary.

Depending on the relative importance of rotation and friction (e.g., Ekman, 1905; Csanady, 1982), earth rotation may modify the velocity field. This balance is expressed in the Ekman number $\delta_{E}=h^{-1} \sqrt{2 K / f}$ with water depth $h$, vertical eddy viscosity $K$ and Coriolis parameter $f$ which is given by $f=2 \Omega \sin \vartheta$ (with 


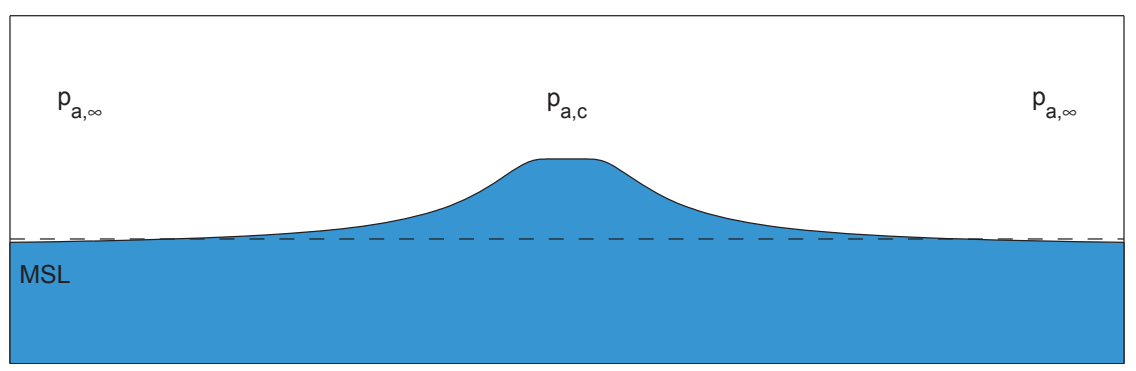

Figure 1.8: Inverted barometer effect, showing an elevated surface over low pressure areas. The black dashed line indicates the mean sea level.

$\Omega=7.292 \times 10^{-5} \mathrm{rad} \mathrm{s}^{-1}$ the angular frequency of the earth's rotation and $\vartheta$ the latitude). Focusing on the circulation in closed basins, in shallow/highly turbulent basins (small $\delta_{E}$ ) (Mathieu et al, 2002; Winant, 2004) cross-wind flows are weak, whereas they are strong in deep/weakly turbulent basins (large $\delta_{E}$ ). For a deep ocean in the Northern Hemisphere, the Coriolis effect deflects the surface currents to $45^{\circ}$ the right of the wind stress vector. The flow field spirals downward in a clockwise fashion. When integrated over depth, this deflected flow field results in a surface Ekman transport which is directed $90^{\circ}$ to the right of the wind stress. So an along-shore wind in Northern Hemisphere creates a pile-up of water when the coastline is to the right of it.

Regarding time-dependent dynamics of wind driven flow, Ponte (2010) identified a damped resonance of coastal basins subject to time-periodic wind stress when the forcing frequency is close to Coriolis frequency. Finally, the spatial variations in the wind field as observed in the Gulf of California (Ponte et al, 2012) will also affect the basin response to wind stress (e.g., Pugh, 1987; Birchfield, 1967; Mohammed-Zaki, 1980).

\subsubsection{Low pressure effect}

The sea responds to a disturbance in atmospheric pressure by adjusting its surface (Figure 1.8). This is known as the "inverted barometer effect", a terminology used by Doodson (1924). Assuming that the pressure field is static, the sea surface in equilibrium shows a steady height $\eta$ with respect to mean sea level,

$$
\eta=\frac{p_{a, \infty}-p_{a}}{\rho g},
$$

where $p_{a}$ is the atmospheric pressure, $p_{a, \infty}$ the ambient pressure, $\rho$ the sea-water density and $g$ the gravitational acceleration. This relationship is derived from the hydrostatic equation. In such an equilibrium, each millibar (mbar) drop in ambient atmospheric pressure produces roughly $1 \mathrm{~cm}$ increase in sea surface height.

Furthermore, a moving low pressure system can generate long waves (Eckart, 1951; Whitham, 1979). For example, Yankovsky (2009) demonstrated that a 
storm approaching the coast may generate large-scale edge waves. In a flatbottomed ocean, the amplification of the forced wave becomes large as the translation speed of the disturbance approaches the shallow water wave speed, this is known as Proudman resonance (Proudman, 1953). Over a linearly sloping bottom, Greenspan (1956) found similar amplification when the translation speed of a longshore travelling low pressure system is close to one of the coastally trapped edge-wave modes. Vennell (2010) showed that when a storm moving slowly across a coast with an alongshore topographic step, a subcritical resonance occurs, which generates a large reflected wave traveling along the coast. These waves are modified when the effect of earth's rotation is included (Thiebaut and Vennell, 2011).

\subsubsection{Storm surges}

When a storm moves over a coast, both the wind driven set-up and the inverted barometer effect contribute to the surge height along the coast. Notice that the set-up of the storm surge is different from the total water level amplitude observed along the coast, because the total water level amplitude is the sum of contributions from surge, tides, waves, river discharge and possible prior oscillations. For extratropical storm surges, the contributions of wind driven set-up and inverted barometer effect are in general equally important (Arthur, 1964). For tropical storm surges, the wind driven set-up is dominant since the wind speeds in tropical storms are much higher than in extratropical storms(Tannehill, 1956).

The surge level that a given storm produces at a specific location is influenced by several factors. Simpson (1974) introduced the Saffir-Simpson scale to classify the severity of a storm based on the maximum sustained wind speed. He distinguished five categories that give an indication of the expected surge levels. Despite being convenient to apply, the Saffir-Simpson scale is somehow inaccurate in forecasting storm surge levels. For example, Hurricane Katrina produced a surge that is much higher than predicted by the Saffir-Simpson scale. Irish et al (2008) suggested that the large storm size (distance to maximum wind speed) may explain the extra high surge level. This is because a storm of a larger size not only affects a larger area, but the strong winds also tend to affect this area during a longer period of time. Another important factor is the forward speed of the storm. Varying a storm's forward motion may account for variations in flooded volumes (Rego and Li, 2009). Furthermore, Weisberg and Zheng (2006) identified the storm track as another important factor concerning the resulting storm surge when a storm moves over a coastal basin. For example, in Tampa Bay, Florida, a northerly approaching storm yields a higher surge than storms from other directions.

In addition to storm characteristics, local features such as topography and geometry are important factors in determining surge height. For example, Bertin et al (2012) argued that the high surge caused by storm Xynthia in Biscay Bay was due to shelf resonance. Tomkratoke et al (2015) pointed out that the interaction process between the disturbance system and the propa- 
gating surge wave in the Gulf of Thailand may induce large positive surges. Furthermore, Irish et al (2008) found that a milder shelf slope generally leads to a higher surge. Storms making landfall on concave rather than convex coastlines produce higher surge levels (Dowdeswell and Benham, 2003).

\subsubsection{Overview of model approaches}

To accurately calculate surge levels for practical purposes, numerical models are commonly used. For example, the Sea, Lake and Overland Surges from Hurricanes (SLOSH) model which solves two-dimensional depth-averaged shallow water equations is used to investigate the storm surge risk for New York city (Lin et al, 2010). The SLOSH model applies Finite difference methods to solve the depth-averaged shallow water equations. According to Jelesnianski et al (1992), the accuracy of surge heights predicted by the model is $\pm 20 \%$ provided that the hurricane is adequatedly described. Another frequently used model is the ADvanced CIRCulation (ADCIRC) Coastal Circulation and Storm Surge Model, which applies the Finite Element Method to solve the depth-averaged shallow water equations (Bunya et al, 2010; Dietrich et al, 2012). In hindcast studies, the high-resolution ADCIRC model produces quite accurate results (Westerink et al, 2008), showing the differences with observations of less than $0.5 \mathrm{~m}$ (Dietrich et al, 2012), but it is computationally expensive (Lin et al, 2014). In real-time forecasting, the predicted winds and pressure fields are used. For example, with the wind and pressure data forecasted by the Royal Netherlands Meteorological Institute, the Dutch Storm Warning Service predict the surge levels along the Dutch coast 6 hour in advance, using the Dutch continental shelf model (Verlaan et al, 2005). This model combines simulations based on the nonlinear depth-integrated shallow water equations with a so-called Kalman filter that assimilates water level observations from tide gauges at the British and Dutch coasts. In general, the predicted surge results highly depend on the accuracy of weather forecasting models (Colle et al, 2008).

Alternatively, idealised process-based models are specifically designed to obtain insight in the relevant physical processes. Geometry, forcing and physical process are schematised, retaining only the aspects that are essential for the phenomenon under study. This leads to quick models, which allow for an extensive sensitivity analysis. Following this approach, Winant (2004) developed a three-dimensional model for wind-driven circulation in elongated basins. Other examples are the study by Birchfield (1967) on the equilibrium response to steady wind in a shallow circular basin and the study by Mohammed-Zaki (1980) on the transient response to a suddenly imposed wind stress in deep circular basins. Using a depth-averaged model, Gill (1982) showed that the surge induced by a wind field moving along an open coast takes the form of forced Kelvin waves. This simple model captures the extreme surge levels observed around the North Sea in February 1953. 


\subsubsection{Knowledge gaps}

In summary, numerical model studies are site-specific and time-consuming. On the other hand, idealized model studies strongly schematize both geometry (e.g., open coast) and forcing. For large-scale semi-enclosed basins, the influence of geometry and topography on resonance properties and hence on storm surges is not yet understood.

\subsection{Research contents}

\subsubsection{Research goal}

The goal of this thesis is:

to understand storm surge processes in large-scale coastal basins, particularly the influence of geometry and topography and storm characteristics on set-up at the coast.

Here, large-scale coastal basins are those in which the motions are significantly influenced by the earth's rotation. Moreover, geometry refers to the coastlines which may form a rectangular basin or a more complex shape. Alternatively, topography in this research refers to basin-scale features such as a topographic step across the basin thus dividing the basin into an offshore part and a coastal part. The storm characteristics refer to a spatially varying wind field and also to storm characteristics (forward speed, central pressure, maximum radius, storm direction and landfall point).

\subsubsection{Research questions}

The research goal gives rise to the following research questions.

Q1. How does the frequency response of a closed rotating basin depend on basin dimensions, the spatial structure of the wind forcing and bottom friction?

Q2. What is the influence of basin-scale topography on the frequency response of large-scale coastal basins subject to wind forcing?

Q3. What is the influence of storm characteristics on the surge response in the New Orleans coastal basin?

\subsubsection{Research Methodology}

To answer the research questions formulated in $\S 1.4 .2$, I will develop an idealised process-based model. Because of the properties outlined in $\S 1.3 .5$, this approach suits the purpose of this study best. The model solves the linearised three-dimensional shallow water equations on the $f$ plane (thus including the Coriolis effect, as required for large-scale basins). Turbulence is represented using a vertical eddy viscosity and a partial slip condition at the bed (both with parameters that are spatially uniform and constant in time). Atmospheric forcing is represented using wind stress and atmospheric pressure, which in general may vary in time and space. The solution method has the following distinctive features. 
- The linearity of the model allows us to analyse the system in terms of its frequency response. Using Fourier techniques, the forcing is expressed as a superposition of harmonic signals. By linearity, the solution is then the superposition of the individual solutions obtained at each frequency.

- At each frequency, we can decouple the vertical calculations from the horizontal calculations. This is a direct consequence of the spectral approach and the linearity of the model. The vertical calculations are analytical. Depending on the complexity of the geometry and topography, the horizontal calculations are carried out using the Finite Element Method (FEM, in the most general case) or using semi-analytical techniques (collocation method, in simplified cases).

To answer research question Q1, we will consider closed rectangular basins with uniform depth. To investigate the influence of the spatial wind pattern, we impose (time-periodic) wind stress fields that are (i) spatially uniform, (ii) of nonzero divergence, and (iii) of nonzero curl. Atmospheric pressure is neglected. Because of the simplified geometry and topography, the frequency response is obtained using a collocation method. We systematically vary the basin dimensions. Finally, to analyse the influence of the Coriolis effect, we expand the solution in powers of $f / \omega$ with Coriolis parameter $f$ and forcing frequency $\omega$.

To answer research question Q2, we will consider semi-enclosed basins. We systematically add large-scale topographic elements that are representative for real basins (such as a shallow part at the head of the basin). Particular attention is paid to the open boundary condition, for which we formulate a non-reflective condition that is valid in the presence of the Coriolis effect. Wind stress is timeperiodic and spatially uniform and is allowed to have an arbitrary orientation with respect to the along-basin direction; atmospheric pressure is neglected. Because of the more complex topography, the frequency response is now obtained using FEM.

To answer research question Q3, we consider a schematised representation of the New Orleans coastal basin. The atmospheric forcing of the model is the Holland-B model, as already introduced in $\$ 1.2 .3$, which implies time- and space-varying wind and pressure fields. The solution is obtained using FEM. To gain confidence in the model, it is first applied to simulate the surge caused by Hurricane Katrina. At a set of coastal locations, the surge levels obtained with our model are compared to observations and simulations with the ADCIRCmodel (Dietrich et al, 2012). This is done in terms of peak surges, timing and the qualitative evolution. Next, a sensitivity analysis is carried out in which the storm parameters are systematically varied around the values that represent Hurricane Katrina.

\subsubsection{Outline of the thesis}

This thesis is organised as follows (also see Figure 1.9). After this introductory chapter, Chapter 2 deals with the resonance properties of a closed rotating basin subject to periodic wind forcing with different spatial patterns, thus addressing 

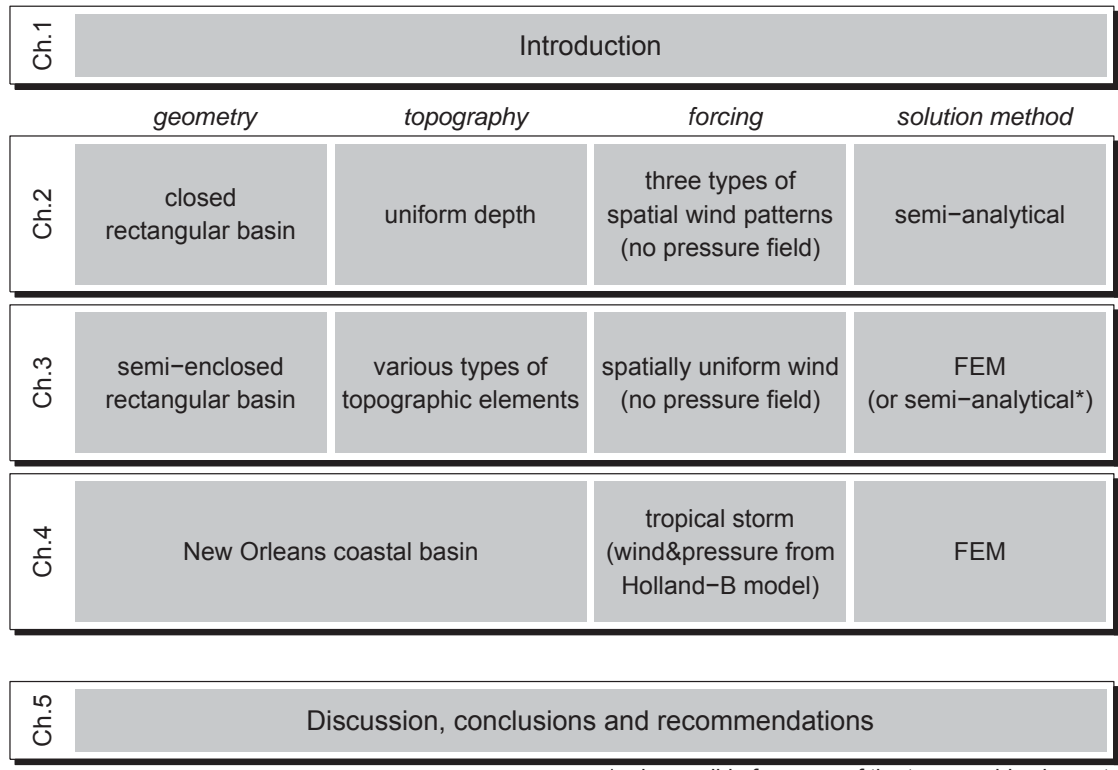

*only possible for some of the topographic elements

Figure 1.9: Outline of the thesis, characterising Chapters 2, 3 and 4 regarding geometry, topography, forcing and solution method. Here, FEM stands for Finite Element method.

research question Q1. Next, in Chapter 3, the influence of topographic elements on the resonance properties of a semi-enclosed coastal basin is considered (Q2). Subsequently, in Chapter 4, the influence of storm characteristics on surge level in the New Orleans coastal basin is studied (Q3). Finally, Chapter 5 contains the discussion, conclusions and recommendations. 


\section{Appendix}

\section{A Details of the simple wind-driven flow model}

This appendix contains the derivations of the equilibrium response to timeperiodic wind as well as the transient solution in the case of pure resonance, as discussed in $\S 1.3 .1$.

\section{A.1 Dynamic equilibrium solution}

First, the model equations in Eq.(1.2) and initial/boundary conditions can be transformed to the following problem for $\eta(x, t)$ only:

$$
\frac{\partial^{2} \eta}{\partial t^{2}}+\frac{r}{h} \frac{\partial \eta}{\partial t}-g h \frac{\partial^{2} \eta}{\partial x^{2}}=0, \quad \frac{\partial \eta}{\partial x}(0, t)=\frac{\partial \eta}{\partial x}(L, t)=\frac{\tau_{\mathrm{w}}^{(x)}}{\rho g h},
$$

with initial conditions $\eta(x, 0)=0$ and $\frac{\partial \eta}{\partial t}(x, 0)=0$.

A dynamic equilibrium solution follows from assuming

$$
\eta(x, t)=\Re\{N(x) \exp (-\mathrm{i} \omega t)\},
$$

with $\Re$ denoting the real part, $N(x)$ a complex surface elevation amplitude and $\mathrm{i}^{2}=-1$. In the absence of bottom friction $(r=0)$, solving for $N(x)$ gives the result presented in Eq.(1.4).

\section{A.2 Transient solution for pure resonance}

Now consider to the case of pure resonance $(r=0, k L=\tilde{n} \pi$ with $k=\omega / \sqrt{g h}$ for some odd $\tilde{n}$ ), for which the above equilibrium assumption fails. In this case, the focus is on the transient solution, which can be written as

$$
\begin{aligned}
& \text { (i) (ii) } \\
& \begin{aligned}
\eta(x, t)= & \overbrace{A_{\tilde{n}} \omega t \cos \left(\frac{\tilde{n} \pi x}{L}\right) \cos \omega t}^{(\mathrm{i})}+\overbrace{\sum_{\substack{n \text { odd } \\
n \neq \tilde{n}}}^{\infty} A_{n} \cos \left(\frac{n \pi x}{L}\right) \sin \omega t}^{(\mathrm{ii})} \\
& +\underbrace{\frac{\hat{T} L}{g h}\left[\frac{x}{L}-\frac{1}{2}\right] \sin \omega t}_{(\mathrm{iii})}+\underbrace{\sum_{\sum_{n \text { odd }}^{\infty} B_{n} \cos \left(\frac{n \pi x}{L}\right) \sin \omega_{n} t}^{\infty}}_{(\mathrm{iv})} .
\end{aligned}
\end{aligned}
$$

This expression consists of four terms: (i) an oscillatory harmonic profile with an amplitude that increases linearly with time (i.e., the term highlighted in the main text), (ii) a series of spatially harmonic profiles oscillating at constant amplitude, (iii) an oscillatory spatially linear profile, and (iv) a superposition of eigenmodes, such that the initial conditions are satisfied (with $\omega_{n}=$ $\sqrt{g h}(n \pi / L))$. The coefficients $A_{n}$ and $B_{n}$, defined for odd $n$, read

$$
A_{n}=\left\{\begin{array}{ll}
-\frac{1}{2} d_{\tilde{n}} & \text { if } n=\tilde{n}, \\
\frac{\tilde{n}^{2}}{n^{2}-\tilde{n}^{2}} d_{n} & \text { if } n \neq \tilde{n},
\end{array} \quad B_{n}= \begin{cases}\frac{1}{2} d_{\tilde{n}} & \text { if } n=\tilde{n} \\
-\frac{\tilde{n}^{2}}{n^{2}} d_{n} & \text { if } n \neq \tilde{n}\end{cases}\right.
$$


in which $d_{n}$ is a Fourier coefficient given by

$$
d_{n}=\frac{2}{L} \int_{0}^{L} \frac{\hat{T} L}{g h}\left[\frac{x}{L}-\frac{1}{2}\right] \cos \left(\frac{n \pi x}{L}\right) \mathrm{d} x=\frac{-4 \hat{T} L}{g h(n \pi)^{2}} .
$$

\section{A.3 Energy argument}

The total energy $E$ of our (linearised) system is the sum of the kinetic and potential energy, integrated over the basin:

$$
E=B \int_{0}^{L}\left[\frac{1}{2} \rho h \bar{u}^{2}+\frac{1}{2} \rho g \eta^{2}\right] \mathrm{d} x,
$$

where $B$ is basin width. From the model equations and boundary conditions it can be shown that the change of energy over time is given by

$$
\frac{\mathrm{d} E}{\mathrm{~d} t}=\underbrace{B \int_{0}^{L} \tau_{\mathrm{w}}^{(x)} \bar{u} \mathrm{~d} x}_{\text {(i) wind power }}-\underbrace{B \int_{0}^{L} \rho r \bar{u}^{2} \mathrm{~d} x}_{\text {(ii) dissipation }} .
$$

It consists of two parts: (i) wind power which is positive (negative) when the wind stress and flow velocity point in the same (opposite) direction, and (ii) dissipation due to bottom friction, which is zero when $r=0$.

Equation (1.13) refers to the instantaneous change of energy. To analyse resonance properties, we must consider the net change of energy over a wind cycle, defined as $\left\langle\frac{\mathrm{d} E}{\mathrm{~d} t}\right\rangle=\frac{1}{T} \int_{0}^{T} \frac{\mathrm{d} E}{\mathrm{~d} t} \mathrm{~d} t$ with $T=2 \pi / \omega$.

In pure resonance $(r=0, k L=\tilde{n} \pi)$, there is a net transfer of wind energy to the system, i.e. $\left\langle\frac{\mathrm{d} E}{\mathrm{~d} t}\right\rangle>0$. The energy is stored in the $\tilde{n}$-th mode, which has a linearly increasing amplitude.

In the presence of friction such an ever increasing amplitude is not possible. Instead, the system tends to a dynamic equilibrium in which dissipation balances the wind power input, i.e. $\left\langle\frac{\mathrm{d} E}{\mathrm{~d} t}\right\rangle=0$. This balance may produce high amplitudes at frequencies that slightly shifted with respect to the frequencies in pure resonance. This is termed practical resonance. 


\title{
Chapter 2
}

\section{Resonance properties of a closed rotating rectangular basin subject to space- and time-dependent wind forcing ${ }^{\star}$}

\begin{abstract}
We present an idealised process-based model to study the possibly resonant response of closed basins subject to periodic wind forcing. Two solution methods are adopted: a collocation technique (valid for arbitrary rotation) and an analytical expansion (assuming weak rotation). The spectral response, as obtained from our model, displays resonance peaks, which we explain by linking them to the spatial pattern of the wind forcing, the along-wind and cross-wind basin dimensions as well as the influence of rotation. Increasing bottom friction lowers the peaks. Finally, we illustrate how the spectral response is reflected in the time-dependent set-up due to a single wind event.
\end{abstract}

\subsection{Introduction}

Wind blowing over coastal basins often induces high water levels that may threaten coastal safety (Pugh, 1987). An overall practical goal is to be able to predict water levels for any type of wind event at any location. These water levels are generally sensitive to basin geometry and the type of wind forcing (Pugh, 1987). In extreme cases, a phenomenon known as resonance may occur. Examples of unusual flooding events that have been linked to such resonant conditions are typhoon Winnie at the Korean coast of the Yellow Sea (Moon et al, 2003) and storm Xynthia in the Bay of Biscay (Bertin et al, 2012). However, it is difficult to identify the physics from these complex site-specific events. Achieving the practical goal mentioned above requires a more generic insight in the physical processes underlying this wind-driven resonance phenomenon.

For the equilibrium response to steady wind, it is the relative importance of rotation and friction that determines the way in which the wind stress is communicated through the water column (e.g., Ekman, 1905; Csanady, 1982). This balance is expressed in the Ekman number $\delta_{E}=h^{-1} \sqrt{2 K / f}$ with water depth $h$, vertical eddy viscosity $K$ and Coriolis parameter $f$. Focusing on the

*This chapter has been published as Chen, W.L., Roos, P.C., Schuttelaars, H.M., and Hulscher, S.J.M.H. (2015). Resonance properties of a closed rotating rectangular basin subject to space- and time-dependent wind forcing., Ocean Dynamics, 165(3), doi:10.1007/s10236015-0813-2. 
circulation in closed basins, in shallow/highly turbulent basins (Mathieu et al, 2002; Winant, 2004) (small $\delta_{E}$ ) cross-wind flows are weak, whereas they are strong in deep/weakly turbulent basins (large $\delta_{E}$ ). The general case requires a three dimensional flow model.

Other studies focused on the time-dependency of the dynamics. Two approaches exist. The first is to study the transient evolution to equilibrium of a quiescent basin to a suddenly imposed spatially uniform wind (Csanady, 1968a; Birchfield, 1969; Mohammed-Zaki, 1980). The second is to study the response to a single wind event, characterised by not only a spin-up but also a spin-down stage. Such an event can be seen as the superposition of periodic wind forcings at various frequencies $\omega$ (Craig, 1989). Assuming linear dynamics, also the response will be the superposition of the responses at these individual frequencies. Hence, the basin's response to a single wind event lies in its spectral response. For example, from his idealised model for elongated basins $(B \ll L)$ subject to periodic and spatially uniform wind, Ponte (2010) identified resonance peaks associated with along-basin standing waves. The oscillations associated with these peaks (eigenmodes) were investigated more generally by Rao (1966). His numerical study particularly demonstrated that the resonant frequencies strongly depend on $B$ and $L$.

Other studies account for spatial variations in the wind field, which have been observed e.g. in the Gulf of California (Ponte et al, 2012) and are known to affect the response (Pugh, 1987). This was also found in theoretical studies, e.g. regarding the equilibrium response to steady wind in a shallow circular basin (Birchfield, 1967) and the transient response to a suddenly imposed wind stress in deep circular basins (Mohammed-Zaki, 1980).

From the above, we identify the following knowledge gap. There is no study systematically investigating the resonance properties of basins of arbitrary geometry, subject to arbitrary wind fields. The goal of the present study is to systematically investigate the resonance properties of wind-driven flow in closed rotating basins. Specifically, our research questions are as follows. How do the resonance properties depend on the following aspects: (1) basin dimensions, (2) the spatial structure of the wind forcing, and (3) bottom friction?

As a first step to answering these question, we present a three-dimensional idealised process-based model of wind-driven flow in closed rectangular rotating basins of uniform depth. The vertical profile of the flow field is resolved fully analytically, and expressed in the free surface elevation. In turn, the free surface elevation pattern follows from solving an elliptic problem. To solve it, two methods are used: $(i)$ a so-called collocation method, valid for arbitrary values of the dimensionless Coriolis parameter $f / \omega,(i i)$ an analytical approximation valid for small values of $f / \omega$ to obtain physical insight in the influence of rotation. Spatial variations in the wind are accounted for in a schematised way, i.e. by allowing linear variation of wind stress amplitude and phase in the along-wind (nonzero divergence) and cross-wind direction (nonzero curl).

This paper is organised as follows. In section 2.2, we present the model. Next, section 2.3 contains the solution method, and in section 2.4 we present the model results. Finally, sections 2.5 and 2.6 present the discussion and con- 
(a) top view

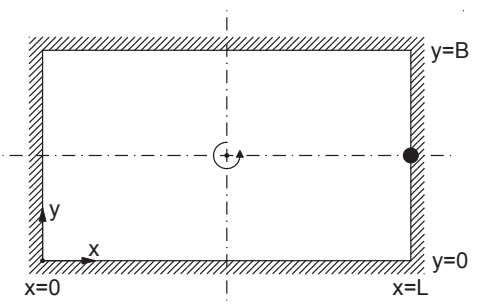

(b) side view (along-basin)

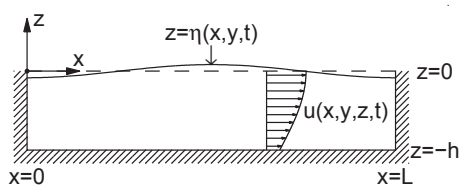

Figure 2.1: Definition sketch of the model geometry, showing a rectangular basin of uniform depth: $(a)$ top view, $(b)$ side view in along-basin direction displaying the vertical profile of one component of the three-dimensional flow field. The black dot in the left-hand image indicates the location used to evaluate the solution in $\$ 2.4$. The dash-dotted lines denote the along-basin and cross-basin centerlines, used in the symmetry arguments in $\S 2.5$.

clusions, respectively.

\subsection{Model formulation}

Consider a rectangular basin of length $L$, width $B$ and uniform depth $h$ on the $f$ plane (see Figure 2.1). Let $x$ and $y$ be the along-basin and cross-basin coordinates, such that the basin boundaries are located at $x=0, L$ and $y=0, B$. The vertical coordinate $z$ points upward, with $z=\eta$ denoting the free surface elevation with respect to the undisturbed water level $z=0$ and the bed level at $z=-h$. Let $\boldsymbol{u}=(u, v, w)$ represent the flow velocity vector, with components $u, v$ and $w$ in the $x, y$ and $z$-direction, respectively. Assuming that the vertical displacement of the free surface is small compared to the water depth, conservation of momentum and mass is expressed by the three-dimensional linearised shallow water equations according to

$$
\begin{aligned}
\frac{\partial u}{\partial t}-f v & =-g \frac{\partial \eta}{\partial x}+\frac{\partial}{\partial z}\left[K \frac{\partial u}{\partial z}\right], \\
\frac{\partial v}{\partial t}+f u & =-g \frac{\partial \eta}{\partial y}+\frac{\partial}{\partial z}\left[K \frac{\partial v}{\partial z}\right], \\
\frac{\partial u}{\partial x}+\frac{\partial v}{\partial y}+\frac{\partial w}{\partial z} & =0
\end{aligned}
$$

Here, $f=2 \Omega \sin \vartheta$ is the Coriolis parameter (with $\Omega=7.292 \times 10^{-5} \mathrm{rad} \mathrm{s}^{-1}$ the angular frequency of the Earth's rotation and $\vartheta$ the latitude), $g=9.81 \mathrm{~m} \mathrm{~s}^{-2}$ the gravitational acceleration and $K$ the vertical eddy viscosity, assumed constant. Horizontal mixing of momentum is neglected.

Regarding boundary conditions, we impose a wind stress at the free surface and a partial-slip condition at the bottom. Along with the kinematic boundary 
(a) spatially uniform part

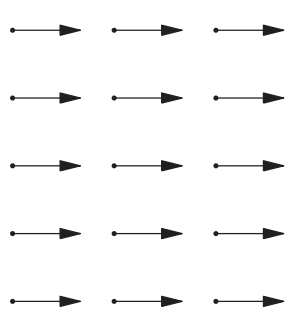

(b) divergent part

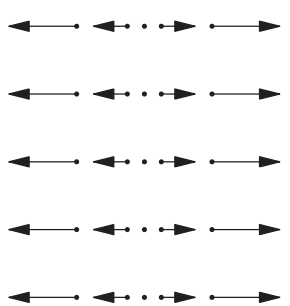

(c) curl part

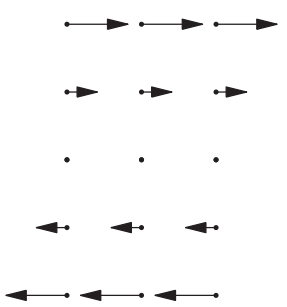

Figure 2.2: Top view of the three contributions to the spatial wind pattern in Eq.(2.7): (a) spatially uniform part, $(b)$ divergent part showing linear variation in the along-wind direction, $(c)$ curl part showing linear variation in the cross-wind direction. These images are snapshots showing wind directions at a certain time: half a period later these directions are reversed.

conditions, this reads in linearised form:

$$
\begin{array}{lll}
w=\frac{\partial \eta}{\partial t}, & K\left(\frac{\partial u}{\partial z}, \frac{\partial v}{\partial z}\right)=\frac{\left(\tau_{\mathrm{w}}^{(x)}, \tau_{\mathrm{w}}^{(y)}\right)}{\rho} \quad \text { at } z=0, \\
w=0, & K\left(\frac{\partial u}{\partial z}, \frac{\partial v}{\partial z}\right)=s(u, v) & \text { at } z=-h .
\end{array}
$$

The linearisation procedure causes the free surface condition to be imposed at $z=0$ instead of at $z=\eta$. In Eq.(2.5), we have introduced the resistance parameter $s$, its value usually obtained from the analysis of field data. Two limiting cases are of interest. For large $s$, the bottom boundary condition effectively means no-slip, as used by e.g. Ponte (2012). On the other hand, $s=0$ corresponds to free-slip for which the flow becomes $z$-independent.

Furthermore, $\left(\tau_{\mathrm{w}}^{(x)}, \tau_{\mathrm{w}}^{(y)}\right)$ is the wind stress vector. The wind is assumed timeperiodic with angular frequency $\omega$, aligned with the $x$-direction. In addition to a spatially uniform contribution (Figure $2.2 a$ ), we allow the wind to vary linearly in both the along-wind and the cross-wind direction. Along-wind variations lead to a nonzero divergence of the wind field, cross-wind variations to a nonzero curl (see Figs.2.2b,c). This means

$$
\frac{\left(\tau_{\mathrm{w}}^{(x)}, \tau_{\mathrm{w}}^{(y)}\right)}{\rho}=\Re\left\{\left(T^{(x)}, T^{(y)}\right) \exp (-i \omega t)\right\},
$$

with

$$
T^{(x)}=\hat{T}\left(1+a\left[\frac{2 x}{L}-1\right]+b\left[\frac{2 y}{B}-1\right]\right), \quad T^{(y)}=0 .
$$

The parameter $\hat{T}$ denotes the magnitude of the forcing (wind stress divided by density) at the basin's centre. The complex coefficients $a$ and $b$ quantify 
the along-wind and cross-wind variation from this basin-averaged value. Importantly, the assumption of wind in $x$-direction only is not restrictive. The solution to a periodic wind in an arbitrary direction is the superposition of the separate solutions for wind in $x$ - and $y$-direction only. The latter solution can be obtained by rotating the entire system 90 degrees in the clockwise direction (effectively interchanging $L$ and $B$, wind now parallel to $x$-axis) and finally rotating the solution 90 degrees in the counterclockwise direction.

Finally, at the horizontal boundaries of the rectangular basin we require the normal transports to vanish, i.e.

$$
\langle u\rangle=0 \quad \text { at } x=0, L \quad \text { and } \quad\langle v\rangle=0 \quad \text { at } y=0, B .
$$

where angle brackets denote vertical integration from bottom to surface, i.e. $\langle\cdot\rangle=\int_{-h}^{0} \cdot \mathrm{d} z$ (with the upper boundary $z=0$ arising from the linearisation).

\subsection{Solution method}

\subsubsection{Differential problem for surface elevation amplitude}

First we write the solution in a time-periodic fashion according to

$$
\begin{aligned}
& \eta=\Re\{N(x, y) \exp (-i \omega t)\}, \\
& u=\Re\{U(x, y, z) \exp (-i \omega t)\},
\end{aligned}
$$

with complex amplitudes $N$ and $U$. Similar expression hold for $v$ and $w$, with complex amplitudes $V$ and $W$.

Next, we express the horizontal flow solution $\tilde{u}$ and $\tilde{v}$ in terms of surface slopes $\nabla \eta$ and wind stress. This is done using so-called rotating flow components, for which we derive expressions; see Appendix 2.A.1. Substituting these expressions into the continuity equation and integrating from bottom to surface gives the following elliptic equation for $N$ (see Appendix 2.A.2):

$$
\frac{\partial^{2} N}{\partial x^{2}}+\frac{\partial^{2} N}{\partial y^{2}}+k^{2} N=-\left[\frac{\partial\left\langle R_{1}\right\rangle}{\partial x}+\frac{\partial\left\langle R_{2}\right\rangle}{\partial y}\right],
$$

in which $k$ is a wave number satisfying

$$
k^{2}=\frac{-i \omega}{\left\langle C_{1}\right\rangle}
$$

with the coefficient $\left\langle C_{1}\right\rangle$ as specified in Appendix 2.A.2. The forcing term on the right-hand side of Eq.(2.11) includes contributions arising from the divergence and curl of the wind stress field (see Appendix 2.A.1); it is zero for spatially uniform wind. Moreover, $k$ is a wave number, and the coefficient $\left\langle C_{1}\right\rangle$ in Eq.(2.12) is as specified in Appendix 2.A.2.

The boundary conditions in Eq.(2.8) imply

$$
\begin{gathered}
\frac{\partial N}{\partial x}+\gamma \frac{\partial N}{\partial y}=-\left\langle R_{1}\right\rangle \quad \text { at } x=0, L, \\
-\gamma \frac{\partial N}{\partial x}+\frac{\partial N}{\partial y}=-\left\langle R_{2}\right\rangle \quad \text { at } y=0, B,
\end{gathered}
$$


with coefficient $\gamma=\left\langle C_{2}\right\rangle /\left\langle C_{1}\right\rangle$ as well as forcing terms $\left\langle R_{1}\right\rangle$ and $\left\langle R_{2}\right\rangle$ associated with the wind stress at the cross-basin and along-basin boundaries, respectively; see Appendix 2.A.2. Finally, the vertical flow amplitude $W$ at any depth $z$ can be expressed in terms of the free surface elevation $N$ and the wind forcing. This follows from integration of the continuity equation; see Appendix 2.A.3.

Finally, for $\omega=0$, the wave number in Eq.(2.11) reduces to $k^{2}=0$, and as an additional condition the total water volume in the basin must be prescribed.

\subsubsection{Collocation method}

The solution, for arbitary values of the dimensionless rotation parameter $f / \omega$, will be written as

$$
N=N^{\text {unif }}+a N^{\text {div }}+b N^{\text {curl }}
$$

with three contributions associated with the spatially uniform part of the wind, the divergent part of the wind $(a \neq 0)$ and the curl part of the wind $(b \neq 0)$.

The first contribution $N^{\text {unif }}$ takes advantage of the fact that a function $\phi^{\text {unif }}(y)$ exists satisfying both the differential equation (2.11) and the alongbasin boundary conditions in Eq.(2.14), only regarding the forcing terms arising from the spatially uniform wind. See Appendix 2.B.1 for an expression for $\phi_{\text {unif }}$. We thus write

$$
N^{\text {unif }}=\phi^{\text {unif }}(y)+\sum_{m=0}^{M} c_{m}^{\oplus} N_{m}^{\oplus}(x, y)+\sum_{m=0}^{M} c_{m}^{\ominus} N_{m}^{\ominus}(x, y),
$$

with $N_{m}^{\oplus}(x, y)$ and $N_{m}^{\ominus}(x, y)$ representing two families of so-called along-basin eigenmodes, consisting of Kelvin and Poincaré modes propagating or exponentially decaying in the positive or negative $x$-direction, respectively (see Appendix 2.B.3). In Eq.(2.16), $M$ is the truncation number. The coefficients $c_{m}^{\oplus}$ and $c_{m}^{\ominus}$ follow from applying a collocation technique. Herein, we require the boundary condition (2.13) to be satisfied at two sets of $M+1$ collocation points, one at $x=0$ and the other at $x=L$ (see Figure 2.3$)^{\star}$. Note that this boundary condition includes a contribution due to $\phi^{\text {unif }}$.

The second contribution $N^{\text {div }}$ is found analogously, but now using a solution $\phi^{\text {div }}(x)$ satisfying both the differential equation and the cross-basin boundary conditions, regarding the forcing terms proportional to a (see Appendix 2.B.1). We thus write

$$
N^{\operatorname{div}}=\phi^{\operatorname{div}}(x)+\sum_{m=0}^{\tilde{M}} a_{m}^{\oplus} \tilde{N}_{m}^{\oplus}(x, y)+\sum_{m=0}^{\tilde{M}} a_{m}^{\ominus} \tilde{N}_{m}^{\ominus}(x, y),
$$

Also this solution involves two families of cross-basin eigenmodes, but now propagating or exponentially decaying in the positive or negative $y$-direction, respectively. The $2(\tilde{M}+1)$ collocation points are now located at $y=0$ and $y=B$. Please note that the value of $\tilde{M}$ may differ from the value of $M$ used above.

\footnotetext{
*This approach of finding solutions involving multiple sets of collocation points, has been adopted earlier in a tidal flow context (Roos and Schuttelaars, 2011; Roos et al, 2011).
} 
(a) $\mathrm{N}^{\text {unif }}$ and $\mathrm{N}^{\text {curl }}$ using along-basin modes

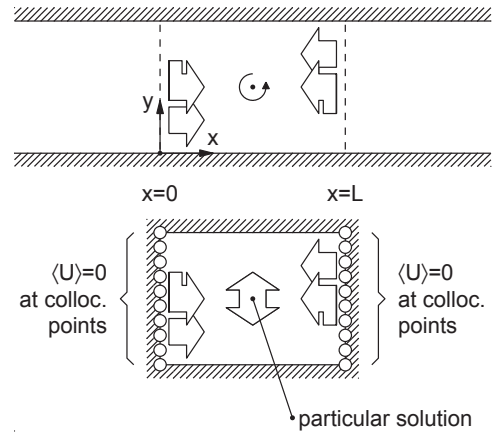

(b) $\mathrm{N}^{\text {div }}$ using cross-basin modes*

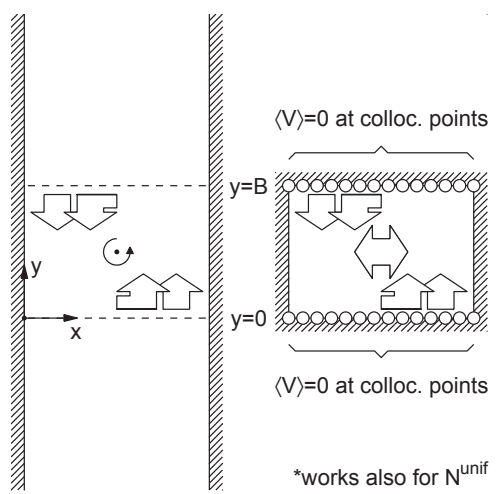

Figure 2.3: Sketch of the solution method outlined in $\S 2.3 .2$. (a) To calculate $N^{\text {unif }}$ and $N^{\text {curl }}$, a superposition of along-basin channel modes (white arrows) and a suitable particular solution (double arrow) is forced to satisfy $\langle U\rangle=0$ at a set of collocation points at $x=0, L$ (white circles). (b) Alternatively, to obtain $N^{\text {div }}$, a superposition of cross-basin channel modes and a particular solution is forced to satisfy $\langle V\rangle=0$ at a set of collocation points at $y=0, B$. By symmetry, the latter orientation also works for $N^{\text {unif }}$, which is done in the convergence test (Appendix 2.B.2).

Finally, the third contribution reads

$$
N^{\text {curl }}=\phi^{\text {curl }}(y)+\sum_{m=0}^{M} b_{m}^{\oplus} N_{m}^{\oplus}(x, y)+\sum_{m=0}^{M} b_{m}^{\ominus} N_{m}^{\ominus}(x, y),
$$

again involving along-basin eigenmodes and collocation points identical to those used in obtaining $N^{\text {unif }}$ (and involving $\phi^{\text {curl }}$, as given in Appendix 2.B.1).

By symmetry, the contribution $N^{\text {unif }}$ due to the spatially uniform wind can just as well be obtained with an alternative form of Eq.(2.16), involving crossbasin eigenmodes and collocation points at $y=0$ and $y=B$. This requires an alternative particular solution $\tilde{\phi}^{\text {unif }}(x)$ instead of $\phi^{\text {unif }}(y)$. This symmetry property provides us with an opportunity to perform a convergence test; see Appendix 2.B.2. This possibility does not exist for the other two contributions $N^{\text {div }}$ and $N^{\text {curl }}$.

\subsubsection{Analytical solution for small $f / \omega$ and free slip}

In the case of weak Coriolis effects, we may obtain additional insight from expanding the solution in powers of $\epsilon=f / \omega$. For convenience we will do this for the case of free $\operatorname{slip}(s=0)$, which makes the flow pattern $z$-independent. Let us write

$$
\begin{aligned}
& N=N_{0}+\epsilon N_{1}+\epsilon^{2} N_{2}+\ldots, \\
& U=U_{0}+\epsilon U_{1}+\epsilon^{2} U_{2}+\ldots
\end{aligned}
$$


and the same for $V$, where $U$ and $V$ are the flow velocities.

At lowest order, i.e. at $\mathcal{O}\left(\epsilon^{0}\right)$, Coriolis effects is absent. For the uniform and divergent wind field, we then obtain a purely along-basin oscillation. Alternatively, due to its cross-basin dependency, the solution due to the curl-part of the wind also displays cross-basin oscillations. Adopting a notation that is convenient to present also the higher order solutions, the lowest order solution reads

$$
\begin{aligned}
& \frac{N_{0}}{\hat{N}}=F_{k_{0}}^{-}(x)+a\left[\frac{2}{k_{0} L}-F_{k_{0}}^{+}(x)\right]+b \sum_{m \text { odd }} c_{m} F_{\tilde{\alpha}_{m}}^{-}(x) \cos \beta_{m} y \\
& \frac{U_{0}}{\hat{U}}=i G_{k_{0}}^{-}(x)+i a\left[\frac{2 x}{L}-G_{k_{0}}^{+}(x)\right] \\
& \quad+i b \sum_{m \text { odd }} c_{m} \frac{\tilde{\alpha}_{m}}{k_{0}} G_{\tilde{\alpha}_{m}}^{-}(x) \cos \beta_{m} y \\
& \frac{V_{0}}{\hat{U}}=i b \sum_{m \text { odd }} c_{m} \frac{\beta_{m}}{k_{0}} F_{\tilde{\alpha}_{m}}^{-}(x) \sin \beta_{m} y .
\end{aligned}
$$

Here we have introduced reference values of the elevation and velocity amplitudes given by $\hat{N}=\hat{T} /\left(g h k_{0}\right)$ (with shallow water wave number $k_{0}=\omega / \sqrt{g h}$ ) and $\hat{U}=\hat{T} /(\omega h)$, respectively. Next, the curl-part has a cross-basin structure containing a cross-basin wave number $\beta_{m}=m \pi / B$ and coefficients $c_{m}=$ $-8 k_{0} /\left[\tilde{\alpha}_{m}(m \pi)^{2}\right]$, only required for odd values of $m$. Moreover, we used the dimensionless functions $F_{k_{0}}^{ \pm}(x)=\sin k_{0} x \pm \xi_{k_{0} L}^{ \pm} \cos k_{0} x$ and $G_{k_{0}}^{ \pm}(x)=1-\cos k_{0} x \pm$ $\xi_{k_{0} L}^{ \pm} \sin k_{0} x$ involving the coefficient $\xi_{k_{0} L}^{ \pm}=\left(1 \pm \cos k_{0} L\right) / \sin k_{0} L$. Similarly, we introduce along-basin wave numbers $\tilde{\alpha}_{m}$ satisfying $\tilde{\alpha}_{m}^{2}+\beta_{m}^{2}=k_{0}^{2}$ as well as functions $F_{\tilde{\alpha}_{m}}^{ \pm}(x)$ and $G_{\tilde{\alpha}_{m}}^{ \pm}(x)$ involving a similar coefficient $\xi_{\tilde{\alpha}_{m} L}$.

The solutions at first order, i.e. at $\mathcal{O}\left(\epsilon^{1}\right)$ and $\mathcal{O}\left(\epsilon^{2}\right)$ express how Coriolis effects modify the above oscillations. Mathematically, the Coriolis acceleration of the lowest order flow enters as the only forcing term at first order (the direct wind forcing being absent here). As a result, the solutions for a uniform and divergent wind field now experience a forcing in the cross-basin direction. Alternatively, the curl-part is further modified in along-basin and cross-basin directions. Expressions for the first order solutions are given in Appendix 2.C.

\subsection{Results}

\subsubsection{Collocation method}

To present the results, we consider a reference basin with characteristics as shown in Table 2.1. This corresponds to a basin with dimensions roughly representing those of the Southern Bight of the North Sea. To investigate the role of bottom friction, we consider a frictional case with the resistance parameter equal to $s=10^{-4} \mathrm{~m} \mathrm{~s}^{-1}$ as well as a frictionless case with $s=0$. 
Table 2.1: Overview of model parameters and their reference values.

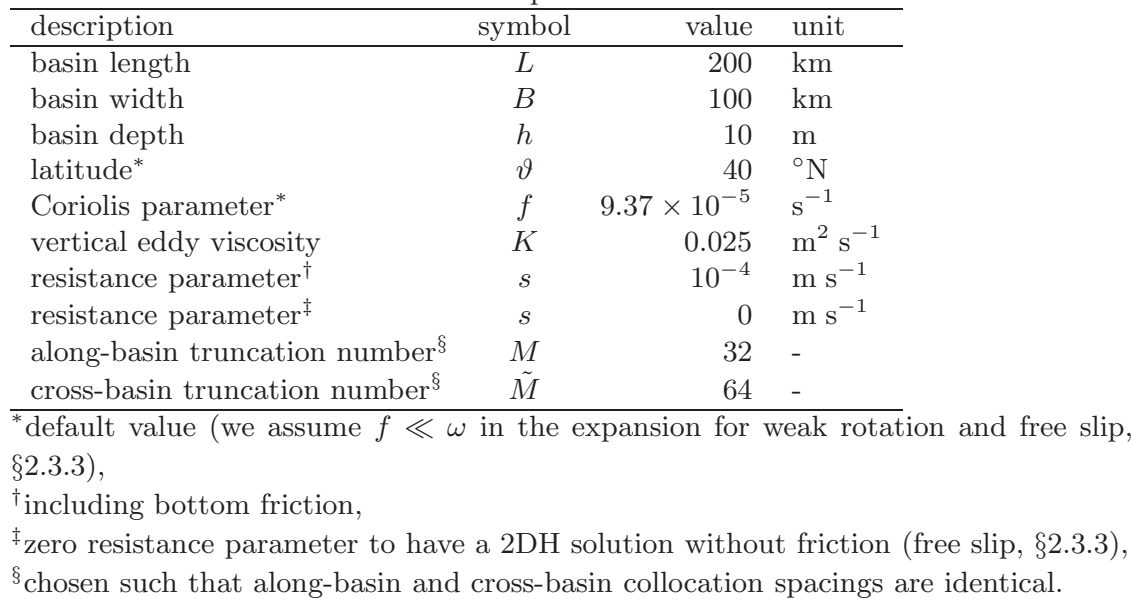

We will first present three examples (Fig.2.4). The examples all deal with the reference basin, but differ with respect to the applied forcing. We have intentionally chosen our forcing frequencies such that they coincide with peaks in the spectral response, to be shown in Fig.2.5. The first example is forced by a spatially uniform wind field with an angular frequency given by $\omega_{1}=$ $1.52 \times 10^{-4} \mathrm{rad} \mathrm{s}^{-1}=0.49 \omega_{\text {ref }}$ with reference frequency

$$
\omega_{\mathrm{ref}}=\sqrt{g h} \frac{2 \pi}{L}
$$

The reference frequency is the frequency for which the frictionless shallow water wavelength equals the basin length. The second example is forced by a spatially uniform wind field, as well, but now of angular frequency $\omega_{2}=$ $2.95 \times 10^{-4} \mathrm{rad} \mathrm{s}^{-1}=0.95 \omega_{\text {ref }}$. The same frequency is applied in the third example, but now using a divergent wind field. Since the solution is time-periodic, the elevation field can be visualised as an amphidromic system, displaying amplitudes and co-phase lines. As shown in Fig.2.4a, the first examples display a rotating Kelvin wave with one amphidromic point at the basin center, and relatively high amplitudes near the coast. The other examples, shown in Figs.2.4bc, display entirely different patterns and much lower amplitudes. These examples already illustrate that the response depends on both type of forcing and forcing frequency.

To further investigate the dependency of the solution on the forcing frequency for the various types of wind forcing, we will focus on the location midway one of the cross-basin boundaries; see the black dot in Fig.2.1 a. The elevation amplitude at this location, scaled against the reference elevation am- 
(a) uniform wind, $\omega=\omega_{1}$

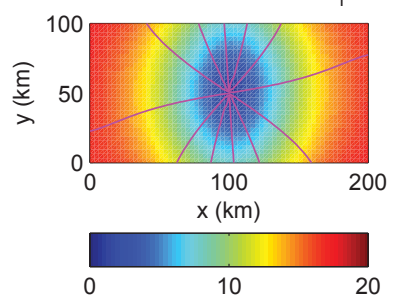

(b) uniform wind, $\omega=\omega_{2}$

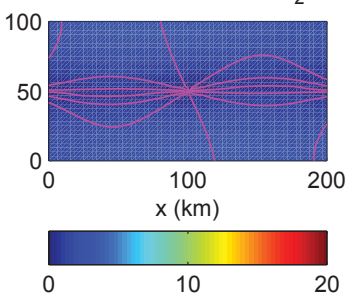

(c) divergent wind, $\omega=\omega_{2}$

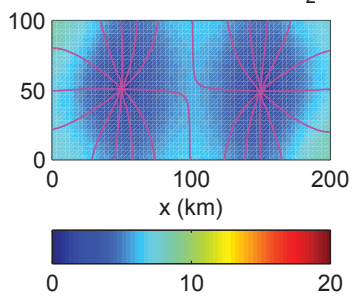

Figure 2.4: Amphidromic charts for three examples of basin responses: (a) uniform wind with $\omega=\omega_{1},(b)$ uniform wind with $\omega=\omega_{2},(c)$ divergent wind with $\omega=\omega_{2}$. Colors indicate elevation amplitude; pink lines are the co-phase lines. Parameter values as in Table 2.1 (including bottom friction).

plitude $\hat{N}$ according to

$$
A=\frac{|N|}{\hat{N}} \quad \text { at }(x, y)=\left(L, \frac{1}{2} B\right) \text { with } \hat{N}=\frac{\hat{T}}{g h k_{0}},
$$

will be used to evaluate the solution. Figure 2.5 shows the value of $A$ as a function of the dimensionless forcing frequency $\omega / \omega_{\text {ref }}$. This is done for each of the three types of wind forcing, in each case distinguishing a case without (black line) and with bottom friction (thick pink line). First of all, the three examples presented before appear here as part of the spectral response, the first one clearly having the highest value. More generally, the spectral response shows a complex pattern of peaks at certain frequencies and lower responses in between. Comparison between Figs.2.5abc shows that this pattern strongly depends on the type of wind forcing, e.g. at $\omega \approx \frac{1}{2} L / \lambda$ showing a peak for the spatially uniform wind, but not for the other types of forcing.

Increasing the resistance parameter (see Table 2.1) generally lowers the peaks, in certain cases causing it more or less to disappear. As a second order effect, actually not visible in Fig.2.5, the peaks shift to a slightly lower frequency. Further simulations, not shown here, suggest that the spectral response of the surface elevation hardly depends on the value of the vertical eddy viscosity $K$. However, the vertical structure of the flow velocity does depend on $K$.

The influence of basin width on the spectral response is shown in Fig.2.6, for each of the three types of wind forcing. We kept the basin length to its default value, and varied basin width from $B=25 \mathrm{~km}$ to $400 \mathrm{~km}$, thus covering a widthover-length range from 0.1 to 3.5. For the solutions with collocation points along the cross-basin boundaries, the number of collocation points was adjusted to keep a collocation spacing to a constant value of about $3 \mathrm{~km}$. The colour plots show that for elongated basins, i.e. for $B / L \ll 1$, the spectral response is nearly $B / L$-independent for the frequency range plotted. In Fig. $2.6 a$, we particularly reproduce the peaks mentioned by Ponte (2010); and we now show that similar behavior is found for the other spatial wind patterns. For non-elongated basins, 
(a) spatially uniform part

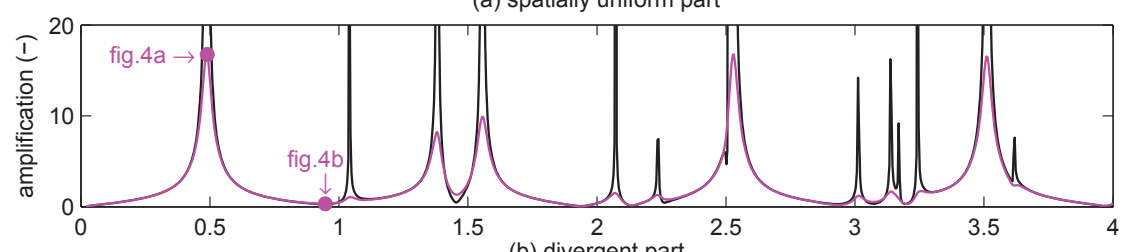

(b) divergent part
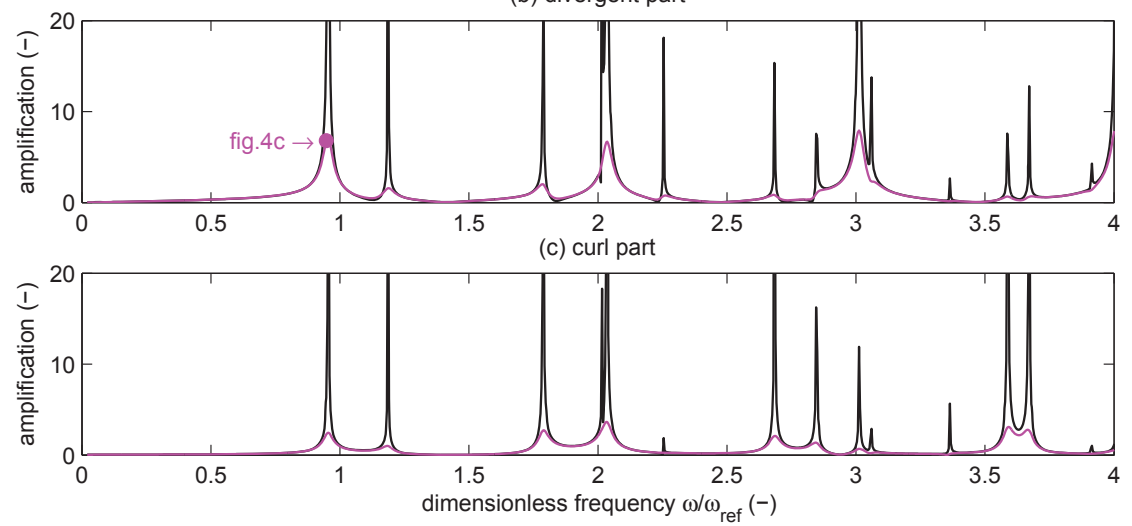

Figure 2.5: Scaled elevation amplitude $A$ at evaluation point (black dot in Fig.2.1a), as a function of the dimensionless forcing frequency $\omega / \omega_{\text {ref }}$ for three different types of wind forcing: (a) spatially uniform part, $(b)$ divergent part, $(c)$ curl part. Parameter values as in Table 2.1, with black and pink curves pertaining to the cases without and with bottom friction, respectively. As indicated, the three pink dots refer to the cases displayed in Fig.2.4. Please note that all peaks of the black curve should reach to infinity, but due to the plotting resolution for $\omega$ this is not visible for all peaks. 


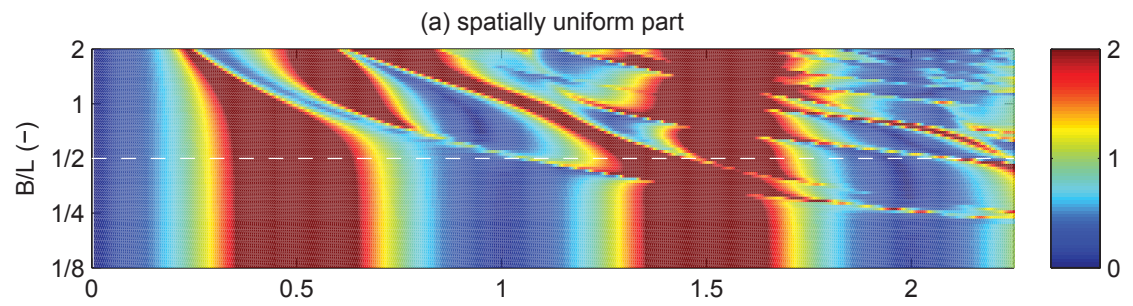

(b) divergent part

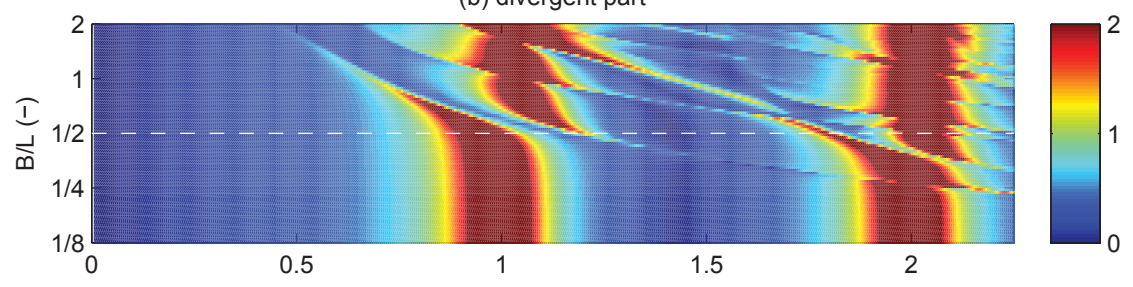

(c) curl part

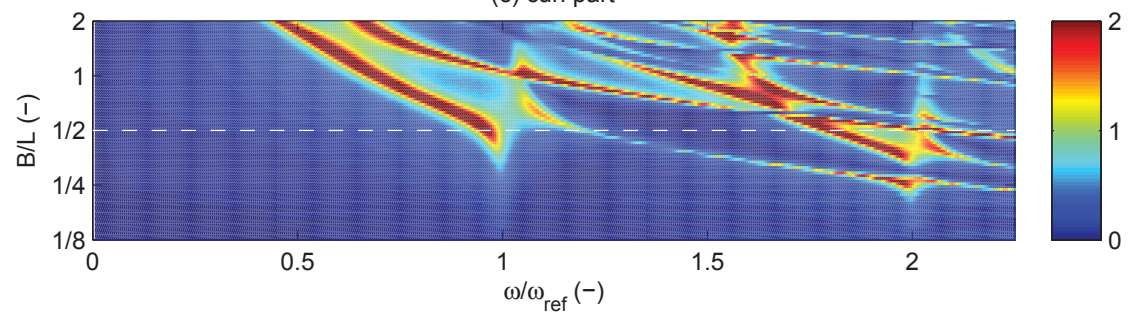

Figure 2.6: Scaled elevation amplitude $A$ at evaluation point (black dot in Fig.2.1a), as a function of dimensionless forcing frequency $\omega / \omega_{\text {ref }}$ and dimensionless basin width $B / L$, for three different types of wind forcing: $(a)$ spatially uniform part, $(b)$ divergent part, $(c)$ curl part. This figure has been obtained by varying $\omega$ and $B$, while fixing the other parameters to the values listed in Table 2.1 (including bottom friction).

i.e. for $B / L=\mathcal{O}(1)$ and larger, a more complicated pattern is obtained showing a strong width-dependence to be further interpreted in $\S 2.5 .2$. The reference case for which $B / L=\frac{1}{2}$ is denoted by a dashed white line.

\subsubsection{Analytical solution for small $f / \omega$ and free slip}

In addition to the results from the collocation method, we now turn to the analytical solution in powers of $f / \omega$. The spectral response at various orders of $\epsilon=f / \omega$ is shown in Fig.2.7 (for spatially uniform and divergent wind) and Fig.2.8 (for wind with nonzero curl), again as a function of the scaled frequency $L / \lambda$. Analogous to Rao (1966), the resonance peaks have been labeled by bracketed numbers $(m, n)$, with along-basin mode number $m$ and cross-basin mode number $n$. As such, they correspond to an eigenmode with a specific spatial structure as presented by Rao (1966). For example, the mode associated with 
(a) lowest order: $\mathrm{O}\left(\varepsilon^{0}\right)$
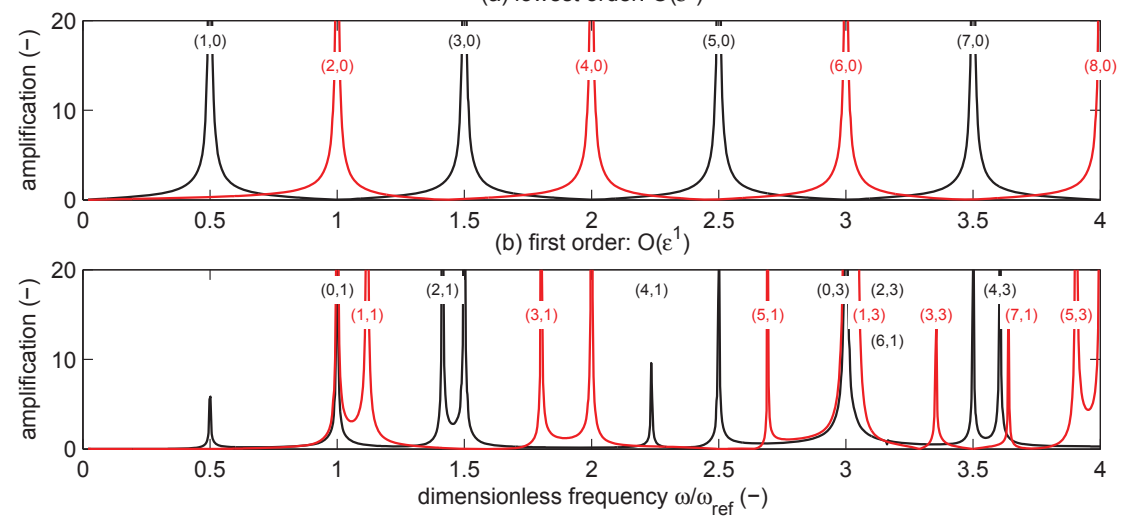

Figure 2.7: Scaled elevation amplitude $A$ at evaluation point (black dot in Fig.2.1a), as a function of the dimensionless forcing frequency $L / \lambda$ according to the expansion in powers of $\epsilon=f / \omega$ : (a) lowest order, (b) first order. The curves pertain to the solution due to the spatially uniform part (black) and the divergent part (red) of the wind field. Bracketed numbers $(m, n)$ indicate the eigenmode with along-basin mode number $m$ and cross-basin mode number $n$; see text. Parameter values as in Table 2.1 (free slip case and small $f$ ).

the $(1,0)$-peak at $L / \lambda=\frac{1}{2}$ in Fig. $2.7 a$ is actually the frictionless counterpart of the first example (Fig.2.4a), in the limit of no rotation. Likewise, the mode associated with the $(2,0)$-peak at $L / \lambda=1$ in Fig. $2.7 \mathrm{a}$ is the counterpart of the third example (Fig.2.4c). Peaks are labeled only if they are new at a certain order in $\epsilon$, not if they are already present at a lower order for the same type of forcing. As noted by Rao (1966), all eigenmodes are either symmetric or antisymmetric about the center point of the basin.

\subsection{Discussion}

\subsubsection{Interpretation of the resonances}

To interpret the complex patterns of peaks in the spectral response presented in $\S 2.4 .1$, we will examine the physics behind the peaks appearing in the analytical solution for small $f / \omega$ and free slip presented in $\S 2.4 .2$. To this end, we must turn to the concept of resonance. Resonance implies that, when starting from rest, the forcing continuously feeds energy into the system. This means that the net power supplied by the wind forcing, i.e. integrated over the basin and averaged over a forcing period, is positive. In our context with along-basin wind only and neglecting dissipation, this resonance condition can be expressed as

$$
\mathcal{P}_{\text {input }}=\frac{\omega}{2 \pi} \int_{0}^{2 \pi / \omega}\left[\rho \int_{0}^{B} \int_{0}^{L} u \tau_{\mathrm{w}} \mathrm{d} x \mathrm{~d} y\right] \mathrm{d} t>0 .
$$


(a) lowest order: $O\left(\varepsilon^{0}\right)$

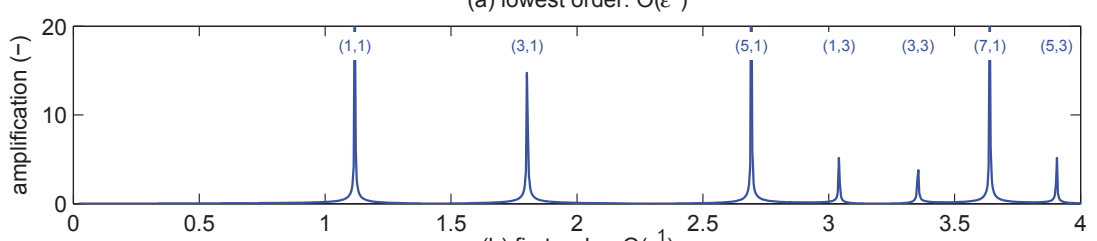

(b) first order: $\mathrm{O}\left(\varepsilon^{1}\right)$

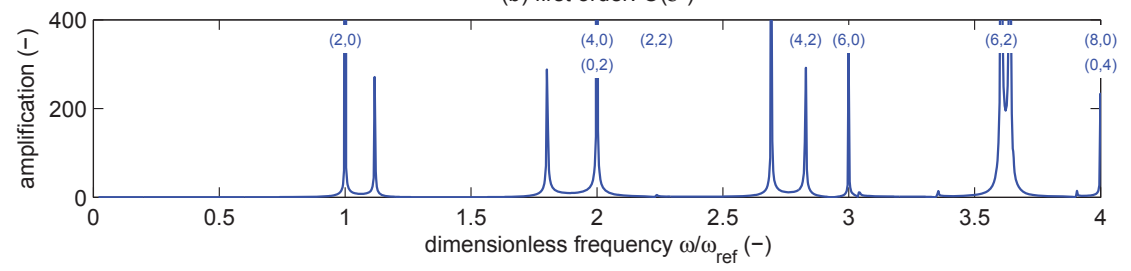

Figure 2.8: Same as Fig.2.7, but now for the solution due to the curl part of the wind field (blue), which is available at $(a)$ the lowest order and $(b)$ the first order.

Whether a certain mode can be excited depends on symmetry properties associated with the spatial structure of the wind field in relation to the spatial structure of the along-basin flow field.

Let us now focus on the lowest order solution for spatially uniform and divergent wind (Fig.2.7a), i.e. without rotation. The peaks occurring at $L / \lambda=$ $\frac{1}{2}+p$ for $p=0,1,2, \cdots$, associated with the odd modes $(2 p+1,0)$ are the odd seiches of a closed basin that can be excited by a spatially uniform wind (Csanady, 1982; Ponte, 2010). In addition to this well known result, the peaks at $L / \lambda=p$ for $p=1,2, \cdots$ demonstrate that a divergent wind field may excite the even modes $(2 p, 0)$. Symmetry arguments help to explain this from Eq.(2.26). Odd modes can be excited by a spatially uniform wind field, because both wind and along-basin flow are symmetric about the cross-basin centerline, thus giving a nonzero result in Eq.(2.26). Analogously, even modes can be excited by a divergent wind field, because both wind and along-basin flow are then antisymmetric about the cross-basin centerline. This is illustrated in Fig.2.9a,b.

Next, let us discuss the lowest order solution due to the curl part of the wind field, also without rotation. As shown by the peaks in Fig.2.8a, modes characterised by odd along-basin and odd cross-basin mode numbers can be resonant under this type of forcing. The following symmetry arguments show why this is the case. Modes with an even along-basin mode number cannot be resonant, because the along-basin flow on the left-hand side of the cross-basin centerline is then always opposite to that on the right-hand side. However, the wind forcing is symmetric about this cross-basin centerline. The power input by the wind in these two parts of the basin will thus always cancel, which causes the net power by the wind forcing to be zero. A similar argument holds for the modes with an even cross-basin mode number, but now with respect to the along-basin 

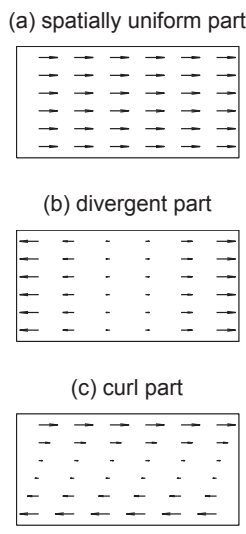

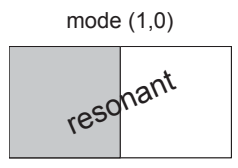

mode $(1,0)$
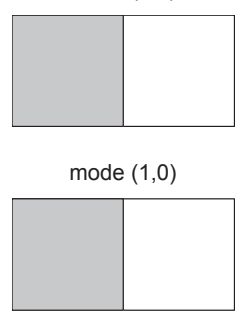

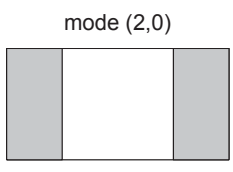

mode $(2,0)$

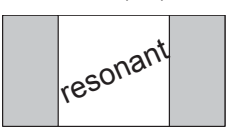

mode $(2,0)$

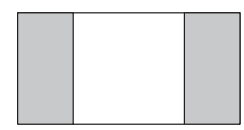

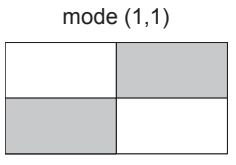

mode $(1,1)$
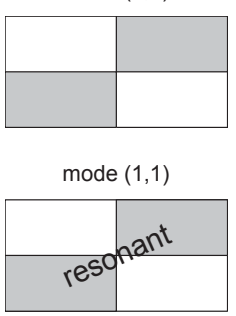

Figure 2.9: Sketch of the symmetry argument showing the spatial structure of the forcing (left-hand panel) and the spatial structure of the along-basin flow field of three modes: $(1,0),(2,0)$ and $(1,1)$. Grey shades indicate positive values, white means negative. We consider three types of wind forcing: $(a)$ spatially uniform part, $(b)$ divergent part and $(c)$ curl part. Whether a certain mode is resonant under these forcing conditions, is denoted in the figure.

centerline. These modes cannot be resonant, because the along-basin flow is then symmetric about this line and the wind forcing is antisymmetric about this line. Hence, the power input by the wind in these situations. Such symmetry arguments do not apply to the modes characterised by odd along-basin and odd cross-basin mode numbers. The above reasoning is illustrated in Fig.2.9c.

The first order solutions for the uniform and divergent wind case show new resonances; see the peaks in Fig.2.7b. To understand these peaks, it is crucial to realise that the first order problem is actually similar to the lowest order problem, but instead of by the wind it is forced by the Coriolis acceleration of the lowest-order along-basin flow. This forcing acts in the cross-basin direction, it is uniform in this cross-basin direction and it has an along-basin structure that depends on the type of wind forcing. For spatially uniform wind, the lowest order along-basin flow in Eq.(2.22) and hence the first order forcing is symmetric about the cross-basin centerline. Alternatively, for divergent wind, the first order forcing is antisymmetric about the cross-basin centerline. Symmetry arguments similar to those presented above for the lowest order solution due to the curlpart now explain why the uniform wind (divergent wind) gives rise to resonance at modes with an even (odd) along-basin mode number, and in each case an odd cross-basin mode number.

Similar symmetry arguments explain the new peaks arising in the first order response to the curl part of the wind (Fig.2.8b), but this is more complicated due to the spatial structure of the forcing. 


\subsubsection{Influence of basin dimensions}

The modes identified in the previous subsection allow us to interpret the widthdependence of the (peaks in the) spectral response presented in Fig.2.6. As noted earlier, for elongated basins, i.e. for $B<L \ll 1$, the response is independent of $B / L$ in the frequency range under consideration. This is because the cross-basin dynamics are weak. For non-elongated basins, i.e. for $B / L=\mathcal{O}(1)$ and larger, we see that these purely along-basin modes (e.g., $(1,0)$ for the spatially uniform wind) are modified by Coriolis effects into a more rotary wave propagating around the basin, which has a longer travel distance and thus a lower resonant frequency. In addition to this, resonant peaks appear pertaining to purely crossbasin modes (e.g., $(0,1))$ as well as mixed along-/cross-basin modes (e.g., $(2,1)$ ). As can be expected, the peak frequency modes decrease with increasing basin width. In conclusion, we can say that for non-elongated basins, cross-wind dynamics produces peaks at frequencies significantly lower than those obtained by Ponte (2010).

\subsubsection{Single wind event}

We will now illustrate how the spectral response translates into time-dependent elevation patterns for a single wind event (Fig. 2.10). To this end, it is important to realise that any wind event can be represented as the superposition of periodic signals. This means that Eq.(2.6) must be extended according to

$$
\frac{\left(\tau_{\mathrm{w}}^{(x)}, \tau_{\mathrm{w}}^{(y)}\right)}{\rho}=\Re\left\{\sum_{p=0}^{P}\left(T_{p}^{(x)}, T_{p}^{(y)}\right) \exp \left(-i \omega_{p} t\right)\right\},
$$

with frequencies $\omega_{p}=p \omega_{\min }$ and corresponding wind amplitudes $T_{p}^{(x)}$ and $T_{p}^{(y)}$. As we must discretise the wind spectrum, there is a minimum frequency $\omega_{\min }$ and hence a recurrence period $T_{\text {recur }}=2 \pi / \omega_{\text {min }}$ over which the event repeats itself. To realistically describe a 'single' wind event, this recurrence period must be sufficiently large such as to avoid unwanted interference. In this example, we have taken $T_{\text {recur }}=10$ days. By linearity, the basin response to the forcing in Eq.(2.27) will be the superposition of the responses to the individual periodic forcings as calculated by our model.

Now let us consider two wind events I and II, that impose a spatially uniform wind forcing of $3 \mathrm{~N} \mathrm{~m}^{-2}$ onto our reference basin in the along-basin direction (parameter values in Table 2.1). According to standard empirical friction laws (Wu, 1982), this corresponds to a wind speed of about $30 \mathrm{~m} \mathrm{~s}^{-1}$ (at $10 \mathrm{~m}$ height). The two wind events differ in their duration: wind event I lasts 10.3 hours, wind event II lasts 6 hours. Regarding spin-up and spin-down of the forcing, we apply a smooth transition taking 1.2 hours for both wind events (see grey curves in Fig.2.10bd).

The elevation at the evaluation point, as a function of time, shows quite different responses for the two wind events (see black curves in Fig.2.10bd). The strongest sloshing is observed after wind event II, whereas wind event I produces relatively little oscillations. This is as expected from comparing the 

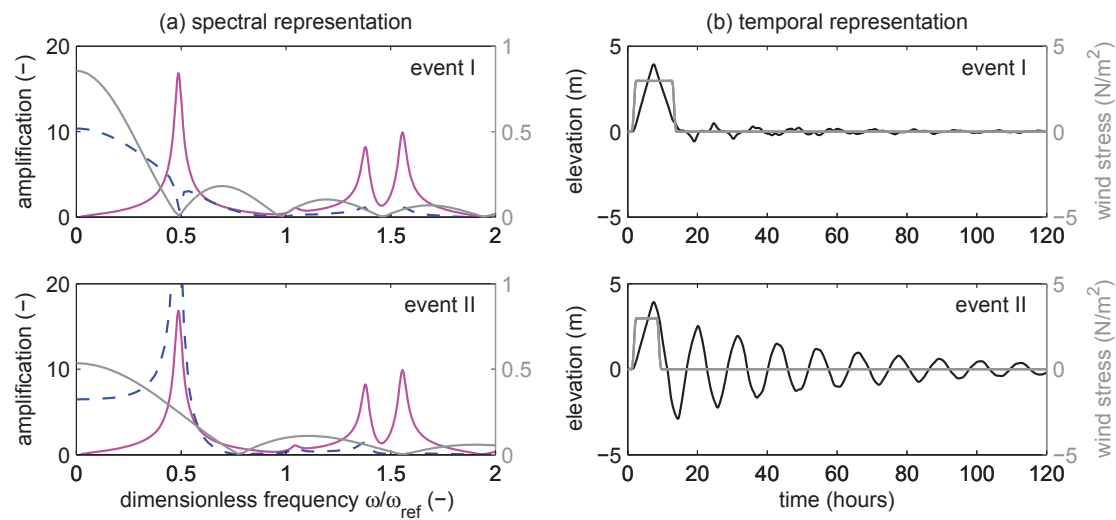

Figure 2.10: Response of surface elevation at evaluation point for two wind events that differ in duration: 10.3 hours for event I (top), 6 hours for event II (bottom). The plots on the left show the spectral representation of the wind (grey) as well as the amplification (pink), and the actual response (dashed). The plots on the right show the temporal representation of both forcing (grey) and response (black). Note that the grey curve represents the wind forcing as it is actually simulated using a superposition of Fourier modes. Parameter values as in Table 2.1, spatially uniform wind.

wind spectrum with the spectral response of the basin (see Fig.2.10ac). It is the product of these two quantities that gives the spectral response to the wind event under consideration (blue curve). Indeed, the resonant frequency $L / \lambda=0.49$ is not contained in the wind spectrum of event I, whereas it is present in event II.

\subsection{Conclusions}

We have developed an idealised process-based model to systematically investigate the resonance properties of closed rectangular rotating basins of uniform depth, subject to space- and time-dependent wind forcing. We focus on the spectral response of the surface elevation at an evaluation point midway one of the cross-basin boundaries.

Regarding the resonance peaks, we conclude that the spatial structure of the wind forcing matters. For example, without rotation, a spatially uniform wind produces the classical resonance peaks at $L / \lambda=\frac{1}{2}, \frac{3}{2}, \cdots$, whereas divergent wind also gives peaks at $L / \lambda=1,2, \cdots$. Including rotation shifts these peaks.

Next, because the cross-wind basin dimension $B$ is not small, cross-wind dynamics produces peaks at frequencies significantly lower than obtained by Ponte (2010). These cross-wind dynamics can be triggered by several mechanisms. Firstly, a wind forcing with nonzero curl produces cross-wind variations in elevation and thus cross-basin flow and oscillations that may be resonant. Secondly, the Coriolis acceleration of the along-wind flow also produces cross-basin oscillations. As discussed above, this along-wind pattern depends on the spatial 
pattern of the wind forcing, so these rotation-induced peaks will be different for spatially uniform wind and divergent wind.

In each of the above cases, the main effect of increasing friction is a lowering of the peaks. Finally, we have illustrated how the spectral response analysed above manifests itself in the response to a single wind event, and particularly how excitation of resonant frequencies produces sloshing in the basin. Extending this model approach with respect to geometry (realistic topography and coastlines) and atmospheric forcing is essential before making a detailed comparison with observations.

Acknowledgement: This work is partly funded by the Chinese Scholarship Council and partly by the research programme 'Impact of climate change and human intervention on hydrodynamics and environmental conditions in the Ems-Dollart estuary: an integrated data-modelling approach'. The latter project is financed by the Bundesministerium fur Bildung und Forschung (BMBF) and by the Netherlands Organization for Scientific Research (NWO), as part of the international Wadden Sea programme (GEORISK project). We thank one anonymous reviewer for his/her comments. 


\section{Appendix}

\section{A Expressions for flow and problem for $N$}

\section{A.1 Vertical profiles from horizontal momentum equations}

Here we present the details of the vertical structure of the flow First we define rotating flow components according to $q^{ \pm}=u \pm i v$ with complex amplitudes $Q^{ \pm}$, such that $U=\left(Q^{+}+Q^{-}\right) / 2$ and $V=\left(Q^{+}-Q^{-}\right) /(2 i)$. From Eqs.(2.1)-(2.2), the differential equation for the complex amplitude $Q^{ \pm}$is given by

$$
\frac{\mathrm{d}^{2} Q^{ \pm}}{\mathrm{d} z^{2}}-\lambda^{ \pm 2} Q^{ \pm}=g \mathcal{L}^{ \pm} N
$$

with complex operators $\mathcal{L}^{ \pm}=\partial / \partial x \pm i \partial / \partial y$. From Eqs.(2.4)-(2.5), the boundary conditions are given by

$$
K \frac{\mathrm{d} Q^{ \pm}}{\mathrm{d} z}=T^{ \pm} \quad \text { at } z=0, \quad \text { and } \quad K \frac{\mathrm{d} Q^{ \pm}}{\mathrm{d} z}=s Q^{ \pm} \quad \text { at } z=-h
$$

with rotating wind forcing amplitudes $T^{ \pm}=T^{(x)} \pm i T^{(y)}$ (wind stress divided by density). The two forcing terms in this nonhomogeneous differential problem imply that the rotating flow solution contains two contributions, proportional to the surface gradient and the wind stress, respectively:

$$
Q^{ \pm}(z)=Q_{\eta}^{ \pm}(z) \mathcal{L}^{ \pm} N+Q_{\mathrm{w}}^{ \pm}(z) T^{ \pm}
$$

The vertical structures read

$$
\begin{aligned}
Q_{\eta}^{ \pm}(z) & =\frac{g\left[\cosh \lambda^{+} z-\alpha_{\mathrm{c}}^{ \pm}\right]}{\alpha_{\mathrm{c}}^{ \pm} K \lambda^{ \pm 2}} \\
Q_{\mathrm{w}}^{ \pm}(z) & =\frac{\alpha_{\mathrm{c}}^{ \pm} \sinh \lambda^{ \pm} z+\alpha_{\mathrm{s}}^{ \pm} \cosh \lambda^{ \pm} z}{\alpha_{\mathrm{c}}^{ \pm} K \lambda^{ \pm}}
\end{aligned}
$$

with $\lambda^{ \pm 2}=-i(\omega \mp f) / K$ and $\alpha_{\mathrm{c}}^{ \pm}=\cosh \lambda^{ \pm} h+s^{-1} K \lambda^{ \pm} \sinh \lambda^{ \pm} h$ and $\alpha_{\mathrm{s}}^{ \pm}=\sinh \lambda^{ \pm} h+$ $s^{-1} K \lambda^{ \pm} \cosh \lambda^{ \pm} h$. The vertical integral is given by

$$
\left\langle Q^{ \pm}\right\rangle=\left\langle Q_{\eta}^{ \pm}\right\rangle \mathcal{L}^{ \pm} N+\left\langle Q_{\mathrm{w}}^{ \pm}\right\rangle T^{ \pm}
$$

with

$$
\begin{aligned}
\left\langle Q_{\eta}^{ \pm}\right\rangle & =\frac{g\left[\sinh \lambda^{ \pm} h-\alpha_{\mathrm{c}}^{ \pm} \lambda^{ \pm} h\right]}{\alpha_{\mathrm{c}}^{ \pm} K \lambda^{ \pm 3}}, \\
\left\langle Q_{\mathrm{w}}^{ \pm}\right\rangle & =\frac{\alpha_{\mathrm{c}}^{ \pm}\left[1-\cosh \lambda^{ \pm} h\right]+\alpha_{\mathrm{s}}^{ \pm} \sinh \lambda^{ \pm} h}{\alpha_{\mathrm{c}}^{ \pm} K \lambda^{ \pm 2}} .
\end{aligned}
$$

The two cases $\omega= \pm f$ require alternative expressions for either $Q^{+}$or $Q^{-}$. If $\omega=+f$ we must replace the $Q^{+}$-expressions in Eqs.(2.31)-(2.33); if $\omega=-f$ we must replace the $Q^{+}$-expressions. They must be replaced with

$$
Q_{\eta}^{ \pm}(z)=\frac{g h^{2}}{K}\left[\frac{1}{2}\left(\frac{z}{h}\right)^{2}-\frac{1}{2}-\frac{K}{s h}\right], \quad Q_{\mathrm{w}}^{ \pm}(z)=\frac{h}{K}\left[1+\left(\frac{z}{h}\right)+\frac{K}{s h}\right],
$$

and

$$
\left\langle Q_{\eta}^{ \pm}\right\rangle=-\frac{g h^{3}}{K}\left[\frac{1}{3}+\frac{K}{s h}\right], \quad\left\langle Q_{\mathrm{w}}^{ \pm}\right\rangle=\frac{h^{2}}{K}\left[\frac{1}{2}+\frac{K}{s h}\right] .
$$




\section{A.2 Elliptical problem for $N$}

Depth-integration of the continuity equation (2.3), with the aid of boundary conditions (2.4) gives, in terms of the complex amplitudes of surface elevation and the rotating velocity components.

$$
-i \omega N+\frac{\partial}{\partial x} \underbrace{\left(\frac{\left\langle Q^{+}\right\rangle+\left\langle Q^{-}\right\rangle}{2}\right)}_{\langle U\rangle}+\frac{\partial}{\partial y} \underbrace{\left(\frac{\left\langle Q^{+}\right\rangle-\left\langle Q^{-}\right\rangle}{2 i}\right)}_{\langle V\rangle}=0,
$$

Substitution of Eq.(2.30) gives the elliptical equation for $N$ presented in Eq.(2.11) of the main text. The corresponding coefficients are given by

$$
C_{1}=\frac{1}{2}\left[Q_{\eta}^{+}+Q_{\eta}^{-}\right], \quad C_{2}=\frac{1}{2 i}\left[Q_{\eta}^{+}-Q_{\eta}^{-}\right],
$$

The boundary conditions presented in Eqs.(2.13)-(2.14) of the main text follow from depth-integration of the momentum equations (2.1)-(2.2). The coefficients in there are given by $\gamma=\left\langle C_{2}\right\rangle /\left\langle C_{1}\right\rangle$ and

$$
R_{1}=\frac{1}{2\left\langle C_{1}\right\rangle}\left[Q_{\mathrm{w}}^{+} T^{+}+Q_{\mathrm{w}}^{-} T^{-}\right], \quad R_{2}=\frac{1}{2 i\left\langle C_{1}\right\rangle}\left[Q_{\mathrm{w}}^{+} T^{+}-Q_{\mathrm{w}}^{-} T^{-}\right] .
$$

\section{A.3 Vertical flow velocity}

The vertical flow velocity amplitudes at any depth $z$ are given by

$$
W(z)=-\left\lfloor C_{1}\right\rfloor\left(\frac{\partial^{2} N}{\partial x^{2}}+\frac{\partial^{2} N}{\partial y^{2}}\right)-\left\langle C_{1}\right\rangle\left(\left\lfloor\frac{\partial R_{1}}{\partial x}\right\rfloor+\left\lfloor\frac{\partial R_{2}}{\partial y}\right\rfloor\right),
$$

where floor brackets indicate integration from bottom to $z$, i.e. $\lfloor\cdot\rfloor=\int_{-h}^{z} \cdot \mathrm{d} z$. This expression can be simplified further by using the differential equation (2.11) for $N$ to eliminate the Laplacian of $N$.

\section{B Details of the collocation method}

\section{B.1 Expressions for $\phi^{\text {unif }}, \phi^{\text {div }}$ and $\phi^{\text {curl }}$}

Our solution method uses functions that homogenize the differential equation (2.11) and either the along-basin or cross-basin boundary conditions in Eqs.(2.13)-(2.14). For the three different parts of the wind field, these functions are given by

$$
\begin{aligned}
\phi^{\mathrm{unif}}(y) & =-\frac{\left[\left\langle Q_{\mathrm{w}}^{+}\right\rangle-\left\langle Q_{\mathrm{w}}^{-}\right\rangle\right] \hat{T}}{2 i\left\langle C_{1}\right\rangle k}\left[\sin k y-\chi_{k}^{-} \cos k y\right], \\
\phi^{\mathrm{div}}(x) & =\frac{\left[\left\langle Q_{\mathrm{w}}^{+}\right\rangle+\left\langle Q_{\mathrm{w}}^{-}\right\rangle\right] \hat{T}}{4\left\langle C_{1}\right\rangle k}\left[\sin k x+\xi_{k}^{+} \cos k x-\frac{2}{k L}\right], \\
\phi^{\text {curl }}(y) & =\frac{\left[\left\langle Q_{\mathrm{w}}^{+}\right\rangle-\left\langle Q_{\mathrm{w}}^{-}\right\rangle\right] \hat{T}}{4 i\left\langle C_{1}\right\rangle k}\left[\sin k y+\chi_{k}^{+} \cos k y-\frac{2}{k B}\right],
\end{aligned}
$$

with $k$ as defined in Eq. (2.11) and $\chi_{k}^{ \pm}=(1 \pm \cos k B) / \sin k B$ and $\xi_{k}^{ \pm}=(1 \pm \cos k L) / \sin k L$. 


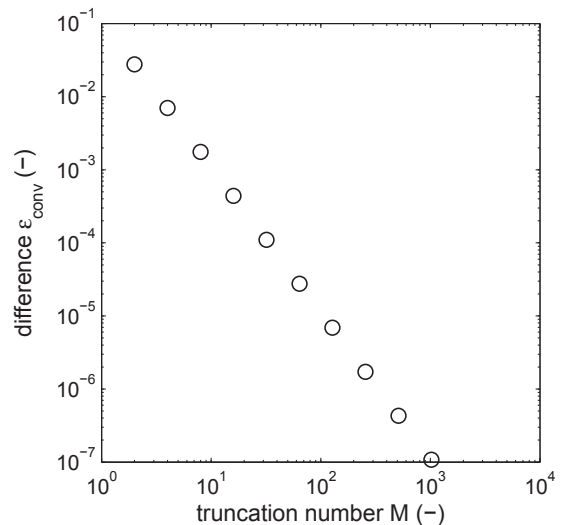

Figure 2.11: Difference $\epsilon_{\text {conv }}$ between two possible solution techniques available for the case with spatially uniform wind, as defined in Eq.(2.46). $M$ is the truncation number used for the solution with along-basin modes. Parameter values as in Table 2.1.

\section{B.2 Convergence test for spatially uniform wind}

As already pointed out in $\S 2.3$, the case of spatially uniform wind can be solved in two ways. The first is by combining along-basin modes with collocation points at the cross-basin boundaries (as in Fig.2.3a and in the main text); the second by combining cross-basin modes and collocation points at the along-basin boundaries (Fig.2.3b). The latter choice requires an alternative particular solution; Eq.(2.42) should be replaced with

$$
\tilde{\phi}^{\text {unif }}(x)=-\frac{\left[\left\langle Q_{\mathrm{w}}^{+}\right\rangle+\left\langle Q_{\mathrm{w}}^{-}\right\rangle\right] \hat{T}}{2\left\langle C_{1}\right\rangle k}\left[\sin k x-\xi_{k}^{-} \cos k x\right] .
$$

The symmetry property mentioned above allows us to perform a convergence test by intercomparing the two solutions for different truncation numbers. Using superscripts to denote the two ways of solution, we calculate the difference

$$
\epsilon_{\mathrm{conv}}=\frac{1}{M_{\mathrm{colloc}}} \sum_{m}\left|N^{\mathrm{unif}}\left(x_{m}, y_{m}\right)^{(1)}-N^{\mathrm{unif}}\left(x_{m}, y_{m}\right)^{(2)}\right|,
$$

averaging over the combined set of $M_{\text {colloc }}$ collocation points $\left(x_{m}, y_{m}\right)$ along the boundaries (see circles in Fig.2.3ab). In doing so, we make sure that the collocation spacing for both solutions is equal, i.e. $B / M$ equals $L / \tilde{M}$. The result of doing this for different values of the truncation number $M$ is shown in Fig.2.11. The figure displays second order convergence (Boyd, 2000).

\section{B.3 Channel modes: Kelvin and Poincaré waves}

Here we present expressions for the surface elevation amplitudes of the along-basin and cross-basin channel modes, used in $§ 2.3 .2$.

First, along-basin channel modes are wave solutions in an infinitely long channel aligned with the $x$-axis, and of width $B$. This means that these modes satisfy the homogenised elliptic equation (2.11) for $N$ as well as the homogenised cross-basin boundary conditions in Eq.(2.14), while having a harmonic along-basin structure $\exp \left(i \kappa_{m} x\right)$ 
with complex wave number $\kappa_{m}$. We thus identify infinitely many modes, characterised by

$$
\kappa_{0}^{2}=\frac{k^{2}}{1+\gamma^{2}}, \quad \kappa_{m}^{2}=k^{2}-\left(\frac{m \pi}{B}\right)^{2}, \quad(m=1,2, \cdots) .
$$

For each $m$, these quadratic relationships yield two wave numbers. We thus distinguish two families of modes. The modes having a wave number with a positive imaginary part $\Im\left\{\kappa_{m}\right\}>0$ propagate and/or decay exponentially in the positive $x$-direction are termed positive modes and denoted with a superscript $\oplus$. The modes having a wave number with a negative imaginary part $\Im\left\{\kappa_{m}\right\}<0$ propagate and/or decay exponentially in the negative $x$-direction are termed negative modes and denoted with a superscript $\ominus$.

Within each of these two families of modes, we identify a Kelvin wave (corresponding to $m=0)$ and infinitely many Poincaré waves $(m=1,2, \cdots)$. The spatial structure of the Kelvin mode is given by

$$
N_{0}^{\oplus}(x, y)=\exp \left(\frac{-y}{R_{\mathrm{def}}}\right) \exp \left(i \kappa_{0}^{\oplus} x\right), \quad R_{\mathrm{def}}=\sqrt{\frac{1+\gamma^{2}}{\gamma^{2} k^{2}}},
$$

where, due to vertical friction, the Rossby deformation radius $R_{\text {def }}$ is now a complex quantity. The spatial structure of the Poincaré modes is given by:

$$
N_{m}^{\oplus}(x, y)=\left[\cos \left(\frac{m \pi y}{B}\right)-\frac{\gamma i k B}{m \pi} \sin \left(\frac{m \pi y}{B}\right)\right] \exp \left(i \kappa_{m}^{\oplus} x\right), \quad(m=1,2, \cdots) .
$$

Expressions for the negative Kelvin and Poincaré modes follow similarly.

Analogously, the cross-basin channel modes are wave solutions in an infinitely long channel aligned with the $y$-axis, and of width $L$. This means that these modes satisfy the homogenised elliptic equation (2.11) for $N$ as well as the homogenised along-basin boundary conditions in Eq.(2.13), while having an exponential cross-basin structure $\exp (i \tilde{\kappa} y)$ with wave number $\tilde{\kappa}$. The two families of cross-basin channel modes follow from the along-basin modes by replacing $B$ with $L$ and considering a rotated coordinate system.

\section{B.4 Equilibrium response to steady wind forcing $(\omega=0)$}

Here, we will solve the equilibrium response to a steady wind forcing $(\omega=0)$. The free surface elevation amplitude satisfies a Poission problem:

$$
\frac{\partial^{2} N}{\partial x^{2}}+\frac{\partial^{2} N}{\partial y^{2}}=-\left[\frac{\partial\left\langle R_{1}\right\rangle}{\partial x}+\frac{\partial\left\langle R_{2}\right\rangle}{\partial y}\right]
$$

with boundary conditions as in Eqs.(2.13)-(2.14).

For the solution due to the spatially uniform part of the wind field, the solution is a linear profile sloping in the along-basin direction only:

$$
N^{\mathrm{unif}}=-\Lambda\left(x-\frac{L}{2}\right), \quad \Lambda=\frac{\left\langle Q_{\mathrm{w}}^{+}+Q_{\mathrm{w}}^{-}\right\rangle}{\left\langle Q_{\eta}^{+}+Q_{\eta}^{-}\right\rangle} \hat{T} .
$$

The solution due to the divergent part of the wind field is solved by means of a collocation technique largely similar to the one presented in $\S 2.3 .2$. However, there are three notable differences: $(i)$ the particular solution $\phi^{\text {div }}$ has a different form, $(i i)$ the Kelvin modes must be replaced with a linearly sloping function $\psi^{\text {div }}$ and a constant 
function, (iii) the Poincaré modes are as in Appendix 2.B.3 but now with $k=0$, and $(i v)$ the boundary condition at one of the collocation points must be replaced by the overall statement that water is conserved. We write

$$
\begin{aligned}
N^{\mathrm{div}}= & \phi^{\mathrm{div}}(x)+a_{0}^{\mathrm{sl}} \psi^{\mathrm{div}}(x, y)+a_{0}^{\text {const }}+ \\
& \sum_{m=1}^{\tilde{M}} a_{m}^{\oplus} \tilde{N}_{m}^{\oplus}(x, y)+\sum_{m=1}^{\tilde{M}} a_{m}^{\ominus} \tilde{N}_{m}^{\ominus}(x, y),
\end{aligned}
$$

with $\phi^{\text {div }}(x)=-\frac{1}{2} \Lambda\left(x-\frac{1}{2} L\right)^{2}, \psi^{\text {div }}(x, y)=\gamma x-y$ and associated coefficients $a_{0}^{\text {sl }}$ and $a_{0}^{\text {const }}$. We always use an even number for $\tilde{M}$, and we select the collocation point midway one of the collocation boundaries for the conservation condition. A second solution is then obtained by doing the same, but now selection the collocation point midway the other boundary. Averaging the two finally leads to a solution that is symmetric with respect to the collocation method.

For the solution due to curl part of the wind field, a similar approach is followed:

$$
\begin{aligned}
N^{\text {curl }}= & \phi^{\text {curl }}(y)+b_{0}^{\text {sl }} \psi^{\text {curl }}(x, y)+b_{0}^{\text {const }}+ \\
& \sum_{m=1}^{M} b_{m}^{\oplus} N_{m}^{\oplus}(x, y)+\sum_{m=1}^{M} b_{m}^{\ominus} N_{m}^{\ominus}(x, y),
\end{aligned}
$$

with $\phi^{\text {curl }}(x)=-\frac{1}{2} \Lambda\left(y-\frac{1}{2} B\right)^{2}, \psi^{\text {curl }}(x, y)=x+\gamma y$ and associated coefficients $b_{0}^{\text {sl }}$ and $b_{0}^{\text {const }}$.

\section{C Details of the expansion in $f / \omega$}

At first order, i.e. at $\mathcal{O}(\epsilon)$ with $\epsilon=f / \omega$, the elevation amplitude and flow field are given by

$$
\begin{aligned}
\frac{N_{1}}{\hat{N}}= & i \sum_{n \text { even }} d_{n} \cos \left(\alpha_{n} x\right) F_{\tilde{\beta}_{n}}^{-}(y)+i a \sum_{n \text { odd }} e_{n} \cos \left(\alpha_{n} x\right) F_{\tilde{\beta}_{n}}^{-}(y) \\
& -i b\left\{\frac{2}{k_{0} B}+\sum_{m \text { odd }} \sum_{n \text { even }}\left(j_{m n} F_{\tilde{\alpha}_{n}}^{+}(x) \cos \beta_{n} y+k_{m n} \cos \left(\alpha_{n} x\right) F_{\tilde{\beta}_{n}}^{+}(y)\right)\right\} \\
\frac{U_{1}}{\hat{U}}= & -\sum_{n \text { even }} d_{n} \frac{\alpha_{n}}{k_{0}} \sin \left(\alpha_{n} x\right) F_{\tilde{\beta}_{n}}^{-}(y)-a \sum_{n \text { odd }} e_{n} \frac{\alpha_{n}}{k_{0}} \sin \left(\alpha_{n} x\right) F_{\tilde{\beta}_{n}}^{-}(y) \\
& +b \sum_{m \text { odd }}\left(\sum_{n \text { even }} j_{m n} \frac{\tilde{\alpha}_{n}}{k_{0}} I_{\tilde{\alpha}_{n} \alpha_{m}}(x) \cos \beta_{n} y+k_{m n} \frac{\alpha_{n}}{k_{0}} \sin \left(\alpha_{n} x\right) F_{\tilde{\beta}_{n}}^{+}(y)\right), \\
\frac{V_{1}}{\hat{U}}= & -\sum_{n \text { even }} d_{n} \frac{\tilde{\beta}_{n}}{k_{0}} \cos \left(\alpha_{n} x\right) G_{\tilde{\beta}_{n}}^{-}(y)-a \sum_{n \text { odd }} e_{n} \frac{\tilde{\beta}_{n}}{k_{0}} \cos \left(\alpha_{n} x\right) G_{\tilde{\beta}_{n}}^{-}(y) \\
& +b\left\{\sum_{m \text { odd }} \sum_{n \text { even }}\left(j_{m n} \frac{\beta_{n}}{k_{0}} F_{\tilde{\alpha}_{n}}^{+}(x) \sin \beta_{n} y-k_{m n} \frac{\tilde{\beta}_{n}}{k_{0}} \cos \left(\alpha_{n} x\right) H_{\tilde{\beta}_{n} \beta_{m}}^{+}(y)\right)\right\}
\end{aligned}
$$


respectively. Here, we have introduced wave numbers $\alpha_{n}=n \pi / L$ and $\tilde{\beta}_{n}$ satisfying $\alpha_{n}^{2}+\tilde{\beta}_{n}^{2}=k_{0}^{2}$. Next, the coefficients $d_{n}$ and $e_{n}$ are given by

$$
d_{0}=\frac{2 \xi_{k_{0} L}^{-}}{k_{0} L}-1, \quad d_{n}=\frac{2 k_{0} L \xi_{k_{0} L}^{-}}{\left(\tilde{\beta}_{n} L\right)^{2}},
$$

for $n$ nonzero and even, as well as

$$
e_{n}=\frac{8 \sin k_{0} L}{\left(\alpha_{n} L\right)^{2}}+\frac{4 k_{0} L \xi_{k_{0} L}^{+}}{\left(\tilde{\beta}_{n} L\right)^{2}}
$$

for $n$ odd. For $m$ odd and $n$ even, we have

$$
\begin{aligned}
& j_{m 0}=c_{m} \frac{\beta_{m}}{k_{0}} \frac{2 \xi_{\tilde{\alpha}_{m} L}^{-}}{m \pi}, \quad j_{m n}=c_{m} \frac{\beta_{m}}{\tilde{\alpha}_{n}} \frac{4 \xi_{\tilde{\alpha}_{m} L}^{-} m}{\pi\left(m^{2}-n^{2}\right)}, \\
& k_{m 0}=c_{m} \frac{\tilde{\alpha}_{m}}{k_{0}}\left[1-\frac{2 \xi_{\alpha_{n} L}^{-}}{\tilde{\alpha}_{m} L}\right], \quad k_{m n}=c_{m} \frac{\tilde{\alpha}_{m}}{\tilde{\beta}_{n}} \frac{4 \xi_{\tilde{\alpha}_{m} L}^{-\tilde{\alpha}_{m} L}}{\left(\alpha_{n} L\right)^{2}-\left(\tilde{\alpha}_{m} L\right)^{2}} .
\end{aligned}
$$

As new functions, we have used $H_{\tilde{\beta}_{n} \beta_{m}}^{+}(y)=1-\cos \beta_{m} y-G_{\tilde{\beta}_{n}}^{+}(y)$ and $I_{\tilde{\alpha}_{n} \tilde{\alpha}_{m}}(x)=$ $G_{\tilde{\alpha}_{n}}^{+}(x)-1-F_{\tilde{\alpha}_{m}}^{-}(x) / \xi_{\tilde{\alpha}_{m} L}$. 


\title{
Chapter 3
}

\section{Response of large-scale coastal basins to wind forcing: influence of topography ${ }^{\star}$}

\begin{abstract}
We present an idealised process-based model to study the influence of topographic variations on the frequency response of large-scale coastal basins subject to time-periodic wind forcing. Coastal basins are represented by a semi-enclosed rectangular inner region forced by wind. It is connected to an outer region (represented as an infinitely long channel) without wind forcing, which allows waves to freely propagate outward. The model solves the three-dimensional linearised shallow water equations on the $f$ plane, forced by a spatially uniform wind field that has an arbitrary angle with respect to the along-basin direction. The surface elevation amplitudes, and hence the vertical profiles of the velocity, are obtained using the Finite Element Method (FEM), extended to account for the connection to the outer region. The results are then evaluated in terms of the elevation amplitude averaged over the basin's landward end, as a function of the wind forcing frequency. In general, the results point out that adding topographic elements in the inner region (such as a topographic step, a linearly sloping bed or a parabolic cross-basin profile), causes the resonance peaks to shift in the frequency domain, through their effect on local wave speed. The Coriolis effect causes the resonance peaks associated with cross-basin modes (which without rotation only appear in the response to cross-basin wind) to emerge also in the response to along-basin wind and vice versa.
\end{abstract}

\subsection{Introduction}

Wind driven set-up is the main contribution to extreme high water events, which may threaten coastal safety. This is particularly so when the combined characteristics of the wind forcing and the basin trigger resonance (Abraham, 1960). A typical example is typhoon Winnie at the Korean coast of the Yellow Sea in 1997. The unusually strong and extensive coastal flooding was partly caused by resonant coupling of the Yellow Sea and the predominant period of the forcing (Moon et al, 2003).

Importantly, the resonance properties of coastal basins can be affected by large-scale topographic elements. For example, shoals may protect the coast (Hanley et al, 2014), while on the other hand, a longshore bar can generate storm wave resonance under certain circumstances (Büsching, 2003). Moreover, wind

\footnotetext{
${ }^{\star}$ This chapter is now under review. Chen, W.L., Roos, P.C., Schuttelaars, H.M., Kumar, M., Zitman, T.J. and Hulscher, S.J.M.H. (2015, submitted). Response of large-scale coastal basins to wind forcing: influence of topography.
} 
blowing in different directions in a semi-enclosed basin may lead to significantly different responses (Breaker et al, 2010). For coastal safety, an overall practical goal is to be able to predict the wind driven water levels at any location in basins of arbitrary shape and size. This requires physical insight in the influence of large-scale topography on resonance properties of large-scale coastal basins. Below we will review the literature on this topic.

The influence of topography on surge response has been investigated in various site-specific studies using numerical models. For example, Chen et al (2008) suggested that the record-high storm surge of Hurricane Katrina (New Orleans, Louisiana) was caused by the interaction of the surge with the extremely shallow, ancient deltaic lobe of the Mississippi river. For this case, Irish et al (2008) found that a milder shelf slope would have led to a higher surge. Alternatively, Weaver and Slinn (2010) found that small-scale variations in nearshore bathymetry of about $20 \%$ produce smaller variations in storm surge at the shoreline (less than $5 \%$ ). They applied this to three study sites. Using a one-dimensional numerical model, Libicki and Bedford (1990) showed how westward traveling storms over Lake Erie (approaching shallower regions) produce higher surge levels than eastward traveling storms (approaching deeper regions). While these studies produce site-specific results, it is difficult to draw generic conclusions.

On the other hand, more generic studies focus on the frequency response of the systems to wind forcing. This is because a wind event can be seen as the superposition of periodic wind forcings at various frequencies $\omega$ (Craig, 1989). Assuming linear dynamics, also the response, i.e. the flow and elevation pattern, will be the superposition of the responses at these individual frequencies. Hence, the basin's response to a wind event is contained in its so-called frequency response. Proudman (1929) provided analytical solutions for the response in narrow closed basins with a single topographic step. Alternatively, Ponte (2010) investigated the response of large-scale, elongated closed basins with a parabolic cross-basin topography to along-basin wind forcing. Recently, Chen et al (2015) extended this approach to closed basins with comparable length and width, but restricted to uniform depth. Other studies focused on the eigenmodes of, e.g., closed basins with uniform depth (Rao, 1966) or small-scale semi-enclosed basins with topography (Wilson, 1972; Sobey, 2006; Rabinovich, 2009).

The goal of the present study is to investigate the influence of large-scale topography on the wind-driven frequency response of large-scale coastal basins, measured in terms of the set-up at the coast, and paying particular attention to the role of the Coriolis effect and wind angle.

To achieve this goal, we have developed an idealised three dimensional process-based model of a semi-enclosed rectangular rotating coastal basin subject to periodic wind forcing. The vertical profile of the flow field is resolved fully analytically, and expressed in terms of the free surface elevation. In turn, the spatial pattern of free surface elevation amplitudes follows from solving an elliptic problem using the Finite Element Method (FEM), extended to account for the connection of the coastal basin to the outer sea.

With this model, the frequency response of a coastal basin subject to spatially uniform periodic wind is investigated, both without and with rotation. We 
will consider a spatially uniform wind field with directions ranging from alongbasin to cross-basin. The influence of topography on the frequency response is then investigated by systematically adding topographic details, expressing along-basin and cross-basin variations.

This paper is organised as follows. In section 3.2, we present the model. Next, section 3.3 contains the solution method. The model results, showing the frequency responses for the various topographic elements introduced above, as well as the discussion are presented in section 3.4. Finally, section 3.5 contains the conclusions.

\subsection{Model formulation}

\subsubsection{Geometry}

Our model geometry consists of two parts: an inner region and an outer region (see Figure 3.1).

- The inner region, of length $L$ and uniform width $B$, represents a rectangular semi-enclosed coastal basin. This is where the wind forcing takes place, and where topographic elements will be added.

- The outer region, also of uniform width $B$, represents an outer sea. It stretches to infinity and experiences no wind forcing. By including the outer region, we allow wave energy to travel away from the inner region without reflecting at the interface with the outer region.

A ramp-up zone of length $L_{\text {ramp }}$, part of the inner region, serves as a transition zone where the wind gradually increases from no wind in the outer region to a spatially uniform wind in the core of the inner region. This transition is described by a so-called ramp-up function $\mu(x)$, to be detailed further below in $\S 3.2 .2$. Without ramp-up zone, a discontinuity would occur in the wind field, which would produce unrealistic model results.

The along-basin and cross-basin coordinates are denoted by $x$ and $y$, respectively, such that the closed boundaries are located at $x=L$ and $y=0, B$ and the interface between outer and inner region at $x=0$. The vertical coordinate $z$ points upward, with $z=\eta_{j}(x, y, t)$ denoting the free surface elevation with respect to the undisturbed water level $z=0$. The subscript $j=0$ represents the outer region, the inner region is labeled with $j=1$. The bottom topography is assumed to be spatially uniform in the outer region, and is denoted by $z=-h_{0}$. Over the inner region, the topography is allowed to vary, i.e. $z=-h_{1}(x, y)$. We will consider the following typical inner basin topographies (Figure 3.1):

- uniform depth, which serves as a reference case (type ' 1 ' in Fig.3.1). Note that Chen et al (2015) also considered a uniform depth, but in a closed rather than a semi-enclosed basin.

- along-basin variations, such as a topographic step at $x=x_{\text {step. }}$. Such a step divides the inner region into two subcompartments: an offshore part 
with the same uniform depth $h_{0}$ as the outer region, and a coastal part with depth $h_{\mathrm{c}}$. We will consider situations with a shallower coastal part ( $h_{\mathrm{c}}<h_{0}$, typical for many basins such as the Gulf of California and the Adriatic Sea) as well as with a deeper coastal part $\left(h_{\mathrm{c}}>h_{0}\right.$, as e.g. in the Norwegian trench). The topographic step can be both abrupt (type '2') and smoothened (type ' 3 '). In the latter case, the depth varies gradually from $h_{0}$ to $h_{\mathrm{c}}$ in a region of length $L_{\text {slope }}$ centered around $x=x_{\text {step }}$ (precise shape to be detailed in §3.4.4). Finally, we will also consider a linear profile from $h_{0}$ at $x=L_{\text {ramp }}$ to $h_{\mathrm{c}}$ at $x=L$ (type ' 4 ').

- cross-basin variations, such as a parabolic cross-basin profile with a smooth transition over a length $L_{\text {slope }}$ to the spatially uniform depth $h_{0}$ in the outer region and ramp-up zone (type ' 5 '). Most natural basins are deepest along their centerlines (as for the Gulf of California; see Ponte et al, 2012). This profile will be detailed in $§ 3.4 .6$ and Fig.3.8.

\subsubsection{Hydrodynamics}

Let $\boldsymbol{u}_{j}=\left(u_{j}, v_{j}, w_{j}\right)$ represent the flow velocity vector, with components $u_{j}, v_{j}$ and $w_{j}$ in the $x, y$ and $z$-direction, respectively $(j=0,1)$. Assuming that the vertical displacement of the free surface is small compared to the water depth, conservation of momentum and mass is expressed by the three-dimensional linearised shallow water equations on the $f$ plane according to

$$
\begin{aligned}
\frac{\partial u_{j}}{\partial t}-f v_{j} & =-g \frac{\partial \eta_{j}}{\partial x}+K \frac{\partial^{2} u_{j}}{\partial z^{2}}, \\
\frac{\partial v_{j}}{\partial t}+f u_{j} & =-g \frac{\partial \eta_{j}}{\partial y}+K \frac{\partial^{2} v_{j}}{\partial z^{2}}, \\
\frac{\partial u_{j}}{\partial x}+\frac{\partial v_{j}}{\partial y}+\frac{\partial w_{j}}{\partial z} & =0
\end{aligned}
$$

Here, $f=2 \Omega \sin \vartheta$ is the Coriolis parameter (with $\Omega=7.292 \times 10^{-5} \mathrm{rad} \mathrm{s}^{-1}$ the angular frequency of the Earth's rotation and $\vartheta$ the latitude), $g=9.81 \mathrm{~m} \mathrm{~s}^{-2}$ the gravitational acceleration. Turbulence is represented using a spatially uniform vertical eddy viscosity $K$, combined with a partial slip condition at the bed. Horizontal mixing of momentum is neglected. The above linearisation further assumes that the effect of the advective terms around topographic elements can be neglected.

The kinematic and dynamic boundary conditions at the surface and bottom read, in linearised form:

$$
\begin{array}{ll}
w_{j}=\frac{\partial \eta_{j}}{\partial t}, \quad K\left(\frac{\partial u_{j}}{\partial z}, \frac{\partial v_{j}}{\partial z}\right)=\frac{\left(\tau_{\mathrm{w}}^{(x)}, \tau_{\mathrm{w}}^{(y)}\right)}{\rho} \quad \text { at } z=0, \\
w_{j}=0, \quad K\left(\frac{\partial u_{j}}{\partial z}, \frac{\partial v_{j}}{\partial z}\right)=s\left(u_{j}, v_{j}\right) \quad \text { at } z=-h_{j} .
\end{array}
$$




\section{(a) top view}

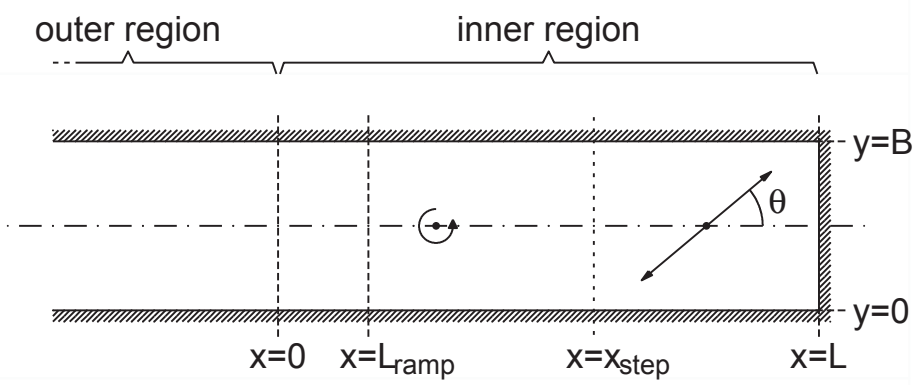

\section{(b) side view}

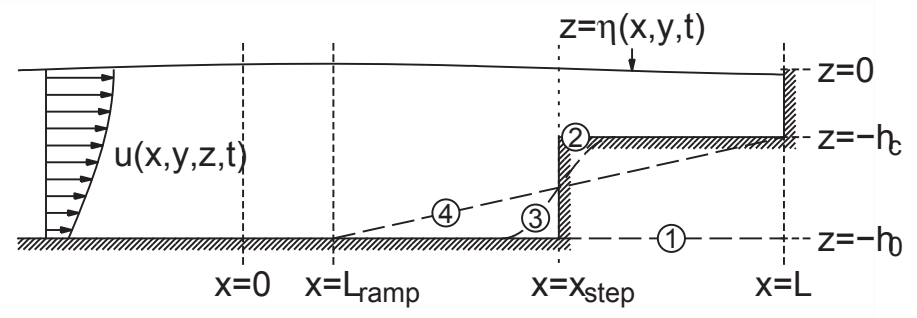

(c) ramp-up function

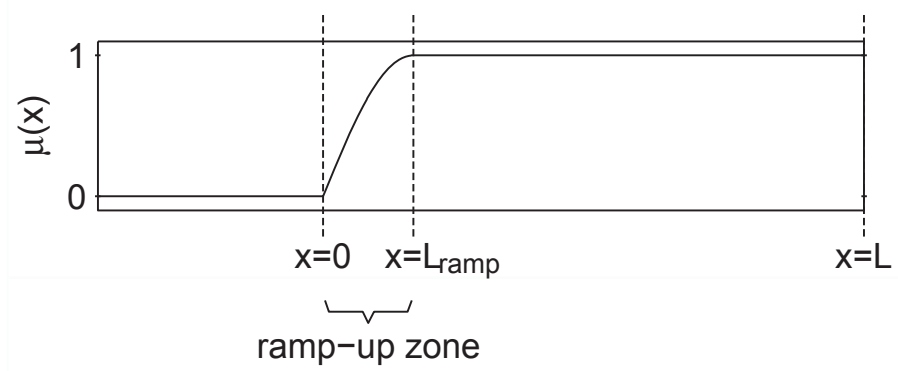

Figure 3.1: Definition sketch of the model geometry of a rectangular rotating basin, subject to periodic wind forcing, which makes an angle $\theta$ with the along-basin direction. (a) Top view, showing the outer region, ramp-up region and inner region. (b) Side view in along-basin direction displaying the free surface elevation as well as vertical profile of the along-basin component of the three-dimensional flow field. The topography illustrated here is that of an abrupt step (type 2 , here with $h_{\mathrm{c}}<h_{0}$ ), located at $x=x_{\text {step }}$. Dashed lines indicate three alternative topographies: uniform depth (type 1), smoothened step (type 3) and linear profile (type 4). For type 5, see Figure 3.8. (c) Ramp-up function $\mu(x)$ used to describe the transition from no wind in the outer region to spatially uniform wind conditions in the core of the inner region. 
The linearisation procedure implies that the free surface condition in Eq.(3.4) is imposed at $z=0$ instead of at $z=\eta$. Furthermore, $\left(\tau_{\mathrm{w}}^{(x)}, \tau_{\mathrm{w}}^{(y)}\right)$ is the wind stress vector and $\rho$ the density of water. Assuming a frequency $\omega$ and a wind angle $\theta$ with respect to the along-basin direction, we write

$$
\frac{\left(\tau_{\mathrm{w}}^{(x)}, \tau_{\mathrm{w}}^{(y)}\right)}{\rho}=\mu(x) \hat{T}(\cos \theta, \sin \theta) \cos \omega t .
$$

Here, the constant $\hat{T}$ is the amplitude of the wind stress divided by the water density. The ramp-up function $\mu(x)$ introduced earlier, as sketched in Fig.3.1, is specified in Appendix 3.A.

In Eq.(3.5), we have introduced a constant resistance parameter $s$, its value usually obtained from the analysis of field data. Two limiting cases are of interest. For large $s$, the bottom boundary condition effectively means no-slip, as used by e.g. Ponte et al (2012). On the other hand, $s=0$ corresponds to free-slip for which the flow becomes independent of $z$.

At the closed horizontal boundaries, we require zero normal transports, i.e.

$$
\left\langle u_{2}\right\rangle=0 \quad \text { at } x=L \quad \text { and } \quad\left\langle v_{j}\right\rangle=0 \quad \text { at } y=0, B,
$$

where $j=0,1$ and angle brackets denote vertical integration from bottom to surface, i.e. $\langle\cdot\rangle=\int_{-h_{j}}^{0} \cdot \mathrm{d} z$ (with the upper boundary $z=0$ arising from the linearisation).

At the interfaces between the adjacent regions, we require matching of surface elevation and normal transport:

$$
\eta_{0}=\eta_{1}, \quad\left\langle u_{0}\right\rangle=\left\langle u_{1}\right\rangle, \quad \text { at } x=0,
$$

where we note that the water depth is continuous across both interfaces (and equal to $\left.h_{0}\right)$. Finally, regarding the outer region, we allow no wave energy coming in from infinity (Sommerfeld type of condition). This means that the solution in the outer region will be written as a superposition of outward propagating waves (in the negative $x$-direction). Because we include the Coriolis effect, these waves include Kelvin and Poincaré waves.

\subsection{Solution method}

\subsubsection{Preliminary considerations: wind angle}

The linearity of our model implies that the solution for arbitrary wind angle $\theta$ can be written as a linear combination of the solutions $\eta_{\text {along }}$ for along-basin wind $\left(\theta=0^{\circ}\right)$ and $\eta_{\text {cross }}$ for cross-basin wind $\left(\theta=90^{\circ}\right)$ :

$$
\eta(x, y, t)=\eta_{\text {along }}(x, y, t) \cos \theta+\eta_{\text {cross }}(x, y, t) \sin \theta .
$$

This similarly applies to the solution of the flow components $u, v$ and $w$. In our description of the solution method, we will therefore distinguish between along-basin and cross-basin wind only. 


\subsubsection{Differential problem for surface elevation amplitude}

First we write the solution in the outer and inner regions as time-periodic functions according to

$$
\begin{aligned}
\eta_{j}(x, y, t) & =\Re\left\{N_{j}(x, y) \exp (-\mathrm{i} \omega t)\right\}, \\
u_{j}(x, y, z, t) & =\Re\left\{U_{j}(x, y, z) \exp (-\mathrm{i} \omega t)\right\},
\end{aligned}
$$

with $\Re$ denoting the real part and with complex amplitudes $N_{j}$ and $U_{j}(j=0,1)$. Similar expressions hold for $v_{j}$ and $w_{j}$, with complex amplitudes $V_{j}$ and $W_{j}$.

Next, we express the horizontal flow solution $U_{j}$ and $V_{j}$ in terms of surface slopes $\nabla N_{j}$ and wind stress. Details of this derivation can be found in Appendix 3.B. Substituting these expressions into the continuity equation and integrating from bottom to surface gives the following elliptic equation for $N$ :

$$
\nabla_{\mathrm{h}} \cdot\left[\left\langle\mathbf{D}_{j}\right\rangle \nabla_{\mathrm{h}} N_{j}\right]-\mathrm{i} \omega N_{j}=-\nabla_{\mathrm{h}} \cdot\left\langle\mathbf{r}_{j}\right\rangle,
$$

with horizontal nabla operator $\nabla_{\mathrm{h}}=(\partial / \partial x, \partial / \partial y)^{\mathrm{T}}$ as well as $2 \times 2$-matrix $\mathbf{D}_{j}$ and $2 \times 1$-vector $\mathbf{r}_{j}$, given by

$$
\mathbf{D}_{j}=\left[\begin{array}{cc}
C_{j}^{+} & C_{j}^{-} \\
-C_{j}^{-} & C_{j}^{+}
\end{array}\right], \quad \mathbf{r}_{j}=\left[\begin{array}{c}
R_{j}^{+} \\
R_{j}^{-}
\end{array}\right] .
$$

The coefficients $C_{j}^{+}$and $C_{j}^{-}$as well as the forcing terms $R_{j}^{+}$and $R_{j}^{-}$depend on topography and thus on $x$ and $y$; they are specified in Appendix 3.B.2.

The no normal transport conditions at the closed boundaries, as given by Eq. 3.7, imply

$$
\begin{gathered}
\left\langle C_{1}^{+}\right\rangle \frac{\partial N_{1}}{\partial x}+\left\langle C_{1}^{-}\right\rangle \frac{\partial N_{1}}{\partial y}=-\left\langle R_{1}^{+}\right\rangle \quad \text { at } x=L, \\
-\left\langle C_{j}^{-}\right\rangle \frac{\partial N_{j}}{\partial x}+\left\langle C_{j}^{+}\right\rangle \frac{\partial N_{j}}{\partial y}=-\left\langle R_{j}^{-}\right\rangle \quad \text { at } y=0, B .
\end{gathered}
$$

Finally, the vertical flow amplitude $W_{j}$ at any vertical position $z$ can be expressed in terms of the free surface elevation $N_{j}$ and wind forcing. This follows from vertical integration of the continuity equation (Appendix 2.A.3).

The matching conditions at $x=0$, as expressed in Eq.(3.8), now becomes

$$
\begin{gathered}
N_{0}=N_{1} \\
\left\langle C_{0}^{+}\right\rangle \frac{\partial N_{0}}{\partial x}+\left\langle C_{0}^{-}\right\rangle \frac{\partial N_{0}}{\partial y}=\left\langle C_{1}^{+}\right\rangle \frac{\partial N_{1}}{\partial x}+\left\langle C_{1}^{-}\right\rangle \frac{\partial N_{1}}{\partial y} .
\end{gathered}
$$

Due to the continuity of the wind forcing across the interface, the $R_{j}^{+}$-contributions to the matching condition in Eq.(3.17) cancel.

\subsubsection{Finite Element Method}

The problem in Eqs.(3.12)-(3.15) for the elevation amplitude $N_{j}$ is solved by applying the Finite Element Method (FEM, for the inner region), connected to a superposition of outward propagating waves (in the outer region). 
To this end, the inner region is discretised into a set of $P$ triangular elements. The solution is then written as

$$
N_{1}(x, y)=\sum_{p=0}^{P} N_{1, p} \phi_{p}(x, y),
$$

with coefficients $N_{1, p}$ and basis functions $\phi_{p}(x, y)$. To obtain the coefficients $N_{1, p}$, Eq.(3.12) is cast in weak form, and then integrated over the domain using test functions. Details can be found in Gockenbach (2006), and we also refer to Kumar et al (submitted), who developed a similar model to study tidal dynamics in estuaries.

The outer region deserves particular attention. Instead of applying a FEMgrid, the solution in the outer region is written as a truncated superposition of outward propagating waves. We thus write

$$
N_{0}(x, y)=\sum_{m=0}^{M} c_{0, m}^{\ominus} N_{m}^{\ominus}(y) \exp \left(\mathrm{i} k_{m}^{\ominus} x\right),
$$

with coefficients $c_{0, m}^{\ominus}$. As indicated by the $\ominus$ subscript, this expression involves modes propagating in the negative $x$-direction only. Their cross-basin structures $N_{m}^{\ominus}(y)$, corresponding to a Kelvin mode $(m=0)$ and Poincaré modes $(m=$ $1,2, \cdots)$, and the associated wave numbers $k_{m}^{\ominus}$ are specified in Appendix 3.E. Because the individual modes satisfy the closed boundary conditions at $y=0, B$ in the outer region, so does the superposition in Eq.(3.19).

To satisfy the matching conditions at the interface at $x=0$, we must connect the solutions (3.18) and (3.19) in the inner and outer region. This is done using a so-called collocation technique. We introduce a set of $M+1$ equidistant collocation points $(x, y)=\left(0, y_{m}\right)$ with $y_{m}=m B / M$ for $m=0,1, \cdots, M$. Eqs.(3.16)-(3.17) are then applied at each of these collocation points, where the left-hand side follows from Eq.(3.19) and the right-hand side follows from interpolation of the FEM-solution onto the collocation points.

This means that the FEM-model is extended to account for waves radiating away from the inner region. Indeed, the linear matrix system contains conditions for the $P$ coefficients $N_{1, p}$ in Eq.(3.18) as well as conditions for the $M+1$ coefficients $c_{0, m}^{\ominus}$ in Eq.(3.19). Effectively, the solution in the outer region and the matching conditions at $x=0$ pose a non-reflecting boundary condition for the inner region, even in the presence of the Coriolis effect.

Finally, in special cases, the solution can be obtained by quick (semi-)analytical methods, which can furthermore be used to test the FEM-model. We distinguish two cases:

- For $f=0$ and cross-basin wind $\left(\theta=90^{\circ}\right)$, the solution for uniform depth and the abrupt step topography can be found using a collocation technique also in the inner region. For the uniform depth case, the solutions in the ramp-up zone and the rest of the inner region are written as superpositions of a suitably chosen particular solution and two truncated families of $M+1$ 
Table 3.1: Overview of model parameters and their reference values.

\begin{tabular}{lcrl}
\hline Description & Symbol & Value & Unit \\
\hline Basin width & $B$ & $100,(200,400)$ & $\mathrm{km}$ \\
Total basin length & $L$ & 400 & $\mathrm{~km}$ \\
Length of coastal part & $L_{\mathrm{c}}$ & 100 & $\mathrm{~km}$ \\
Water depth (outer region) & $h_{0}$ & 100 & $\mathrm{~m}$ \\
Water depth (coastal part) & $h_{\mathrm{c}}$ & $15^{*}$ & $\mathrm{~m}$ \\
Latitude & $\vartheta$ & $0^{\dagger}, 50^{\ddagger}$ & ${ }^{\circ} \mathrm{N}$ \\
Vertical eddy viscosity & $K$ & 0.025 & $\mathrm{~m}^{2} \mathrm{~s}^{-1}$ \\
Resistance parameter & $s$ & $10^{-4}$ & $\mathrm{~m} \mathrm{~s}^{-1}$ \\
\hline
\end{tabular}

*varied from $10-1000 \mathrm{~m}$ for the topographic step (topography type 2) and from $10-100 \mathrm{~m}$ for the linear profile (type 4); ${ }^{\dagger}$ non-rotating basin; ${ }^{\ddagger}$ strongly rotating basin.

wave modes (one Kelvin mode and $M$ Poincaré modes) propagating in the positive and negative $x$-direction, respectively. The particular solution is chosen to homogenise the boundary conditions at $y=0, B$ (which are nonohomegenous due to the wind forcing). To satisfy the closed boundary condition at $x=L$ as well as the matching conditions between ramp-up zone and the rest of the inner region, we introduce two sets of $M+1$ collocation points at $x=L$ and $x=L_{\text {ramp }}$, respectively. See Appendix 3.C (and also Chen et al, 2015).

- A two-dimensional vertical (2DV) analytical solution can be found in the case without rotation $(f=0)$, with along-basin wind $\left(\theta=0^{\circ}\right)$, for the uniform depth or abrupt step topographies. This is detailed in Appendix 3.D.

\subsection{Results and discussion}

\subsubsection{Introduction}

We consider a large-scale reference basin, with characteristics as shown in Table 3.1. To quantify the influence of wind and topography on the water levels in the basin, we define the amplification factor $A$ as the dimensionless elevation amplitude averaged over the right boundary at $x=L$, i.e.

$$
A=\frac{\overline{|N|}}{N_{\text {ref }}}
$$

with average amplitude $\overline{|N|}$ and reference amplitude $N_{\text {ref }}$ given by

$$
\overline{|N|}=\frac{1}{B} \int_{0}^{B}\left|N_{1}(L, y)\right| \mathrm{d} y, \quad N_{\text {ref }}=\frac{\hat{T}}{g h_{\mathrm{c}} k_{\mathrm{c}}} .
$$

Here, $h_{\mathrm{c}}$ and $k_{\mathrm{c}}$ are the depth and wave number, respectively, that apply in the coastal part of the inner region (see Table 3.1 and Eq.(3.45) in Appendix 3.D). For the uniform depth case, we take $h_{\mathrm{c}}=h_{0}$ and $k_{\mathrm{c}}=k_{0}$. Physically, the 
reference amplitude $N_{\text {ref }}$ follows from balancing the pressure gradient of a shallow water wave $g k_{\mathrm{c}} N_{\text {ref }}$ with the acceleration associated with the wind stress $\left(\hat{T} / h_{\mathrm{c}}\right)$.

In presenting the model results, the frequency response is plotted as a function of a dimensionless forcing frequency $\omega / \omega_{\text {ref }}$, where the reference frequency is the frequency for which the shallow water wavelength of a basin with uniform depth equals the length of the basin:

$$
\omega_{\mathrm{ref}}=\sqrt{g h_{0}} \frac{2 \pi}{L}
$$

This remainder of this section is organised as follows. First, in $\S 3.4 .2$ we show the influence of wind direction on the frequency response for the uniform depth. Then, in $§ 3.4 .3$ and $\S 3.4 .4$ we investigate the influence of step height for the abrupt step case as well as the influence of the slope length in the smoothened step case. Then, $\S 3.4 .5$ and $\S 3.4 .6$ contain the results for the linear along-basin slope and the parabolic cross-basin profile.

\subsubsection{Uniform depth; influence of wind direction}

The influence of wind direction on frequency response is shown in Figure 3.2. The colour plots show the amplification factor $A$ for non-rotating basins of uniform depth (topography type 1), as a function of the dimensionless frequency and wind angle. Note that wind angle is important as the open boundary introduces an essential difference between along-basin dynamics and cross-basin dynamics (contrasting the closed basin study by Chen et al, 2015). The response to along-basin wind has been obtained with the analytical 2DV solution; the response to cross-basin wind with the collocation solution (as outlined at the end of §3.3.3). Then, Eq.(3.9) has been applied to obtain the response to wind with an arbitrary angle.

The response to along-basin wind is independent of basin width. More specifically, the amplification factor $A$ is zero at $\omega / \omega_{\text {ref }} \approx 0,1, \cdots$ and local maxima in between. This amplification pattern can be explained by systematically discussing the contributions to the solution in a one-dimensional case (ignoring the ramp-up region by taking the limit $L_{\text {ramp }} \downarrow 0$ and hence $\varphi_{\text {ramp }} \downarrow 0$ in Appendix 3.D).

- The wind-driven flow at the closed boundary needs to be compensated by adding a contribution $\propto \cos (k[x-L])$ to the flow field, which together produce zero velocity at $x=L$ (and also zero elevation).

- If $k L=0,2 \pi, \cdots$, i.e., if $\omega / \omega_{\text {ref }}=p$, this superposition also has zero velocity and elevation at the open boundary $(x=0)$, by which - in this case - no outgoing wave occurs.

- If $k L \neq 0,2 \pi, \cdots$, this superposition has nonzero velocity and elevation at the open boundary $(x=0)$, which are 90 degrees out of phase and hence cannot be matched with an outgoing propagating wave at that interface. 
(a) $B / L=1 / 4$
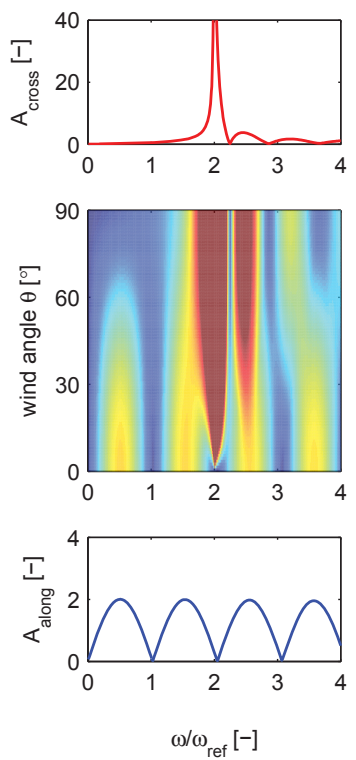

(b) $B / L=1 / 2$
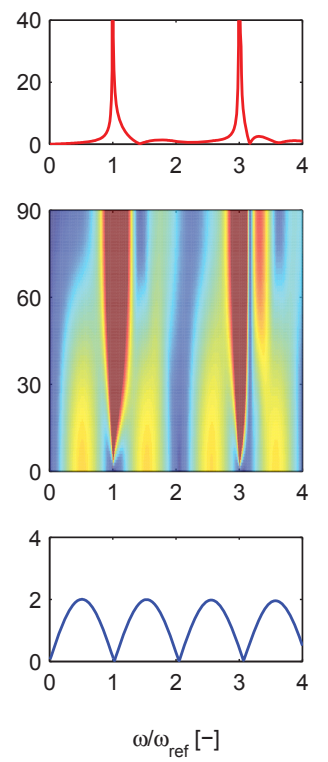

(c) $B / L=1$
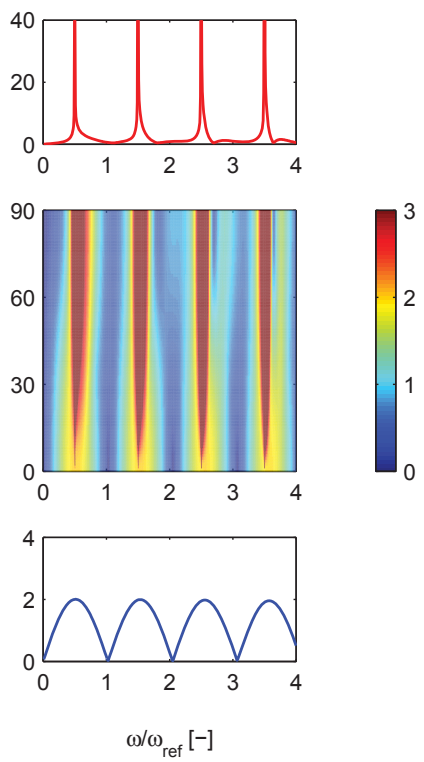

Figure 3.2: Influence of wind angle on frequency response for non-rotating basin of uniform depth, for three width-to-length ratios: (a) $B / L=1 / 4$, (b) $B / L=1 / 2$, (c) $B / L=1$. The colour plots show the amplification factor $A$ as a function of the dimensionless frequency $\omega / \omega_{\text {ref }}$ and wind angle $\theta$. The top and bottom panels show the frequency responses upon which the colour plots are based according to Eq.(3.9): $A_{\text {cross }}$ for cross-basin wind $\left(\theta=90^{\circ}\right.$, red) and $A_{\text {along }}$ for along-basin wind $\left(\theta=0^{\circ}\right.$, blue). As indicated by the different vertical scales, the peaks of the response to crossbasin wind are much higher than those for along-basin wind. Parameter values as in Table 2.1. 
- Hence, a second contribution $\propto \sin (k[x-L])$ must be added to the flow field in order to construct a solution at $x=0$ that can be matched by an outgoing propagating wave. Note that this leaves the velocity at $x=L$ unaffected.

- This second contribution to the velocity adds a nonzero contribution to the elevation at $x=L$, which is maximum for $k L=0,2 \pi, \cdots$, i.e., if $\omega / \omega_{\text {ref }}=p+\frac{1}{2}$, thus explaining the amplification pattern in the bottom panels of Fig.3.2.

The slight deviation in the bottom panel of Fig.3.2 from the exact integer values of $\omega / \omega_{\text {ref }}$ as explained above is due to the ramp-up of the wind forcing from $x=0$ to $x=L_{\text {ramp. }}$. If the basin were closed at $x=0$ (instead of connected to an outer region where waves radiate away), the local maxima would be true resonance peaks with much higher values (Chen et al, 2015).

Since both boundaries in the lateral direction at $y=0, B$ are closed, the response to cross-basin wind is much stronger than that to along-basin wind. Furthermore, the pattern strongly depends on basin width. To illustrate this, we choose three different width-to-length ratios, ranging from elongated $(B / L=$ $1 / 4)$ to square $(B / L=1)$; see Figs.3.2a-c. The cross-basin response is further influenced by the connection to the outer region, leading to small wiggles to the right of the peaks. This is different from a purely closed basin case (Chen et al, 2015). Finally, the gradual ramp-up of the wind field is crucial in the case of cross-basin wind. An abrupt transition at $x=0$ from no wind $(x<0)$ to full wind $(x>0)$ would produce unrealistically strong amplification around $(x, y)=(0,0)$ and $(0, B)$.

The colour plots in the middle panels of Fig.3.2 show how the amplification factor $A$, according to Eq.(3.9), depends on the wind angle $\theta$. The response to cross-basin wind, due to its higher peaks, appears to dominate this pattern already for relatively small wind angles.

Figure 3.3 shows the influence of the Coriolis effect on the frequency response to cross-basin wind (top panels) and along-basin wind (bottom panels). Each plot contains a curve without rotation $\left(f=0\right.$ for $\left.\vartheta=0^{\circ}\right)$ and with rotation $\left(f \neq 0\right.$, as obtained for $\left.\vartheta=50^{\circ} \mathrm{N}\right)$. Because of the Coriolis-induced interaction between along-basin and cross-basin dynamics, the cross-basin peaks emerge also in the along-basin frequency responses. Further, peaks arise exactly in between the already existing peaks, e.g. at $\omega / \omega_{\text {ref }}=2$ in Fig.3.3b, which corresponds to a cross-basin eigenmode. This mode, suppressed by symmetry for $f=0$, now emerges as the symmetry is broken by the Coriolis effect. Finally, both responses display a peak close to the inertial frequency $(f=\omega)$, which is invisible in the upper plots as the magnitude of the peaks is relatively small.

\subsubsection{Abrupt topographic step}

We will now investigate the influence of an abrupt topographic step, i.e. type 2 of the topographies introduced in $\S 3.2 .1$ and Fig.3.1. Figure 3.4a shows the 

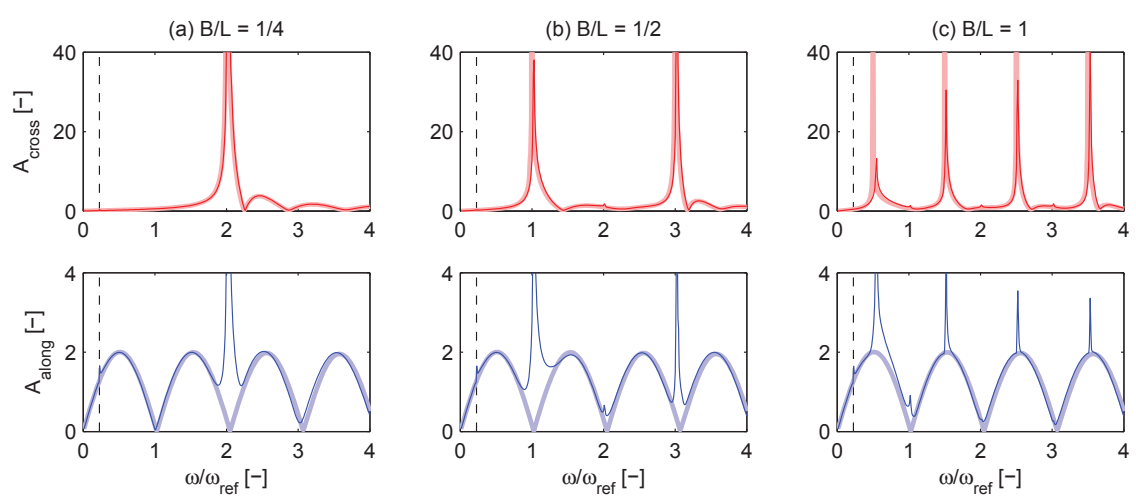

Figure 3.3: Influence of the Coriolis effect on frequency response to cross-basin wind (top panels) and along-basin wind (bottom panels), for three width-to-length ratios: (a) $B / L=1 / 4$, (b) $B / L=1 / 2$, (c) $B / L=1$. The thick background curves represent the responses of the non-rotating basin $\left(\vartheta=0^{\circ}\right)$, the sharp lines represent the rotating basin $\left(\vartheta=50^{\circ} \mathrm{N}\right)$. The vertical dashed line indicates the inertial frequency $\omega=f$. Parameter values as in Table 2.1.

frequency response for a non-rotating reference basin subject to along-basin wind. We present the following examples:

I Uniform depth (pink curve), with a depth of $h_{0}=100 \mathrm{~m}$, which is in fact identical to the blue curve in the bottom panels of Fig.3.2.

II Topographic step (black), i.e. the abrupt step case with a shallow coastal part of depth $h_{\mathrm{c}}=15 \mathrm{~m}$. Compared to the curve for uniform depth changes, we now see that the maxima become distorted and they furthermore shift to lower frequencies.

II' Same as example II, but now including the Coriolis effect (taking a latitude $\left.\vartheta=50^{\circ} \mathrm{N}\right)$. This case will be discussed further below.

The influence of step height is then investigated by varying the coastal depth $h_{\mathrm{c}}$ from $10 \mathrm{~m}$ to $1000 \mathrm{~m}$, while keeping $h_{0}=100 \mathrm{~m}$. This leads to a depth ratio $h_{\mathrm{c}} / h_{0}$ ranging from 0.1 to 10 . The resulting frequency response for this range of $h_{\mathrm{c}} / h_{0}$-values is shown in Fig $3.4 \mathrm{~b}$, where the red and blue colours indicate high and low amplification, respectively. The $h_{\mathrm{c}} / h_{0}$-values of examples I and II are indicated by the pink and black dashed horizontal lines, respectively.

The thick white lines follow local maxima of the amplification factor $|A|$, for increasing values of $h_{\mathrm{c}} / h_{0}$. In the bottom part of the figure, i.e. for $h_{c} / h_{0} \ll 1$, the peaks align with the thin solid white curves, for which the length $L_{\mathrm{c}}$ of the coastal part is an odd multiple of the quarter wave length on the coastal step. These lines are characterised by

$$
L_{\mathrm{c}}=\frac{1}{4}(2 p+1) \lambda_{\mathrm{c}}, \quad \lambda_{\mathrm{c}}=\frac{2 \pi}{k_{\mathrm{c}}}
$$



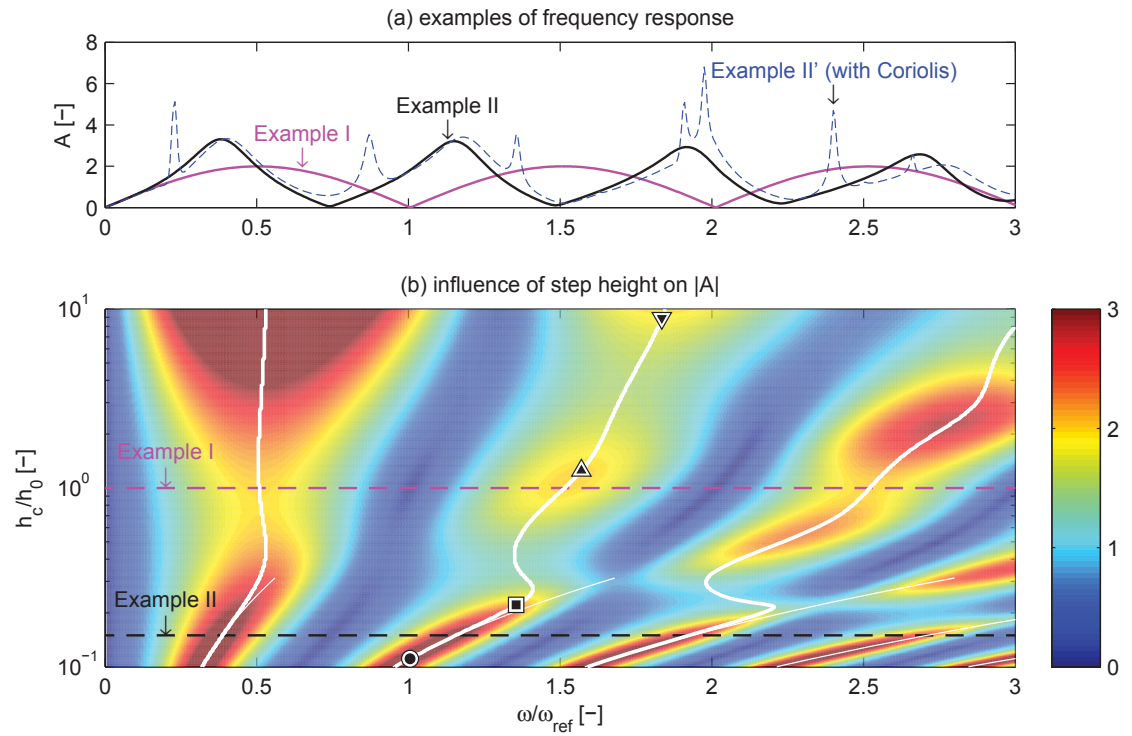

Figure 3.4: Frequency response for a non-rotating basin with uniform depth and abrupt step. (a) Amplification factor $A$ as a function of dimensionless frequency $\omega / \omega_{\text {ref }}$ for two examples: (I) uniform depth (pink curve), (II) topographic step (black). The dashed blue curve corresponds to Example II' (same as example II but now including Coriolis effect). (b) Dependency of $A$ on depth ratio $h_{\mathrm{c}} / h_{0}$. Thick white lines follow the local maxima; four cases denoted by circle, square and triangles are further illustrated in Fig.3.5. This figure has been obtained by varying $\omega$ and the depth of coastal part $h_{\mathrm{c}}$. Parameter values as in Table 2.1, with $\vartheta=0^{\circ}$. 

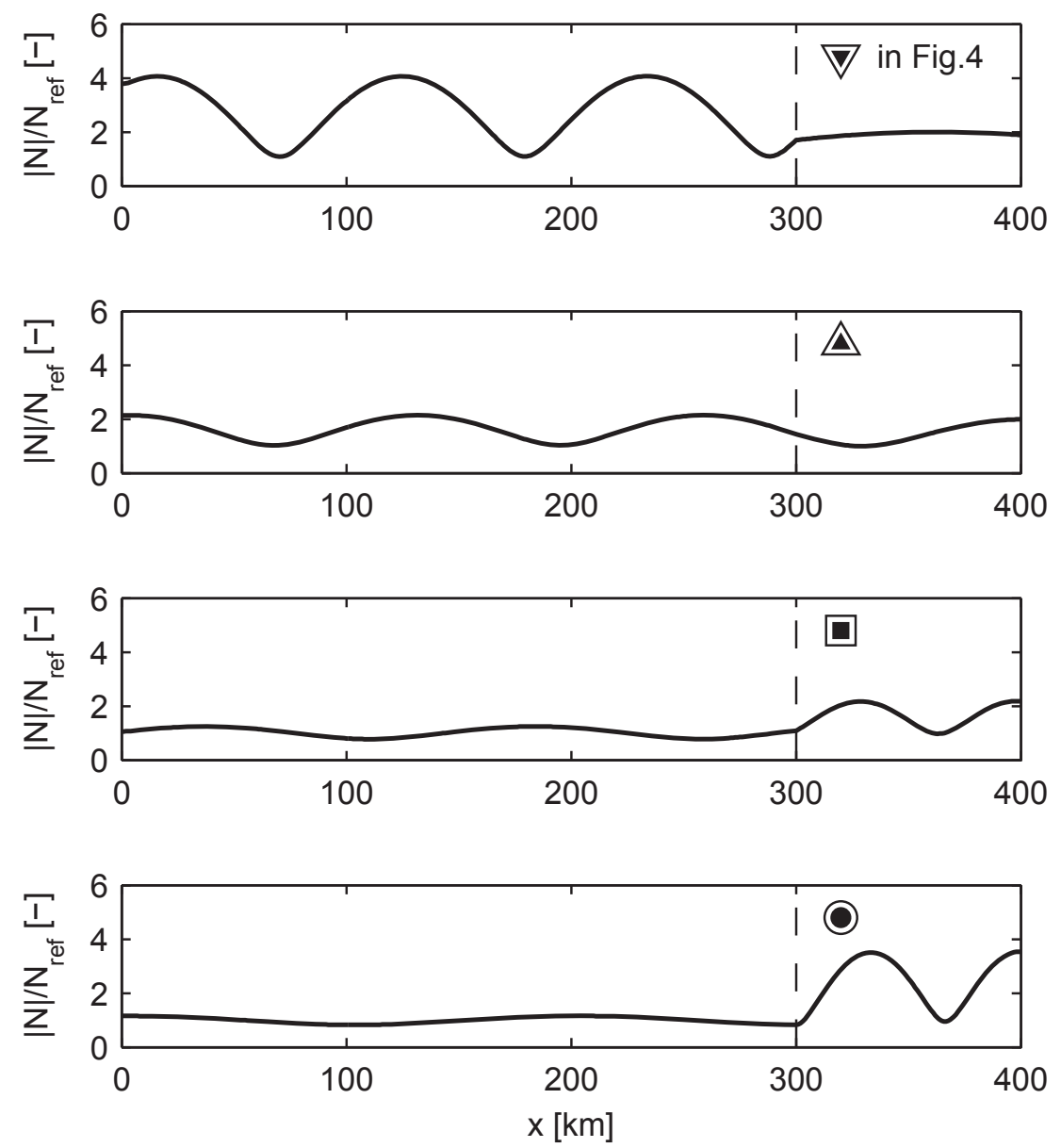

Figure 3.5: Spatial structure of the absolute value of the dimensionless elevation amplitude $|N(x)| / N_{\text {ref }}$ for the four cases denoted by a circle, a square, an upward pointing triangle and a downward triangle in Fig.3.4. The vertical dashed line indicates the position of the topographic step. For further explanation, see text. The ramp-up region has a length of $L_{\mathrm{ramp}}=10 \mathrm{~km}$. 
for $p=0,1, \cdots$. Let us consider the case $p=1$, which corresponds to the three-quarter wavelength resonance of the (shallow) coastal part. For increasing

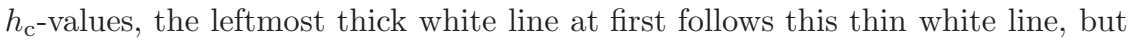
then shifts to a lower frequency. This is accompanied by an elevation node moving out of the coastal part, because the wavelength in the coastal part becomes larger as $h_{\mathrm{c}}$ increases. This indicates the gradual transition towards a spatially uniform elevation pattern in the coastal part ('pumping mode'). This gradual transition is illustrated by the four plots in Fig.3.5, showing the spatial structures of the elevation amplitudes for each of the four cases in Fig.3.4. For the other thick white curves, obtained for $p=0$ and $p=2,3, \cdots$ in Eq.(3.23), this gradual process is similar but involves a different number of elevation nodes that subsequently move out of the coastal part. Note that, due to friction, the elevation nodes referred to here have a small nonzero amplitude rather than a zero value.

To illustrate the influence of the Coriolis effect on these results, Fig.3.4 also contains the frequency response for example II', which is the same as example II but now in a rotating basin (latitude set to $\vartheta=50^{\circ} \mathrm{N}$ ). The Coriolis effect introduces new peaks, associated with cross-basin resonances in the (shallow) coastal part, with different along-basin and cross-basin structures. It should be noted that, to calculate this result in our FEM-model, the abrupt step had to be smoothened. We chose a slope length of $L_{\text {slope }}=5 \mathrm{~km}$. The effect of smoothening topographic steps will be investigated in more detail in the next subsection.

\subsubsection{Influence of slope length (smoothened step)}

The topographic steps studied in $\S 3.4 .3$ have a discontinuity in depth. In reality, however, such transitions are more gradual. To investigate this, we now consider a more smooth transition from $h_{0}$ to $h_{\mathrm{c}}$ over a length $L_{\text {slope }}$ and study this with the FEM-model, restricting to a non-rotating basin $(f=0)$. The topography around the smoothened step, as depicted in Fig.3.1 (type 3), is written as

$$
h_{1}(x)=h_{0}+\left(h_{\mathrm{c}}-h_{0}\right) F\left(x-x_{1}\right),
$$

with dimensionless transition function $F(\xi)$ chosen to be of sinusoidal shape:

$$
F(\xi)=\frac{1}{2}+\frac{1}{2} \sin \left(\frac{\pi \xi}{L_{\text {slope }}}\right), \quad-\frac{L_{\text {slope }}}{2} \leq \xi \leq \frac{L_{\text {slope }}}{2} .
$$

Figure 3.6a shows the influence of varying the slope length $L_{\text {slope }}$ on the frequency response, as obtained with our FEM-model. We again consider the reference depths $h_{0}=100 \mathrm{~m}$ and $h_{\mathrm{c}}=15 \mathrm{~m}$ as given in Table 2.1 and $L_{\text {slope }^{-}}$ values ranging from 5 to $40 \mathrm{~km}$. Importantly, the average depth of the inner region is the same for all $L_{\text {slope-values. The result shows that, for increasing }}$ values of $L_{\text {slope }}$, the frequency of the peaks shift to slightly higher values. This shift can be explained as follows. The more smooth the step (while maintaining the average depth), the larger the basin-average of $\sqrt{g h}$, which is a proxy for 


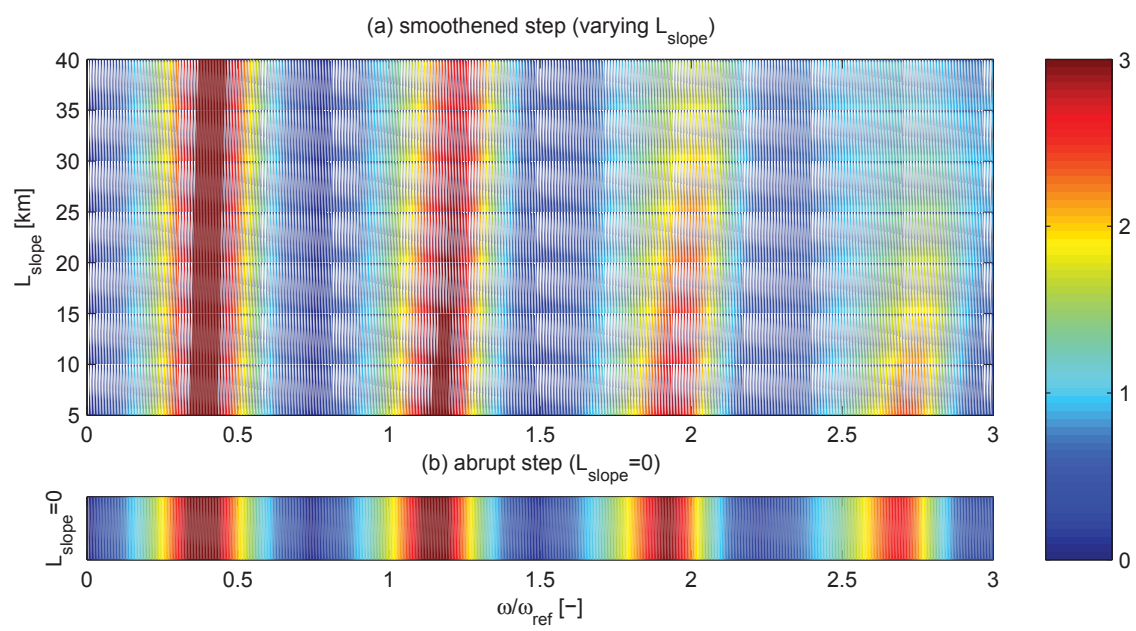

Figure 3.6: Influence of slope length on frequency response. (a) Amplification factor $A$ as a function of dimensionless frequency $\omega / \omega_{\text {ref }}$ and slope length $L_{\text {slope }}$, as obtained with our FEM-model. (b) Frequency response for an abrupt topographic step $\left(L_{\text {slope }}=\right.$ 0 ), as obtained with the 2DV-solution method, as indicated by the black dashed curve in Fig.3.4. Parameter values: $h_{\mathrm{c}}=15 \mathrm{~m}, h_{0}=100 \mathrm{~m}$.

the wave speed in the basin. Such an increase in effective wave speed reduces the travel time of waves around the basin, which implies that resonance occurs at higher frequencies.

Finally, in the limit of very small slope lengths, the frequency response as obtained with our FEM-model converges to the results of the abrupt topographic step studied in $\S 3.4 .3$ (see Fig.3.6b). On the other hand, for very large slope lengths, the step becomes so gradual that it resembles a linear profile, to be studied next.

\subsubsection{Linear profile}

The influence of a bed with a linear along-basin slope in the inner region (type 4) on the frequency response of a coastal basin, restricting to along-basin wind, is investigated by fixing the depth in the outer and ramp-up region to its reference value $h_{0}=100 \mathrm{~m}$. The depth $h_{\mathrm{c}}$, that is attained at the coast $(x=L)$, is then varied from $100 \mathrm{~m}$ to $10 \mathrm{~m}$. Maintaining uniformity in the cross-basin direction, the bed slope $S$ in the inner region is thus given by

$$
S=\frac{h_{0}-h_{\mathrm{c}}}{L-L_{\mathrm{ramp}}} .
$$

Figure 3.7 shows the frequency responses, both with and without Coriolis effect, obtained with the FEM-model. The responses for zero slope $(S=0)$ are identical to the curves in the bottom plot of Fig.3.3a. With the Coriolis effect, 
(a) without Coriolis effect $\left(\vartheta=0^{\circ}\right)$

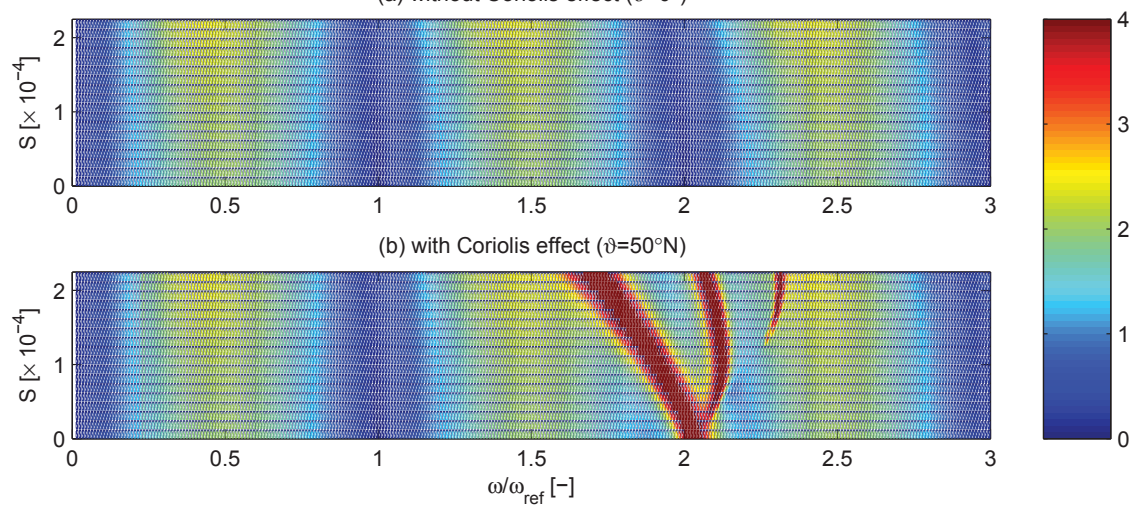

Figure 3.7: Influence of the bed slope in the inner region on the frequency response to along-basin wind. Amplification as a function of dimensionless frequency $\omega / \omega_{\text {ref }}$ and bed slope $S=\left(h_{0}-h_{\mathrm{c}}\right) /\left(L-L_{\mathrm{ramp}}\right)$ in two cases: (a) without the Coriolis effect, (b) with the Coriolis effect.

the frequency response includes peaks associated with cross-basin resonances, the frequency of which becomes smaller as $S$ increases. Furthermore, as the slope is increased, more peaks appear that are also associated with cross-basin resonances, but differ in their along-basin structures. Away from these resonance peaks and regardless of the Coriolis effect, increasing the bed slope leads to higher amplification, the maxima of which are shifted to slightly lower frequencies.

\subsubsection{Parabolic cross-basin profile}

Finally, we investigate the influence of a parabolic cross-basin depth profile (type 5) on the frequency response, restricting to along-basin wind. The shape, depicted in Fig.3.8, is given by

$$
h(x, y)=h_{0}+\Delta h F\left(x-x_{2}\right)\left[1-12\left(\frac{y}{B}-\frac{1}{2}\right)^{2}\right],
$$

with parameter $\Delta h$, dimensionless transition function $F(\xi)$ as already introduced in Eq.(3.25), to be centered around $x=x_{2}$ with $x_{2}=L_{\text {ramp }}+\frac{1}{2} L_{\text {slope }}$. The profile is chosen such that the width-averaged depth is equal to $h_{0}$. The depth at the centerline is given by $h_{0}+\Delta h$, the depth at the banks $y=0, B$ is given by $h_{0}-2 \Delta h$. Importantly, the dimensionless transition function $F(\xi)$ ensures a smooth transition from the spatially uniform depth $h_{0}$ in the outer region and ramp-up region to our parabolic cross-basin profile in the inner region.

Using the reference value $h_{0}=100 \mathrm{~m}$, we vary $\Delta h$ from 0 to $40 \mathrm{~m}$, giving a ratio $\Delta h / h_{0}$ between 0 and 0.4 . Figure 3.9 shows the frequency responses, both with and without Coriolis effect, obtained with the FEM-model. The responses 
(a) along-basin side view

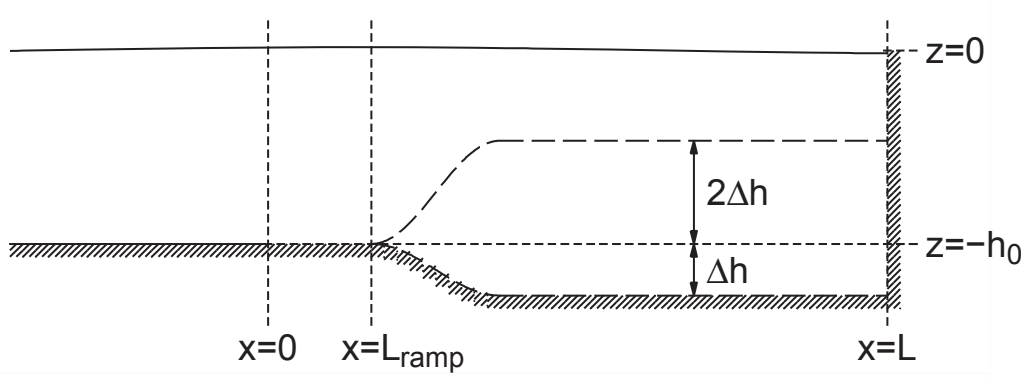

(b) cross-basin side view

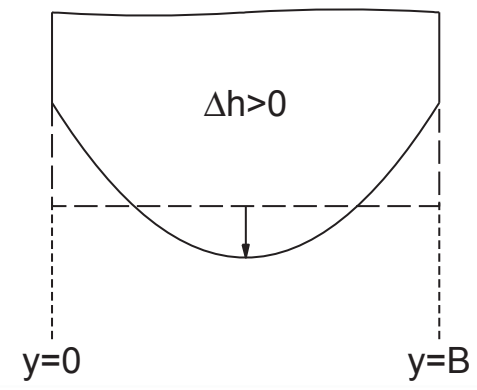

Figure 3.8: Definition sketch of parabolic profile. (a) along-basin side view showing gradual transition from uniform depth $h_{0}$ in outer and ramp-up region to parabolic cross-basin profile in inner region. The dashed lines at $z=-h_{0}-\Delta h$ and $z=$ $-h_{0}+2 \Delta h$ indicate the water depth at the centerline and the banks, respectively. (b) Cross-basin side view in the inner region, showing the parabolic profile according to Eq.(3.27). 


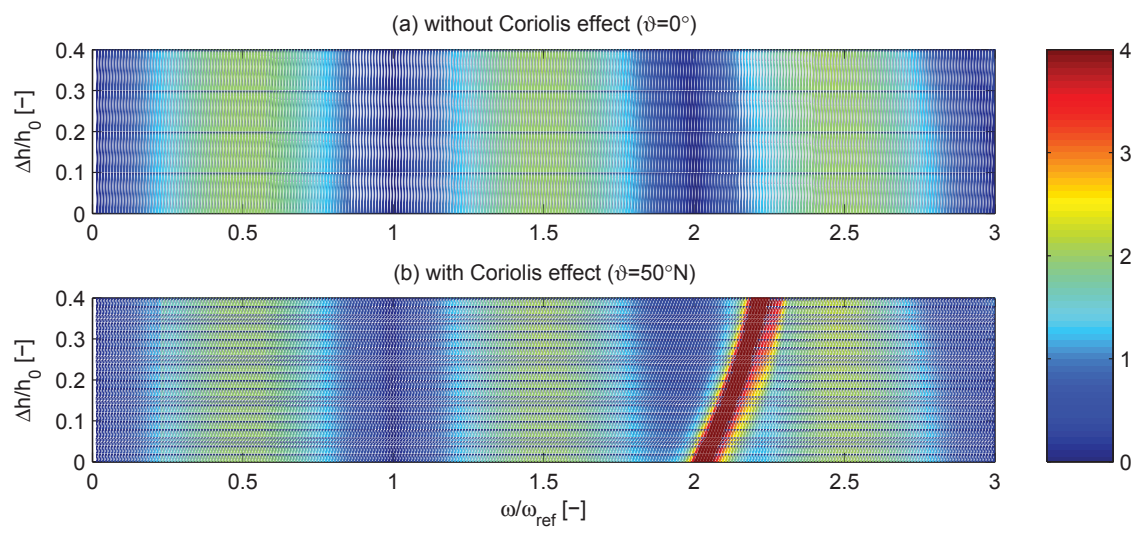

Figure 3.9: Same as Fig.3.7, but now the influence of parabolic cross-basin profile on the frequency response as a function of $\Delta h / h_{0}$.

for $\Delta h=0$, corresponding to a uniform depth, are in fact identical to the curves in the bottom plot of Fig.3.3a.

Without Coriolis effect, for increasing values of $|\Delta h|$, the local maxima of the amplification shift to lower frequencies. This can be explained by the effective wave speed that is based on the cross-basin average of $\sqrt{g h}$, as mentioned in $\S 3.4 .4$. Indeed, the result of increasing $|\Delta h|$ is qualitatively similar to that of reducing the slope length $L_{\text {slope }}$ for the smoothened step case. The results with the Coriolis effect show a similar 'background' amplification pattern as obtained for $\vartheta=0^{\circ}$. In addition to that, the Coriolis effect introduces peaks associated with cross-basin resonances with peak frequencies that shift to higher values for increasing $\Delta h$.

\subsection{Conclusions}

We have developed an idealised process-based model to analyse the influence of specific topographic elements on the frequency response of semi-enclosed coastal basins subject to time-periodic wind forcing. Coastal basins are represented by a large-scale semi-enclosed rectangular inner region where the wind forcing takes place and where a variety of topographic elements have been included. It is connected to an outer region, without wind forcing and stretching to infinity, which allows waves to freely propagate outward. The model solves the threedimensional linearised shallow water equations on the $f$ plane, forced by a wind field that ramps up to a spatially uniform pattern in the core of the inner region. The wind field has an arbitrary angle with respect to the along-basin direction. The model solves a two-dimensional problem for the surface elevation amplitudes by applying the Finite Element Method (FEM), extended to account for the outward propagating waves in the outer region. In particular cases, alternative (semi-)analytical solution techniques are used. 
By restricting to linear dynamics, we have neglected nonlinear effects. This facilitates our understanding in two ways: $(i)$ the response to any wind event is contained in the frequency response, $(i i)$ the frequency response for arbitrary wind angle is in fact a linear combination of the frequency responses to alongbasin and cross-basin wind. The responses to along-basin and cross-basin wind are essentially different because of the differences in the along-basin and crossbasin dynamics caused by the open boundary.

To analyse our model, we focused on the elevation amplitude averaged over the basin's landward end, as a function of the dimensionless frequency $\omega / \omega_{\text {ref }}$ with reference frequency $\omega_{\text {ref }}=\sqrt{g h_{0}} 2 \pi / L$ for which the shallow water wavelength (of the basin with uniform depth) equals the length of the basin. For cases not including the Coriolis effect, we conclude the following.

1. In the reference case of a uniform depth, the response to along-basin wind is a pattern showing zero amplification at $\omega / \omega_{\text {ref }} \approx 0,1,2, \cdots$ and maximum amplification in between. Due to the waves allowed to propagate away into the outer sea, these maxima are found to be much weaker than the peaks in the frequency response to cross-basin wind.

2. For a topographic step with a shallow coastal part, we observe the resonance frequencies associated with (odd multiples of) the quarter wavelength, which are known to increase when increasing the coastal depth. Conversely, when sufficiently deep, the coastal part displays a spatially uniform elevation pattern ('pumping mode', see top panel of Figure 3.5).

3. Smoothening the steps shows that increasing the slope length shifts the maximum to slightly higher frequencies.

4. The response to a linear along-basin bed profile in the inner region (alongbasin wind only) shows higher response for increasing slopes (while fixing the depth in the outer region), because of the reduced depth in the coastal part.

5. The response to a parabolic cross-basin profile (along-basin wind only) shows that varying the central depth, while keeping the average depth the same, only weakly modifies the frequency response.

The Coriolis effect causes the (strong) resonance peaks associated with crossbasin modes (which without rotation only appear in the response to cross-basin wind) to emerge also in the response to along-basin wind. It also introduces peaks at the inertial frequency.

This study is a first step towards understanding the responses of natural basins to wind forcing. In particular, our modelling approach also applies to basins with a topography that is more complicated than the schematised representation used in this study. Furthermore, extending the model with respect to atmospheric forcing (e.g. representing the moving low-pressure system of a hurricane) and geometry (coastlines) is a subject of ongoing research. 
Acknowledgement: This work is partly funded by the Chinese Scholarship Council and partly by the research programme 'Impact of climate change and human intervention on hydrodynamics and environmental conditions in the Ems-Dollart estuary: an integrated data-modelling approach'. The latter project is financed by the Bundesministerium fur Bildung und Forschung (BMBF) and by the Netherlands Organization for Scientific Research (NWO), as part of the international Wadden Sea programme (GEORISK project). 


\section{Appendix}

\section{A Ramp-up function}

The ramp-up function $\mu(x)$ of the wind describes the transition from zero wind in the outer region to a spatially uniform wind field, in a region of length $L_{\text {ramp }}$ (see Fig.3.1c). It is given by

$$
\mu(x)= \begin{cases}0 & \text { if } x<0 \\ \frac{\sin k x}{\sin k L_{\mathrm{ramp}}} & \text { if } 0 \leq x \leq L_{\mathrm{ramp}} \\ 1 & \text { if } x>L_{\mathrm{ramp}}\end{cases}
$$

Here, $k$ is the wave number as given in Eq.(3.45) in Appendix 3.D. The motivation for this particular shape of the ramp-up function, which through $k$ depends on the problem parameters, lies in the solution method. In fact, only this choice allows us to obtain analytical solutions (Appendix 3.D) and collocation solutions (Appendix 3.C) for the cases indicated in $\S 3.3 .3$. To have a monotonically increasing ramp-up function, i.e. without any oscillations, we choose $L_{\text {ramp }}$ such that $k L_{\text {ramp }}<\pi / 2$.

\section{B Details of the derivation}

\section{B.1 Vertical profiles from horizontal momentum equations}

Here we present the details of the vertical structure of the flow. First we define rotating flow components according to $q^{ \pm}=u \pm i v$ with complex amplitudes $Q^{ \pm}$, such that $U=\left(Q^{+}+Q^{-}\right) / 2$ and $V=\left(Q^{+}-Q^{-}\right) /(2 i)$. The rotating flow solution contains three contributions, proportional to the surface gradient the wind stress and the pressure gradient, respectively:

$$
Q^{ \pm}(z)=Q_{\eta}^{ \pm}(z) \mathcal{L}^{ \pm} N+Q_{\mathrm{w}}^{ \pm}(z) T^{ \pm}
$$

with complex operators $\mathcal{L}^{ \pm}=\partial / \partial x \pm \mathrm{i} \partial / \partial y$ and rotating wind forcing amplitudes $T^{ \pm}=T^{(x)} \pm \mathrm{i} T^{(y)}$ (wind stress divided by density). The vertical structures read

$$
\begin{aligned}
Q_{\eta}^{ \pm}(z) & =\frac{g\left[\cosh \lambda^{+} z-\alpha_{\mathrm{c}}^{ \pm}\right]}{\alpha_{\mathrm{c}}^{ \pm} K \lambda^{ \pm 2}}, \\
Q_{\mathrm{w}}^{ \pm}(z) & =\frac{\alpha_{\mathrm{c}}^{ \pm} \sinh \lambda^{ \pm} z+\alpha_{\mathrm{s}}^{ \pm} \cosh \lambda^{ \pm} z}{\alpha_{\mathrm{c}}^{ \pm} K \lambda^{ \pm}} .
\end{aligned}
$$

with $\lambda^{ \pm 2}=-\mathrm{i}(\omega \mp f) / K$ and $\alpha_{\mathrm{c}}^{ \pm}=\cosh \lambda^{ \pm} h+s^{-1} K \lambda^{ \pm} \sinh \lambda^{ \pm} h$ and $\alpha_{\mathrm{s}}^{ \pm}=\sinh \lambda^{ \pm} h+$ $s^{-1} K \lambda^{ \pm} \cosh \lambda^{ \pm} h$. The vertical integral is given by

$$
\left\langle Q^{ \pm}\right\rangle=\left\langle Q_{\eta}^{ \pm}\right\rangle \mathcal{L}^{ \pm} N+\left\langle Q_{\mathrm{w}}^{ \pm}\right\rangle T^{ \pm},
$$

with

$$
\begin{aligned}
\left\langle Q_{\eta}^{ \pm}\right\rangle & =\frac{g\left[\sinh \lambda^{ \pm} h-\alpha_{\mathrm{c}}^{ \pm} \lambda^{ \pm} h\right]}{\alpha_{\mathrm{c}}^{ \pm} K \lambda^{ \pm 3}}, \\
\left\langle Q_{\mathrm{w}}^{ \pm}\right\rangle & =\frac{\alpha_{\mathrm{c}}^{ \pm}\left[1-\cosh \lambda^{ \pm} h\right]+\alpha_{\mathrm{s}}^{ \pm} \sinh \lambda^{ \pm} h}{\alpha_{\mathrm{c}}^{ \pm} K \lambda^{ \pm 2}} .
\end{aligned}
$$


The two cases $\omega= \pm f$ require alternative expressions for either $Q^{+}$or $Q^{-}$. If $\omega=+f$ we must replace the $Q^{+}$-expressions in Eqs.(3.30)-(3.32); if $\omega=-f$ we must replace the $Q^{-}$-expressions. They must be replaced with

$$
\begin{aligned}
& Q_{\eta}^{ \pm}(z)=\frac{g h^{2}}{K}\left[\frac{1}{2}\left(\frac{z}{h}\right)^{2}-\frac{1}{2}-\frac{K}{s h}\right], \\
& Q_{\mathrm{w}}^{ \pm}(z)=\left[1+\left(\frac{z}{h}\right)+\frac{K}{s h}\right],
\end{aligned}
$$

and

$$
\left\langle Q_{\eta}^{ \pm}\right\rangle=-\frac{g h^{3}}{K}\left[\frac{1}{3}+\frac{K}{s h}\right], \quad\left\langle Q_{\mathrm{w}}^{ \pm}\right\rangle=\frac{h^{2}}{K}\left[\frac{1}{2}+\frac{K}{s h}\right] .
$$

\section{B.2 Elliptical problem for $N$}

Depth-integration of the continuity equation (3.3), with the aid of boundary conditions (3.4) gives, in terms of the complex amplitudes of surface elevation and the rotating velocity components.

$$
-\mathrm{i} \omega N+\frac{\partial}{\partial x} \underbrace{\left(\frac{\left\langle Q^{+}\right\rangle+\left\langle Q^{-}\right\rangle}{2}\right)}_{\langle U\rangle}+\frac{\partial}{\partial y} \underbrace{\left(\frac{\left\langle Q^{+}\right\rangle-\left\langle Q^{-}\right\rangle}{2 \mathrm{i}}\right)}_{\langle V\rangle}=0,
$$

Substitution of Eq.(3.29) gives the elliptical equation for $N$ presented in Eq.(3.12) of the main text. The corresponding coefficients are given by

$$
C^{+}=\frac{1}{2}\left[Q_{\eta}^{+}+Q_{\eta}^{-}\right], \quad C^{-}=\frac{1}{2 \mathrm{i}}\left[Q_{\eta}^{+}-Q_{\eta}^{-}\right],
$$

The boundary conditions presented in Eqs.(3.14)-(3.15) of the main text follow from depth-integration of the momentum equations (3.1)-(3.2). The coefficients in there are given by

$$
R^{+}=\frac{1}{2}\left[Q_{\mathrm{w}}^{+} T^{+}+Q_{\mathrm{w}}^{-} T^{-}\right], \quad R^{-}=\frac{1}{2 \mathrm{i}}\left[Q_{\mathrm{w}}^{+} T^{+}-Q_{\mathrm{w}}^{-} T^{-}\right]
$$

\section{B.3 Vertical flow velocity}

The vertical flow velocity amplitudes at any depth $z$ are given by

$$
W(z)=-\left\lfloor C^{\oplus}\right\rfloor\left(\frac{\partial^{2} N}{\partial x^{2}}+\frac{\partial^{2} N}{\partial y^{2}}\right)-\left\langle C^{\oplus}\right\rangle\left(\left\lfloor\frac{\partial R^{\oplus}}{\partial x}\right\rfloor+\left\lfloor\frac{\partial R^{\ominus}}{\partial y}\right\rfloor\right),
$$

where floor brackets indicate integration from bottom to $z$, i.e. $\lfloor\cdot\rfloor=\int_{-h}^{z} \cdot \mathrm{d} z$. This expression can be simplified further by using the differential equation (3.12) for $N$ to eliminate the Laplacian of $N$.

\section{C Collocation method}

This appendix describes the collocation method that we apply for the uniform depth case, for cross-basin wind and $f=0$. The modifications necessary in the abrupt step case are mentioned at the end of the analysis. For uniform depth, we distinguish the solution in the ramp-up zone $\left(N_{1}\right)$ from that in the core of the inner region $\left(N_{2}\right)$. The 
problem posed in Eqs.(3.12)-(3.17), as derived in Appendix 3.B, then reduces to a nonhomogeneous Helmholtz problem:

$$
\begin{aligned}
& \frac{\partial^{2} N_{0}}{\partial x^{2}}+\frac{\partial^{2} N_{0}}{\partial y^{2}}+k^{2} N_{0}=0, \\
& \frac{\partial^{2} N_{1}}{\partial x^{2}}+\frac{\partial^{2} N_{1}}{\partial y^{2}}+k^{2} N_{1}=-\left[\frac{\partial\left\langle R_{1}^{+}\right\rangle}{\partial x}+\frac{\partial\left\langle R_{1}^{-}\right\rangle}{\partial y}\right], \\
& \frac{\partial^{2} N_{2}}{\partial x^{2}}+\frac{\partial^{2} N_{2}}{\partial y^{2}}+k^{2} N_{2}=0 .
\end{aligned}
$$

Only in the ramp-up zone there is a divergence of the wind forcing, explaining the nonzero right-hand side of Eq.(3.43). Furthermore, $k$ is a wave number satisfying

$$
k^{2}=\frac{-\mathrm{i} \omega}{\left\langle C^{+} s\right\rangle},
$$

with the depth-integrated coefficient $\left\langle C^{+}\right\rangle=\left\langle C_{0}^{+}\right\rangle=\left\langle C_{1}^{+}\right\rangle=\left\langle C_{2}^{+}\right\rangle$(uniform depth) as specified in Eq.(3.39). The boundary and matching conditions remain as in Eqs.(3.14)(3.17).

Analogous to our extended FEM-model in $§ 3.3 .3$, the solution in the outer region is written as a superposition of outgoing wave modes, i.e.

$$
N_{0}(x, y)=\sum_{m=0}^{M} c_{0, m}^{\ominus} N_{m}^{\ominus}(y) \exp \left(\mathrm{i} k_{m}^{\ominus} x\right),
$$

with coefficients $c_{0, m}^{\ominus}$. The cross-basin structures $N_{m}^{\ominus}(y)$ and wave numbers $k_{m}^{\ominus}$ are specified in appendix 3.E. The solutions in the ramp-up region and the inner region are written as a superposition of two families of wave modes plus a particular solution:

$$
\begin{gathered}
N_{j}(x, y)=N_{j, \operatorname{part}}(x, y)+\sum_{m=0}^{M} c_{j, m}^{\oplus} N_{m}^{\oplus}(y) \exp \left(\mathrm{i} k_{m}^{\oplus} x\right) \\
+\sum_{m=0}^{M} c_{1, m}^{\ominus} N_{m}^{\ominus}(y) \exp \left(\mathrm{i} k_{m}^{\ominus} x\right)
\end{gathered}
$$

The particular solutions $N_{j \text {,part }}(x, y)$ for $j=1,2$ are introduced to homogenise the cross-basin boundary conditions. For the ramp-up zone and the core of the inner region, they are given by

$$
\begin{aligned}
& N_{1, \text { part }}(x, y)=-\left(y-\frac{B}{2}\right) \frac{\left\langle Q_{\mathrm{w}}^{+}\right\rangle+\left\langle Q_{\mathrm{w}}^{-}\right\rangle}{2\left\langle C^{+}\right\rangle} \frac{\sin k x}{\sin k L_{\mathrm{ramp}}}, \\
& N_{2, \text { part }}(x, y)=-\frac{1}{k}\left(\sin k y-\frac{1-\cos k B}{\sin k B} \cos k y\right) \frac{\left\langle Q_{w}^{+}\right\rangle}{\left\langle C^{+}\right\rangle} .
\end{aligned}
$$

The solution in Eqs.(3.46)-(3.47) is thus contained in the five families of in total $5(M+1)$ coefficients $c_{0, m}^{\ominus}, c_{1, m}^{\oplus}, c_{1, m}^{\oplus}, c_{2, m}^{\oplus}$ and $c_{2, m}^{\oplus}$ (for $\left.m=0,1, \cdots, M\right)$. Their values follow from applying a collocation technique. To this end, we require the matching of surface elevation and normal transport to be satisfied at two sets of $M+1$ collocation points at $x=0$ and $x=L_{\mathrm{ramp}}$, and the closed boundary condition at another set of collocation points $x=L$. 
The analysis for the abrupt step case requires dividing the inner region into three parts: the ramp-up zone denoted with subscript $j=1$, an offshore part of depth $h_{0}(j=2)$ and a coastal part of depth $h_{\mathrm{c}}(j=3)$. The above analysis can then be repeated, distinguishing a different wave number in the coastal part and imposing similar matching conditions also at $x=x_{\text {step }}$. For brevity, the analysis is not presented here.

\section{D 2DV-solution}

This appendix contains the analytical 2DV-solution without rotation $(f=0)$, with along-basin wind $(\theta=0)$, for the uniform depth. The modifications necessary in the abrupt step case are mentioned at the end of the analysis. This solution is termed '2DV' because there is neither flow in the cross-basin direction, nor dependency on the cross-basin coordinate $y$. Hence, the flow amplitudes depend on $x$ and $z$ only, and the elevation amplitude $N(x)$ depends on the along-basin coordinate only. The problem posed in Eqs.(3.42)-(3.44), as presented in Appendix 3.C for the uniform depth case, now reduces to a one-dimensional Helmholtz problem. We thus write

$$
\begin{aligned}
& \frac{\partial^{2} N_{0}}{\partial x^{2}}+k^{2} N_{0}=0, \\
& \frac{\partial^{2} N_{1}}{\partial x^{2}}+k^{2} N_{1}=-\frac{\partial\left\langle R_{1}^{\oplus}\right\rangle}{\partial x}, \\
& \frac{\partial^{2} N_{2}}{\partial x^{2}}+k^{2} N_{2}=0 .
\end{aligned}
$$

Only in the ramp-up region there is a divergence of the wind forcing, explaining the nonzero right-hand side of Eq.(3.51). The wave number $k$ is still as given by Eq.(3.45) in Appendix 3.C, and the boundary and matching conditions in Eqs.(3.14)-(3.17) become

at $x=L$ and

$$
\left\langle C_{2}^{\oplus}\right\rangle \frac{\mathrm{d} N_{2}}{\mathrm{~d} x}=-\left\langle R_{2}^{\oplus}\right\rangle,
$$

$$
N_{j}=N_{j+1}, \quad \frac{\mathrm{d} N_{j}}{\mathrm{~d} x}=\frac{\mathrm{d} N_{j+1}}{\mathrm{~d} x},
$$

to be satisfied at $x=0$ for $j=0$ and at $x=L_{\mathrm{ramp}}$ for $j=1$. The solution is given by

$$
\begin{aligned}
& N_{0}=a \exp (-\mathrm{i} k x) \\
& N_{1}=a \exp (-\mathrm{i} k x)-c \frac{k x \sin k x}{\sin \varphi_{\text {ramp }}} \\
& N_{2}=b \cos (k[x-L])-2 c \sin (k[x-L]),
\end{aligned}
$$

with coefficients $a, b$ and $c$ given by

$$
\begin{aligned}
a & =\frac{\left\langle Q_{w}^{+}\right\rangle \hat{T}}{2 k\left\langle C_{1}^{\oplus}\right\rangle}\left[\frac{\varphi_{\mathrm{ramp}}\left(\tan \Delta \varphi-\cot \varphi_{\mathrm{ramp}}\right)-1}{(\tan \Delta \varphi-\mathrm{i}) \exp \left(\mathrm{i} \varphi_{\mathrm{ramp}}\right)}+2 \mathrm{i} \exp (-\mathrm{i} \varphi)\right], \\
b & =\frac{\left\langle Q_{w}^{+}\right\rangle \hat{T}}{2 k\left\langle C_{1}^{\oplus}\right\rangle}\left[-\mathrm{i} \exp (-\mathrm{i} \Delta \varphi)\left(1+\varphi_{\mathrm{ramp}} \cot \varphi_{\mathrm{ramp}}-\mathrm{i} \varphi_{\mathrm{ramp}}\right)+2 \mathrm{i}\right], \\
c & =\frac{\left\langle Q_{w}^{+}\right\rangle \hat{T}}{2 k\left\langle C_{1}^{\oplus}\right\rangle}
\end{aligned}
$$


with $\varphi=k L, \varphi_{\mathrm{ramp}}=k L_{\mathrm{ramp}}$, and $\Delta \varphi=k\left(L-L_{\mathrm{ramp}}\right)$.

The analysis for the abrupt step case requires dividing the inner region into three parts: the ramp-up zone denoted with subscript $j=1$, an offshore part of depth $h_{0}(j=2)$ and a coastal part of depth $h_{\mathrm{c}}(j=3)$. The above analysis can then be repeated, distinguishing a different wave number in the coastal part and imposing similar matching conditions also at $x=x_{\text {step }}$. For brevity, the analysis is not presented here.

\section{E Wave modes}

Our solution technique makes use of families of so-called Kelvin and Poincaré modes, which are eigenmodes in an infinite channel of uniform depth and width. We distinguish two families: those propagating (or exponentially decaying) in the positive $x$ direction (termed 'positive' modes, indicated with superscript $\oplus$ ) and those propagating (or exponentially decaying) in the negative $x$-direction (termed 'negative' modes, indicated with superscript $\ominus$ ).

More precisely, the solution in the outer region, as given by Eq.(3.19), is written as a truncated superposition of 'negative' Kelvin and Poincaré modes. Furthermore, the collocation technique described in Appendix 3.C uses eigenmodes also in the ramp-up and inner region. This involves both positive and negative modes, but only in the $f=0$-limit. by

For arbitrary $f$, the Kelvin mode, propagating in the positive $x$-direction, is given

$$
N_{0}(y)^{\oplus}=\exp \left(\frac{-y}{R_{\text {def }}}\right) \exp \left(\mathrm{i} k_{0}^{\oplus} x\right), \quad R_{\text {def }}=\sqrt{\frac{\left\langle\left(C_{j}^{+}\right)^{2}\right\rangle+\left\langle\left(C_{j}^{-}\right)^{2}\right\rangle}{\left\langle\left(C_{j}^{+}\right)^{2}\right\rangle k^{2}}} .
$$

with wave number

$$
k_{0}^{\oplus}=\sqrt{\frac{\left\langle\left(C_{j}^{-}\right)^{2}\right\rangle k^{2}}{\left\langle\left(C_{j}^{+}\right)^{2}\right\rangle+\left\langle\left(C_{j}^{-}\right)^{2}\right\rangle}} .
$$

The Kelvin mode propagating in the negative $x$-direction is obtained by a symmetry argument, which also implies $k_{0}^{\ominus}=-k_{0}^{\oplus}$. In the $f=0$ limit, for which $\left\langle C_{j}^{-}\right\rangle=0$, the Kelvin mode effectively becomes a shallow water wave with a uniform cross-basin structure.

The Poincaré modes, propagating or exponentially decaying in the positive $x$ direction, are given by

$$
N_{m}^{\oplus}(x, y)=\left[\cos \left(\frac{m \pi y}{B}\right)-\frac{\mathrm{i} k\left\langle C_{j}^{\oplus}\right\rangle B}{m \pi\left\langle C_{j}^{\ominus}\right\rangle} \sin \left(\frac{m \pi y}{B}\right)\right] \exp \left(\mathrm{i} k_{m}^{\oplus} x\right),
$$

for $m=1,2, \cdots$ and with the wave number $k_{m}^{\oplus}$ satisfying

$$
k_{m}^{\oplus 2}=k^{2}-\left(\frac{m \pi}{B}\right)^{2} .
$$

The Poincaré modes propagating in the negative $x$-direction are obtained by a symmetry argument, which also implies $k_{m}^{\ominus}=-k_{m}^{\oplus}$. In the limit $f=0$ the Poincaré modes obtain an elevation structure proportional to $\cos (m \pi y / B)$. here.

For brevity, the along-basin flow components of the above modes are not presented 


\title{
Chapter 4
}

\section{The influence of storm characteristics on storm surges in the New Orleans coastal basins}

\begin{abstract}
The New Orleans coastal basin is frequently attacked by hurricanes. However, from the variation in storms and storm surges, it is unclear how storm characteristics determine the surge levels and surge level distribution along the coast. In order to investigate the sensitivity of storm surges to storm characteristics, we have developed an idealised process-based model. This model solves the linearised threedimensional shallow water equations, including the Coriolis effect. It is forced by pressure and wind fields according to the Holland B model. The surge model is fast because it decouples the vertical calculations, which are analytical, from the horizontal calculations, which are carried out using the Finite Element Method. As model output, we consider the temporal pattern of the water levels (including the magnitude and timing of the peak values) at various locations along the coast. To gain confidence in the model, it is first applied to simulate the Katrina surge in the New Orleans coastal basin (2005). The model domain covers the northeastern part of the Gulf of Mexico, with a schematised representation of the coastlines and bathymetry. Model results qualitatively reproduce the observed surge levels and those simulated with the so-called ADCIRC model. Next, using the same model domain, we perform a sensitivity study in which we systematically vary the storm parameters around the values that represent Hurricane Katrina. The storm direction and the point of landfall are found to be the most important parameters. In particular, a storm approaching from south-east making landfall at the seaward end of the Mississippi dike produces the highest surge levels. This effect is further enhanced for storms with a larger storm size and a slower forward speed. Surge levels are found to also depend on the central pressure, but to a lesser extent than on the other parameters.
\end{abstract}

\subsection{Introduction}

Storm surges pose a major threat to low-lying coastal areas. This was illustrated in 2005, when Hurricane Katrina made landfall in New Orleans. The induced

${ }^{\star}$ This chapter is now under review. Chen, W.L., Straatsma, Roos, P.C., Schuttelaars, H.M., Van Ledden, M. and Hulscher, S.J.M.H. (2015, submitted). The influence of storm characteristics on storm surges in the New Orleans coastal basin. 
surge was up to $9 \mathrm{~m}$, which is the highest storm surge ever recorded in the USA (Bossak, 2005). Half of the city flooded, causing 1836 casualties and a total damage of 120 billion US\$. Interestingly, a smaller hurricane (Isaac in 2012, with a peak surge of $4 \mathrm{~m}$ ) at some locations caused higher water levels than Katrina (Fanelli and Wolcott, 2012). It is unclear why certain storms induce higher storm surges than others and why peak surges occur at different locations. Answering these questions requires understanding of the storm surge processes and the factors influencing storm surges. Below we will review the literature on this topic, paying particular attention to the storm characteristics.

The surge level that a given storm produces at a specific location is influenced by several factors. Based on the maximum sustained wind speed, the Saffir-Simpson scale (Simpson, 1974) classifies the severity of a hurricane into five categories and gives an indication of the expected surge levels (see Table 4.1). Although the Saffir-Simpson scale has historically proven to be an adequate categorization for hurricane damages, there are still exceptions. For example, Hurricane Katrina was classified as a category 3 storm (Blake et al, 2006), because the maximum sustained wind speed was $55.8 \mathrm{~m} \mathrm{~s}^{-1}$ when it made landfall for the third time* (see Table 4.1). However, it produced a $9 \mathrm{~m}$ surge, which is much higher than predicted by the Saffir-Simpson scale. On the other hand, Hurricane Charley in 2004, a category 4 storm at landfall caused surge levels corresponding to category 2 only (Pasche et al, 2005). Another complication is that, because the storm characteristics vary over time as the storm develops, also the Saffir-Simpson category may change over time.

Turning back to Hurricane Katrina, Irish et al (2008) suggested that the large storm size (measured as the distance between storm centre and location of maximum wind speed) may explain the extra high surge level. This is because a storm of a larger size not only affects a larger area, but the strong winds also tend to affect this area during a longer period of time. Another important factor is the forward speed of the storm. Varying a storm's forward motion may account for variations in flooded volumes (Rego and Li, 2009). Moreover, resonance may occur for certain values of the hurricane's forward speed (Bertin et al, 2012). Weisberg and Zheng (2006) identified the storm track as another important factor concerning the resulting storm surge when a hurricane moves over a coastal basin. For example, in Tampa Bay, Florida, a northerly approaching storm yields a higher surge than storms from other directions. Depending on the coastline, some storm tracks may trigger a forerunner, i.e. an early increase of water level in advance of landfalling storms (Redfield and Miller, 1957; Bunpapong et al, 1985). For example, the unpredicted water level increase that appeared along a substantial section of the western Louisiana and northern Texas coasts 12-24 hours in advance of the landfall of Hurricane Ike (in 2008) was identified by Kennedy et al (2011) as a forerunner surge.

The majority of storm surge studies involve numerical modelling. Examples are the Sea, Lake and Overland Surges from Hurricanes model (SLOSH, see

\footnotetext{
${ }^{\star}$ Katrina made first landfall between Hallandale Beach and Aventura on August 25. It made second landfall near Buras-Triumph, Louisiana in early morning of August 29. A few hours later, it made third landfall near the Louisiana-Mississippi border.
} 
Table 4.1: Saffir-Simpson hurricane scale (Simpson, 1974). In grey, the characteristics of Hurricane Katrina (at third landfall) are indicated.

\begin{tabular}{cccc}
\hline $\begin{array}{c}\text { Saffir-Simpson } \\
\text { category }\end{array}$ & $\begin{array}{c}\text { Max wind speed } \\
{\left[\mathrm{m} \mathrm{s}^{-1}\right]}\end{array}$ & $\begin{array}{c}\text { Central pressure* } \\
{[\mathrm{mbar}]}\end{array}$ & $\begin{array}{c}\text { Surge level } \\
{[\mathrm{m}]}\end{array}$ \\
\hline 1 & $33.0-42.5$ & 980 & $1.2-1.5$ \\
2 & $42.9-49.2$ & $965-979$ & $1.8-2.4$ \\
3 & $49.6-58.1$ & $945-964$ & $2.7-3.7$ \\
4 & $58.6-69.3$ & $920-944$ & $4.0-5.5$ \\
5 & $>69.3$ & $<920$ & $>5.5$ \\
\hline C Central pressure & and surge level are estimates based on Saffir-Simpson scale by \\
Simpson (1974).
\end{tabular}

Lin et al, 2010), the ADvanced CIRCulation (ADCIRC, Bunya et al, 2010) coastal circulation and storm surge model and the Japan Meteorological Agency (JMA, Lapidez et al, 2015) storm surge model. These models can provide high resolution predictions but require significant calculating time. For example, using the ADCIRC model, Dietrich et al (2012) simulated the Hurricane Katrina surge on a high performance computing platform. The computed peak water levels are quite accurate, differences with the observations are usually smaller than $0.5 \mathrm{~m}$. However, using 1000 computational cores, the computation still takes 26.7 hours. This property makes it difficult to conduct extensive sensitivity analysis in which the storm characteristics are systematically varied.

The goal of the present study is to investigate the influence of storm characteristics on storm surges in coastal basins such as the New Orleans coastal basin, measured in terms of the set-up at various locations along the coast.

To achieve this goal, we have developed an idealised three-dimensional processbased hydrodynamic model. The model solves the linearised three-dimensional shallow water equations, and includes the Coriolis effect. The atmospheric forcing of the hurricane is represented by the so-called Holland-B model (Holland, 1980). The surge model is fast because it decouples the vertical calculations, which are analytical, from the horizontal calculations, which are carried out using the Finite Element Method.

The model is first applied to simulate the Hurricane Katrina surge of the New Orleans coastal basin in 2005. The modelled area covers the northeastern part of the Gulf of Mexico. Both geometry and topography are schematised. Next, to investigate the influence of storm characteristics, we then perform a sensitivity analysis using a set of synthetic storms. These storms resemble Hurricane Katrina, but we systematically vary the characteristics of the Holland-B model: central pressure, storm size, forward speed, storm direction and landfall point.

This chapter is organised as follows. In $\S 4.2$, we present the model. Next, $\S 4.3$ contains the solution method. The application of the model to Hurricane Katrina surge in the New Orleans coastal basin is presented and discussed in $\S 4.4$. The sensitivity study is presented and discussed in $§ 4.5$. Finally, $\S 4.6$ contains discussions and conclusions. 


\subsection{Model formulation}

\subsubsection{Geometry}

We consider a coastal basin of arbitrary shape and bathymetry on the $f$ plane, with $x$ and $y$ denoting the horizontal coordinates. The vertical coordinate $z$ points upwards, with $z=\eta(x, y, t)$ denoting the free surface elevation with respect to the undisturbed water level $z=0$. The bottom topography $z=$ $-h(x, y)$ varies over the basin. Let $\boldsymbol{u}=(u, v, w)$ represent the flow velocity vector, with components $u, v$ and $w$ in the $x, y$ and $z$-direction, respectively. The domain contains both closed and open boundaries.

\subsubsection{Hydrodynamics}

Assuming that the vertical displacement of the free surface is small compared to the water depth, conservation of momentum and mass is expressed by the three-dimensional linearised shallow water equations on the $f$ plane:

$$
\begin{aligned}
\frac{\partial u}{\partial t}-f v & =-g \frac{\partial \eta}{\partial x}-\rho^{-1} \frac{\partial p_{a}}{\partial x}+K \frac{\partial^{2} u}{\partial z^{2}} \\
\frac{\partial v}{\partial t}+f u & =-g \frac{\partial \eta}{\partial y}-\rho^{-1} \frac{\partial p_{a}}{\partial y}+K \frac{\partial^{2} v}{\partial z^{2}} \\
\frac{\partial u}{\partial x}+\frac{\partial v}{\partial y}+\frac{\partial w}{\partial z} & =0 .
\end{aligned}
$$

Here, $f=2 \Omega \sin \vartheta$ is the Coriolis parameter (with $\Omega=7.292 \times 10^{-5} \mathrm{rad} \mathrm{s}^{-1}$ the angular frequency of the Earth's rotation and $\vartheta$ the latitude), $g=9.81 \mathrm{~m} \mathrm{~s}^{-2}$ the gravitational acceleration, $\rho=1.0 \times 10^{3} \mathrm{~kg} \mathrm{~m}^{-3}$ the density of water and $p_{a}(x, y, t)$ the atmospheric pressure. Turbulence is represented using vertical eddy viscosity $K$ (spatially uniform and constant in time), combined with a partial slip condition at the bed. Horizontal mixing of momentum is neglected.

The kinematic and dynamic boundary conditions at the surface and bottom read, in linearised form:

$$
\begin{array}{ll}
w=\frac{\partial \eta}{\partial t}, \quad K\left(\frac{\partial u}{\partial z}, \frac{\partial v}{\partial z}\right)=\frac{\left(\tau_{\mathrm{w}}^{(x)}, \tau_{\mathrm{w}}^{(y)}\right)}{\rho} & \text { at } z=0, \\
w=0, \quad K\left(\frac{\partial u}{\partial z}, \frac{\partial v}{\partial z}\right)=s(u, v) & \text { at } z=-h,
\end{array}
$$

where $s$ is the resistance parameter (also spatially uniform and constant in time). The linearisation procedure implies that the free surface condition in Eq.(4.4) is imposed at $z=0$ instead of at $z=\eta$. Furthermore, $\tau_{\mathrm{w}}=\left(\tau_{\mathrm{w}}^{(x)}, \tau_{\mathrm{w}}^{(y)}\right)$ is the wind stress vector, with components in $x$ - and $y$ direction. The wind stress and the atmospheric pressure $p_{a}$ will be specified in $\S 4.2 .3$.

At the closed horizontal boundaries, we require zero normal transports, i.e.

$$
\left[\int_{-h}^{0}(u, v) \mathrm{d} z\right] \cdot \boldsymbol{n}=0 .
$$



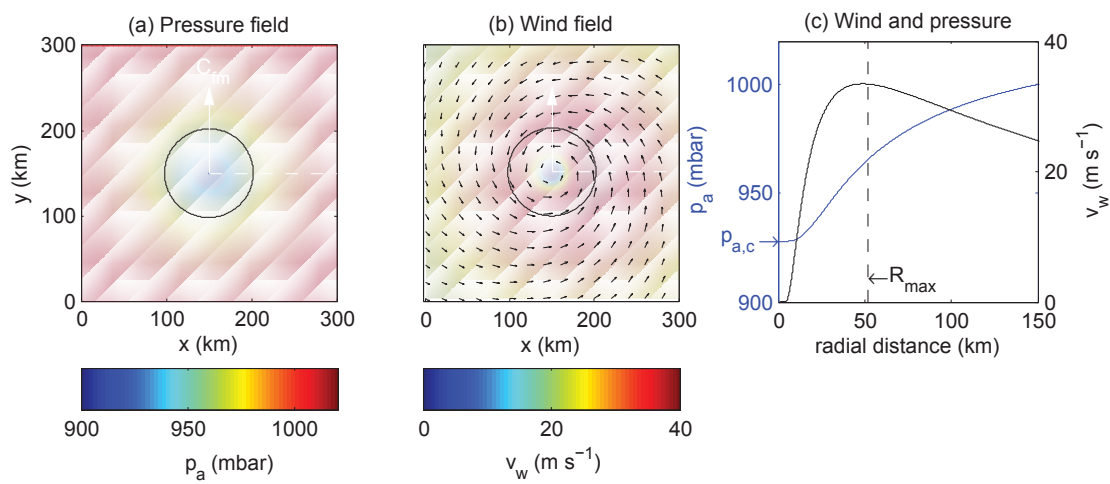

Figure 4.1: Example of atmospheric forcing due to Holland-B model, showing 2Dplots of $(a)$ pressure, $(b)$ wind field as well as $(c)$ radial profiles of pressure and wind (along the white dashed line in $a$ and $b$ ). Model parameters: $p_{a, c}=928$ mbar, $p_{a, \infty}=1030$ mbar, $R_{\max }=52 \mathrm{~km}$ (black circle in $a$ and $\left.b\right), B=1$ and $C_{\mathrm{fm}}=7.8 \mathrm{~m} \mathrm{~s}^{-1}$ (moving north, so $\phi=0^{\circ}$ ).

where $\boldsymbol{n}=\left(n_{x}, n_{y}\right)$ is the local outward pointing normal vector. At the open boundaries, the sea surface elevation is calculated from the inverted barometer effect (Doodson, 1924), i.e.

$$
\eta=\frac{p_{a, \infty}-p_{a}}{\rho g}
$$

where $p_{a, \infty}$ is ambient pressure.

\subsubsection{Atmospheric forcing}

The atmospheric forcing of a storm is described by the so-called Holland-B model (Holland, 1980), a model widely used for its simplicity and flexibility in radial structure (Madsen and Jakobsen, 2004). The surface pressure $p_{a}$ at a distance $r$ from the storm centre is modelled as:

$$
p_{a}(r)=p_{a, c}+\left(p_{a, \infty}-p_{a, c}\right) \exp \left(-\left(R_{\max } / r\right)^{B}\right)
$$

where $p_{a, c}$ is the atmospheric pressure at the hurricane centre, $p_{a, \infty}$ the ambient pressure, and $B$ the so-called Holland-B parameter that describes the peakedness of the pressure profile. An example of the pressure field is given in Figure 4.1a. The wind field is then calculated as a so-called gradient wind, i.e. from a balance between the Coriolis force and the pressure gradient (Fig.4.1b). Blaton's adjustment of the radius of curvature (Pita et al, 2012) is included to capture the asymmetry of the wind field introduced by the forward movement of the hurricane. The magnitude of surface wind velocity $v_{\mathrm{w}}(r)$ (at $10 \mathrm{~m}$ above water surface) at a distance $r$ from the centre is given by 


$$
v_{\mathrm{w}}(r)=\frac{2}{3}\left(-W+\sqrt{W^{2}+\frac{\left(p_{a, \infty}-p_{a, c}\right) B}{\rho_{\text {air }}}\left(\frac{R_{\max }}{r}\right)^{B} \exp \left(-\left(\frac{R_{\max }}{r}\right)^{B}\right)}\right),
$$

with

$$
W=\frac{C_{\mathrm{fm}} \sin \xi+r f}{2},
$$

where $\rho_{\text {air }}=1.225 \mathrm{~kg} \mathrm{~m}^{-3}$ is the air density. In Eq.(4.9), the factor $2 / 3 \mathrm{ac}$ counts for the deceleration by the water surface. Furthermore, the direction of the wind velocity is $17^{\circ}$ deflected from the isobar towards the lower pressure (counterclockwise) (Klaver et al, 2006). At a distance $R_{\max }$ away from the centre, wind reaches its maximum speed, see Figure $4.1 b$ and $4.1 c$. The hurricane moves forward with speed $C_{\mathrm{fm}} ; \xi$ in Eq.(4.9) is then the angle between the direction of storm translation $(\phi$, measured clockwise with respect to the north direction) and the vector from the storm centre to the point of calculation. Storm parameters are fixed in the sensitivity analysis but allowed to evolve over time when simulating Hurricane Katrina's surge. Hurricane Katrina followed a curved track, resulting in time-varying parameters.

The wind stress is estimated through the wind-shear formula of Smith et al (1992),

$$
\boldsymbol{\tau}_{\mathrm{w}}=C_{\mathrm{d}} \rho_{\mathrm{air}}\left|\boldsymbol{v}_{\mathrm{w}}\right| \boldsymbol{v}_{\mathrm{w}} .
$$

where $C_{\mathrm{d}}=2.5 \times 10^{-3}$ is the wind drag coefficient, as suggested by Resio and Westerink (2008) for the region of the strongest surge generation in hurricanes.

\subsection{Solution method}

Our solution method, as sketched in Fig. 4.2, makes use of the frequency domain. To this end, the time-dependent wind stress and pressure field are written as a superposition of time-periodic signals at different frequencies through a discrete Fourier transformation. We then obtain the solution at each frequency. The actual surge level is then the superposition of the surge level at all frequencies.

\subsubsection{Fourier expansion of the problem}

We apply a discrete Fourier transformation to the wind stress and atmospheric pressure. This means that the continuous signal is decomposed in a number of signals (modes indicated with index $m$ ) with different frequencies $\left(\omega_{m}\right)$, i.e.

$$
\left(\frac{\tau_{\mathrm{w}}^{(x)}}{\rho}, \frac{\tau_{\mathrm{w}}^{(y)}}{\rho}, p_{a}\right)=\Re\left[\sum_{m=-M}^{M}\left(T_{m}^{(x)}(x, y), T_{m}^{(y)}(x, y), P_{a, m}(x, y)\right) \exp \left(-\mathrm{i} \omega_{m} t\right)\right]
$$




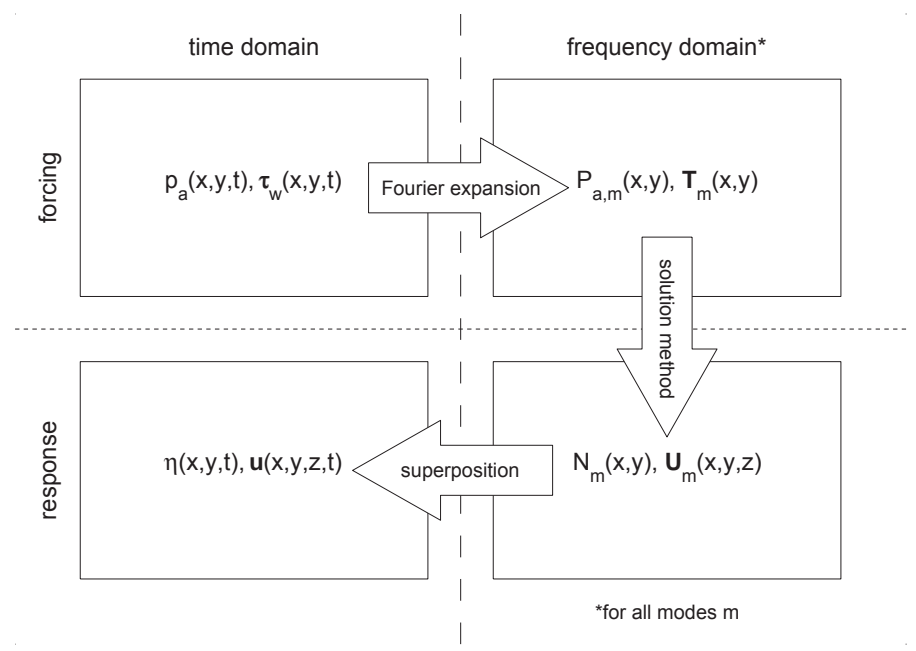

Figure 4.2: Sketch of the solution method. The forcing, specified in the time domain, is transformed to the frequency domain in which the response is obtained using the FEM-model. The superposition of the solutions at all frequencies then provides the solution in the time domain.

where $\Re$ denotes the real part and $M$ is the truncation number. Furthermore, $T_{m}^{(x)}, T_{m}^{(y)}$ and $P_{a, m}$ are the complex amplitudes ${ }^{\dagger}$ of the wind stress and the pressure at frequency $\omega_{m}$, with $\omega_{m}=m \omega_{\min }$. Here $\omega_{\min }=2 \pi / T_{\text {recur }}$ is the lowest frequency which results from a choice of recurrence time $T_{\text {recur. }}$ As a consequence of the discrete Fourier expansion, the storm event will recur after a period of $T_{\text {recur }}$, as shown in Figure 4.3. To avoid interference of subsequent events, this recurrence period should be long enough. At the same time, $M$ should be chosen sufficiently large, because $2 \pi / \omega_{\max }=T_{\text {recur }} / M$ determines the temporal resolution of the transformed forcing signals.

Next, we write the solution in the same fashion as Eq. (4.12), i.e. for the surface elevation

$$
\eta(x, y, t)=\Re\left[\sum_{m=-M}^{M} N_{m}(x, y) \exp \left(-\mathrm{i} \omega_{m} t\right)\right],
$$

with complex elevation amplitudes $N_{m}(x, y)$. Similar expressions hold for $u, v$ and $w$, with complex amplitudes $U_{m}(x, y), V_{m}(x, y)$ and $W_{m}(x, y)$.

\footnotetext{
${ }^{\dagger}$ Because the forcing is real-valued, it follows that $T_{-m}^{(x)}=\bar{T}_{m}^{(x)}$ in Eq.(4.12), with an overbar denoting complex conjugation (the same applies to the other amplitudes).
} 


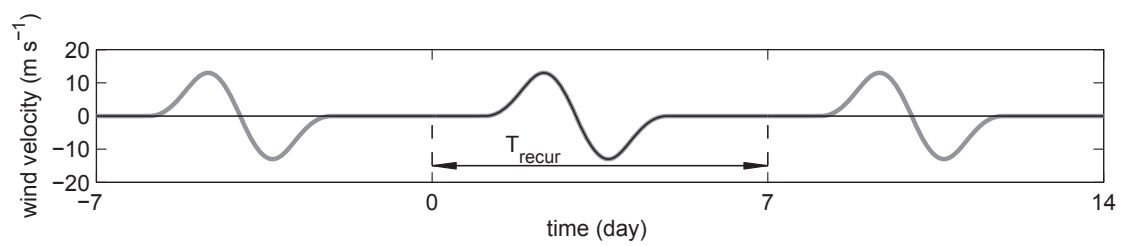

Figure 4.3: Example of a wind event (wind velocity as a function time, thin black line), which - as a result of our Fourier expansion - transforms to a periodic signal (thick grey line). This periodic signal recurs over a period $T_{\text {recur }}=2 \pi / \omega_{\min }$, here chosen equal to 7 days.

\subsubsection{Differential problem for surface elevation amplitude at each frequency}

To derive a differential problem for the surface elevation, we first turn to the vertical profile of the horizontal flow. At each frequency $\omega_{m}$, the (complex) amplitudes of the horizontal flow components $U_{m}$ and $V_{m}$ are written as the superposition of three terms:

- one term proportional to the amplitude of the wind stress $\boldsymbol{T}_{m}$,

- a second term proportional to the amplitude of the atmospheric pressure gradient $\nabla P_{a, m}$,

- a third term proportional to the gradient of the surface elevation amplitude $\nabla N_{m}$.

The vertical profiles of these terms are derived using so-called rotating flow components, see Appendix 4.A. This makes the first two contributions above known. However, the gradient of the surface elevation $\nabla N_{m}$ is still unknown. It follows from solving an elliptical problem for $N_{m}$. This problem is obtained by substituting the above expressions into the continuity equation and integrating from bottom to surface (denoted by $\langle\cdot\rangle)$ :

$$
\nabla_{\mathrm{h}} \cdot\left[\left\langle\mathbf{D}_{m}\right\rangle \nabla_{\mathrm{h}} N_{m}\right]-\mathrm{i} \omega_{m} N_{m}=-\nabla_{\mathrm{h}} \cdot\left\langle\mathbf{r}_{m}\right\rangle .
$$

Here we have introduced the horizontal nabla operator $\nabla_{\mathbf{h}}=(\partial / \partial x, \partial / \partial y)^{\mathrm{T}}$ as well as $2 \times 2$-matrix $\mathbf{D}_{m}$ and $2 \times 1$-vector $\mathbf{r}_{m}$, given by

$$
\mathbf{D}_{m}=\left[\begin{array}{cc}
C_{m}^{+} & C_{m}^{-} \\
-C_{m}^{-} & C_{m}^{+}
\end{array}\right], \quad \mathbf{r}_{m}=\left[\begin{array}{c}
R_{m}^{+} \\
R_{m}^{-}
\end{array}\right] .
$$

The coefficients $C_{m}^{+}$and $C_{m}^{-}$as well as the forcing terms $R_{m}^{+}$and $R_{m}^{-}$depend on topography and thus on $x$ and $y$; they are specified in Appendix 4.A.

The no-normal transport conditions in Eq.(4.6) at the closed boundaries imply

$$
\left(\left\langle\mathbf{D}_{m}\right\rangle \boldsymbol{\nabla}_{\mathrm{h}} N_{m}+\left\langle\mathbf{r}_{m}\right\rangle\right) \cdot \boldsymbol{n}=0 .
$$


Furthermore, according to Eq.(4.7), the amplitudes $N_{m}$ along the open boundary are given by

$$
\begin{array}{ll}
N_{m}=\frac{P_{a, 0}-p_{a, \infty}}{\rho g}, & \text { for } m=0, \\
N_{m}=\frac{P_{a, m}}{\rho g}, & \text { for } m \neq 0 .
\end{array}
$$

Finally, the vertical flow amplitude $W_{m}$ at any vertical position $z$ can be expressed in terms of gradient of the free surface elevation $N_{m}$ and its gradient, as well as forcings $\boldsymbol{T}_{m}$ and $\boldsymbol{\nabla} P_{a, m}$. This follows from vertical integration of the continuity equation (4.3), using the boundary conditions in Eq.(4.4)-(4.5), see Appendix 4.A.3.

\subsubsection{Finite Element Method}

The problem in Eqs.(4.14)-(4.17) for the elevation amplitude $N_{m}$ is solved by applying the Finite Element Method (FEM). To this end, the coastal basin is discretised into a set of $P$ triangular elements. The solution is then written as

$$
N_{m}(x, y)=\sum_{p=1}^{P} N_{m, p} \phi_{p}(x, y),
$$

with coefficients $N_{m, p}$ and basis functions $\phi_{p}(x, y)$. To obtain the coefficients $N_{m, p}$, Eq.(4.14) is first cast in weak form, which involves multiplication with a test function and integration over the domain. Details of this procedure can be found in Gockenbach (2006), see also Kumar et al (submitted), who developed a similar model to study tidal dynamics in estuaries.

\subsection{Modelling the Hurricane Katrina surge}

In this section, we apply our model to simulate the surge levels in the New Orleans coastal basin induced by Hurricane Katrina in 2005. The goal of this case study is to gain confidence in the model by reproducing the qualitative behavior of the surge as well as the magnitude of the surge levels, before performing the sensitivity analysis with synthetic storms. We schematize the geometry and bathymetry of the New Orleans coastal basin, which is presented in $\S 4.4 .1$. The wind and pressure fields of Hurricane Katrina are modelled using the Holland-B model; a brief description on the choices of storm parameters is given in §4.4.2. The hydrodynamic parameters and numerical settings are presented in $§ 4.4 .3$. Finally, in $\S 4.4 .4$, the model results are presented and compared against observations (National Oceanic and Atmospheric Administration, 2015) and simulations using the ADCIRC model (Dietrich et al, 2012).

\subsubsection{Geometry and bathymetry}

We consider a domain that covers the northeastern corner of the Gulf of Mexico (Figure 4.4), since Blain et al (1994) argued that large-scale processes are important. As a further schematization, the coastlines of the New Orleans coastal 


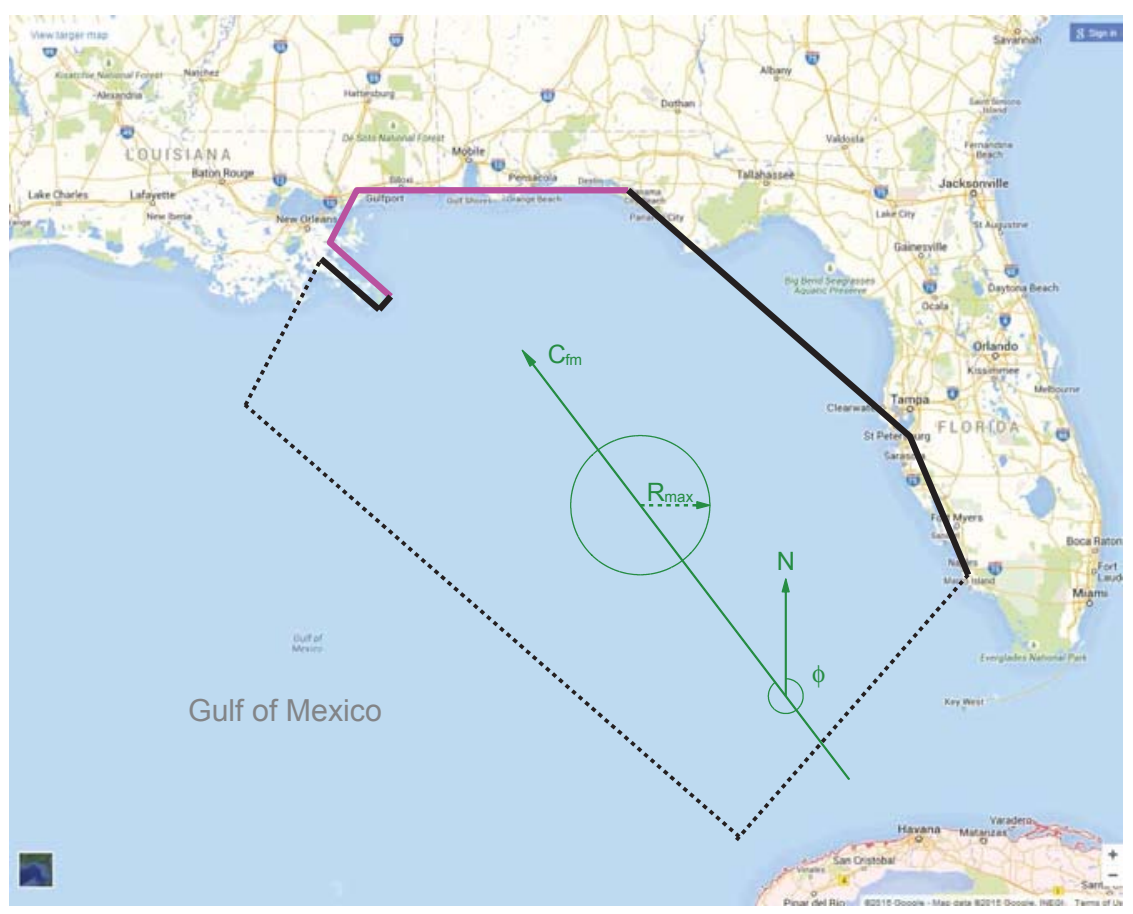

Figure 4.4: Schematised model domain of the north east corner of the Gulf of Mexico, where pink lines indicate the New Orleans coastal area, black dashed lines the open boundaries and black lines the other domain boundaries. The schematic representation of a storm and storm track are plotted in green. 


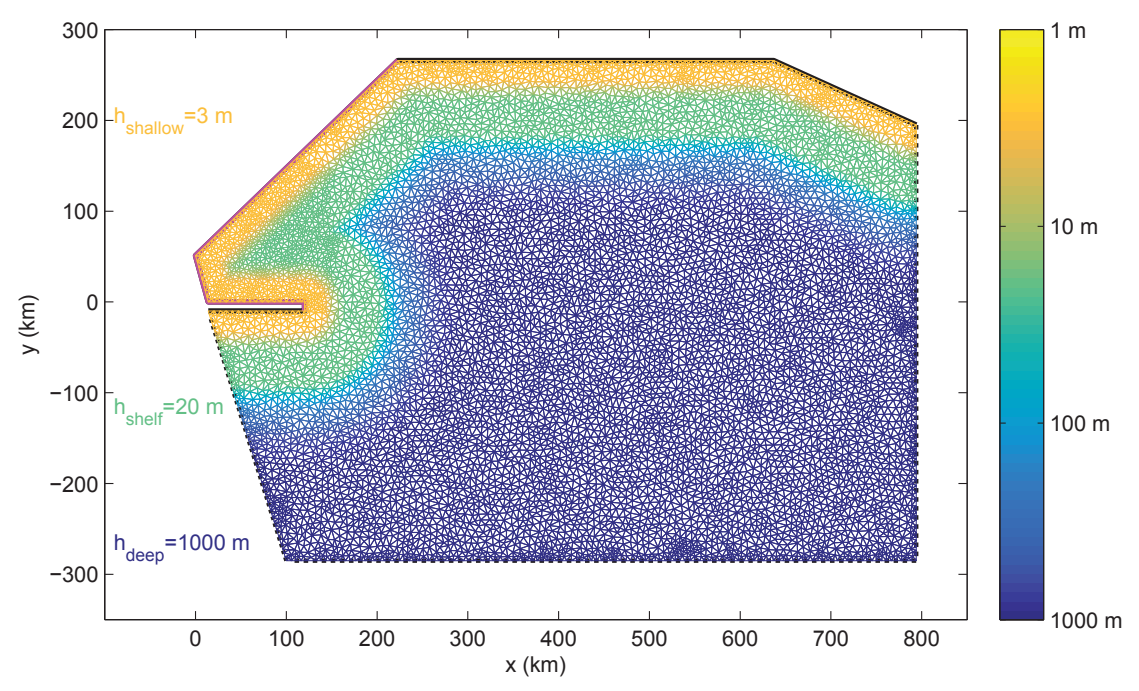

Figure 4.5: Model domain, showing mesh and bathymetry ranging from $h_{\text {shallow }}=3$ $\mathrm{m}$ to $h_{\text {shelf }}=20 \mathrm{~m}$ and $h_{\text {deep }}=1000 \mathrm{~m}$. Depth colours are plotted in a log scale, color of boundaries as in Fig. 4.4.

basin are represented by straight lines, including the Mississippi dike. The basin is located at an average latitude of $29^{\circ} N$, which implies $f=7.07 \times 10^{-5} \mathrm{rad} \mathrm{s}^{-1}$.

The New Orleans coastal basin is situated at a continental shelf, for which we adopt a simplified bathymetry. The water depth at a given location is defined as a function of distance to the coast. As shown in Figure 4.5, it increases from $h_{\text {shallow }}=3 \mathrm{~m}$ near the coast to $h_{\text {shelf }}=20 \mathrm{~m}$ on the shelf, and further to $h_{\text {deep }}=1000 \mathrm{~m}$ in the offshore part of the Gulf of Mexico. This leads to a bathymetry with depth contours roughly parallel to the coastline.

\subsubsection{Derivation of storm parameters}

Hurricane Katrina followed a curved track (denoted by red arrow in Figure 4.6) with varying characteristics over time. To extract the parameters of Hurricane Katrina, we use the 6-hourly data HURDAT2 (Hurricane Database2, National Climatic Data Center, 2014) of the central pressure and location of the hurricane centre provided by NOAA. This information is used to obtain the forward speed $\left(C_{\mathrm{fm}}\right)$ and storm track $(\phi)$. In addition, we use a 3 -hourly maximum radius $R_{\max }$ and Holland-B parameter $B$ from measured wind speeds and pressure profiles of the hurricane, as derived by Vickery and Wadhera (2008). Figure 4.7 shows the storm parameters as a function of time. Between the raw data points, the parameters have been linearly interpolated. 


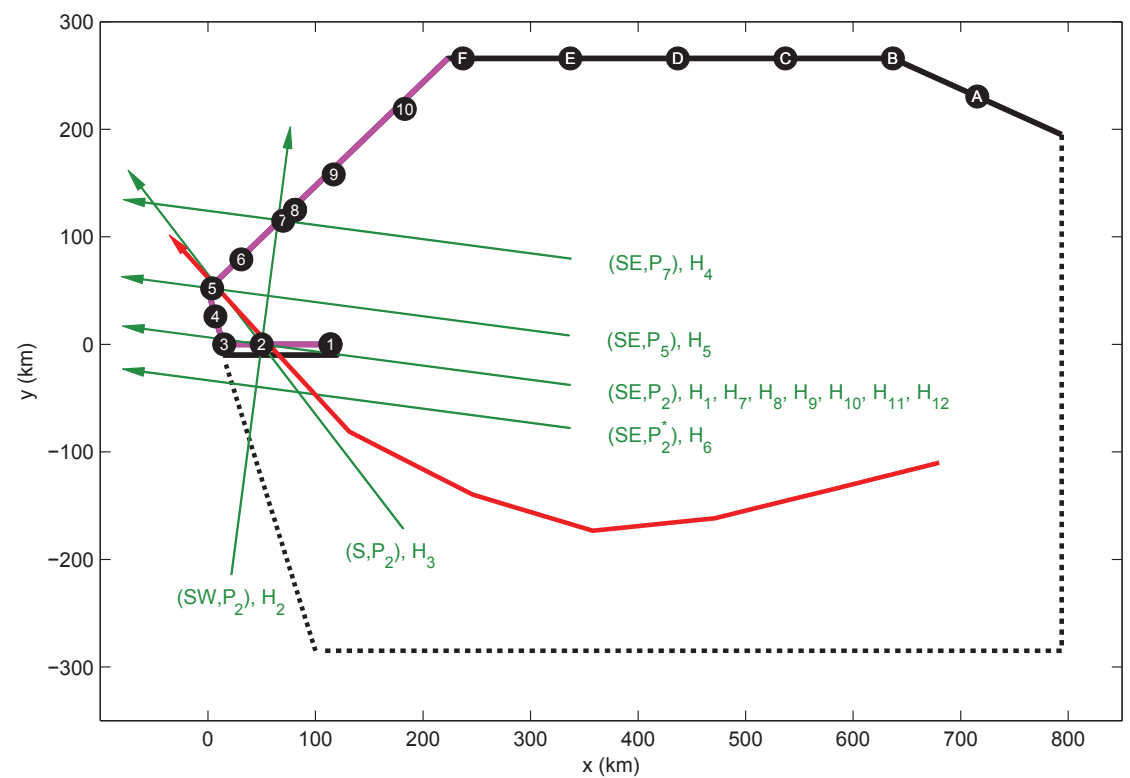

Figure 4.6: Model domain showing the observation points $P_{1}-P_{10}$ listed in Table 4.3 as numbered black dots. The curved red arrow indicates the track of Hurricane Katrina. Also shown are the seven different storm tracks (green) and the corresponding synthetic storms $H_{j}$ listed in Table 4.4, used in the sensitivity analysis. The tracks are characterised by approaching direction (SW, S or SE) and landfall point $P_{j}$. The points $\mathrm{A}-\mathrm{F}$ are used in the forerunner analysis ( $\$ 4.5 .7)$.

Table 4.2: Overview of default model parameters and numerical choices.

\begin{tabular}{lccc}
\hline Description & Symbol & Value & Unit \\
\hline Latitude & $\vartheta$ & 29 & ${ }^{\circ} \mathrm{N}$ \\
Coriolis parameter & $f$ & $7.07 \times 10^{-5}$ & $\mathrm{rad} \mathrm{s}^{-1}$ \\
Coastal water depth & $h_{\text {shallow }}$ & 3 & $\mathrm{~m}$ \\
Deep water depth & $h_{\text {deep }}$ & 1000 & $\mathrm{~m}$ \\
Vertical eddy viscosity & $K$ & 0.01 & $\mathrm{~m}^{2} \mathrm{~s}^{-1}$ \\
Resistance parameter & $s$ & 0.015 & $\mathrm{~m} \mathrm{~s}^{-1}$ \\
Fourier truncation number & $M$ & 64 & - \\
Recurrence period & $T_{\text {recur }}$ & 7 & day \\
Number of nodes & $P$ & 35748 & - \\
\hline
\end{tabular}


(a) Central pressure

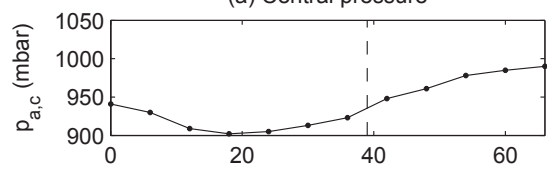

(c) Holland-B parameter

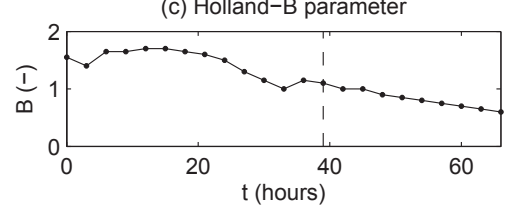

(b) Radius to maximum winds

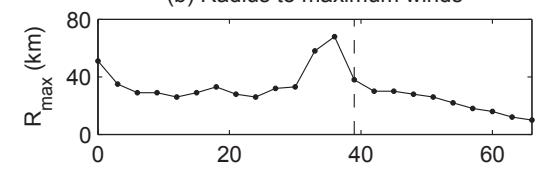

(d) Forward speed

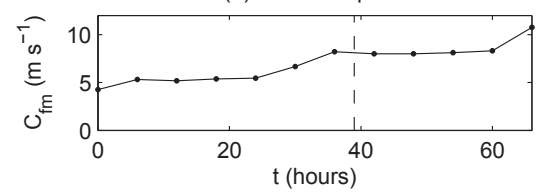

Figure 4.7: The evolution of storm parameters of Hurricane Katrina (2005) from 28-8-2005 0.00h ( $t=0$ hours) until 30-8-2005 18.00h. The black dots indicate the raw data from HURDAT2 (Hurricane Database2, National Climatic Data Center, 2014) and Vickery and Wadhera (2008).

Table 4.3: Maximum surge levels observed (National Oceanic and Atmospheric Administration, 2015) or simulated using the ADCIRC model (Dietrich et al, 2012) for Hurricane Katrina.

\begin{tabular}{clcc}
\hline Location $^{\dagger}$ & Station name & $\begin{array}{c}\text { observations } \\
\text { NOAA }^{\ddagger}[\mathrm{m}]\end{array}$ & $\begin{array}{c}\text { simulated } \\
\text { ADCIRC }[\mathrm{m}]\end{array}$ \\
\hline$P_{1}$ & SW Pass, LA (8760922) & 2.4 & 3.3 \\
$P_{2}$ & - & - & 5.2 \\
$P_{3}$ & - & - & 4.5 \\
$P_{4}$ & - & - & 4.7 \\
$P_{5}$ & - & - & 6.2 \\
$P_{6}$ & Waveland, MS (8747766) & $2.7^{*}$ & 7.8 \\
$P_{7}$ & Ocean springs, MS (8743281) & $4.0^{*}$ & - \\
$P_{8}$ & Horn Island, MS (8742221) & $1.9^{*}$ & 4.1 \\
$P_{9}$ & Dauphin Isl. AL (8735180) & 1.9 & 2.5 \\
$P_{10}$ & Pensacola, FL (8729840) & 2.0 & - \\
\hline
\end{tabular}

${ }^{\dagger}$ The locations $P_{j}$ are shown in Figure 4.6.

${ }^{\ddagger}$ Relative to NAVD88 (2004.65).

* maximum value before sensor ceased transmission (true maximum not recorded). 


\subsubsection{Hydrodynamic parameters and numerical setting}

An overview of default model parameters and numerical choices is provided in Table 4.2. In this study, we take a vertical eddy viscosity of $K=0.01 \mathrm{~m}^{2} \mathrm{~s}^{-1}$ and a resistance parameter of $s=0.015 \mathrm{~m} \mathrm{~s}^{-1}$. These values are obtained by tuning the model to the measured data.

The model domain is discretised into a set of 35748 unstructured triangles, with a grid size of $8 \mathrm{~km}$ in the offshore part and $4 \mathrm{~km}$ in the coastal area (see Fig. 4.5). The grid sizes have been determined through a convergence test presented in Appendix 4.B, reflecting a trade-off between accuracy and computation time. Regarding the time resolution, we employ 65 modes $(M=$ 64) accompanied with a recurrence period of $T_{\text {recur }}=7$ days. As demonstrated in Appendix 4.C, this choice gives an accurate representation of the forcings of Hurricane Katrina, while keeping the calculation time acceptable for the purpose of the sensitivity study.

\subsubsection{Model results}

The water levels simulated by our model are evaluated at 10 locations (see Table 4.3 and Figure 4.6) and presented in Figure 4.8. This figure also displays the observations and the ADCIRC-results. Note that observations are incomplete, as some sensors ceased transmission during the event. The ADCIRC results are generally within $0.5 \mathrm{~m}$ difference from the observations, except at $P_{1}$.

Qualitatively, the model results are good, capturing the temporal and spatial surge pattern as well as reproducing the timing of the peak values. An exception is location $P_{1}$ (the tip of the Mississippi dike), where observed surges are positive but our model results display negative surges around the moment of landfall. This discrepancy can be due to overflow of the Mississippi dike, which is not incorporated in our model.

Quantitatively, the magnitude of the peak levels is relatively well reproduced. Differences with the ADCIRC-simulations are less than $1 \mathrm{~m}$, except at $P_{5}$. Our model underestimates the peak values in the area close to the Mississippi dike $\left(P_{2}, P_{3}, P_{4}, P_{5}\right)$, whereas it overestimates the peak values further to the east $\left(P_{8}, P_{9}, P_{10}\right)$.

We also investigated the sensitivity of the model results to the friction parameters (vertical eddy viscosity $K$ and slip parameter $s$ ) and bathymetry. Results show that lower values of $K$ and $s$ lead to slightly higher water level, but the difference is small. Regarding bathymetry, three values are taken for the water depth at the coast, $h_{\text {shallow }}=3 \mathrm{~m}, 5 \mathrm{~m}$ and $7 \mathrm{~m}$, whereas the depth values in the shelf and offshore part are kept the same: $h_{\text {shelf }}=20 \mathrm{~m}$ and $h_{\text {deep }}=1000$ $\mathrm{m}$. The comparison shows that the surge event with $h_{\text {shallow }}=3 \mathrm{~m}$ provides the best fit with the observation and ADCIRC results. Simulated water levels with $h_{\text {shallow }}=5 \mathrm{~m}$ and $h_{\text {shallow }}=7 \mathrm{~m}$ have higher peak surges and stronger oscillation after the storm landfalling near the coast, because there is less dissipation.

In view of the schematizations, we conclude that our model qualitatively captures the Katrina surge in the New Orleans coastal basin and also produces 

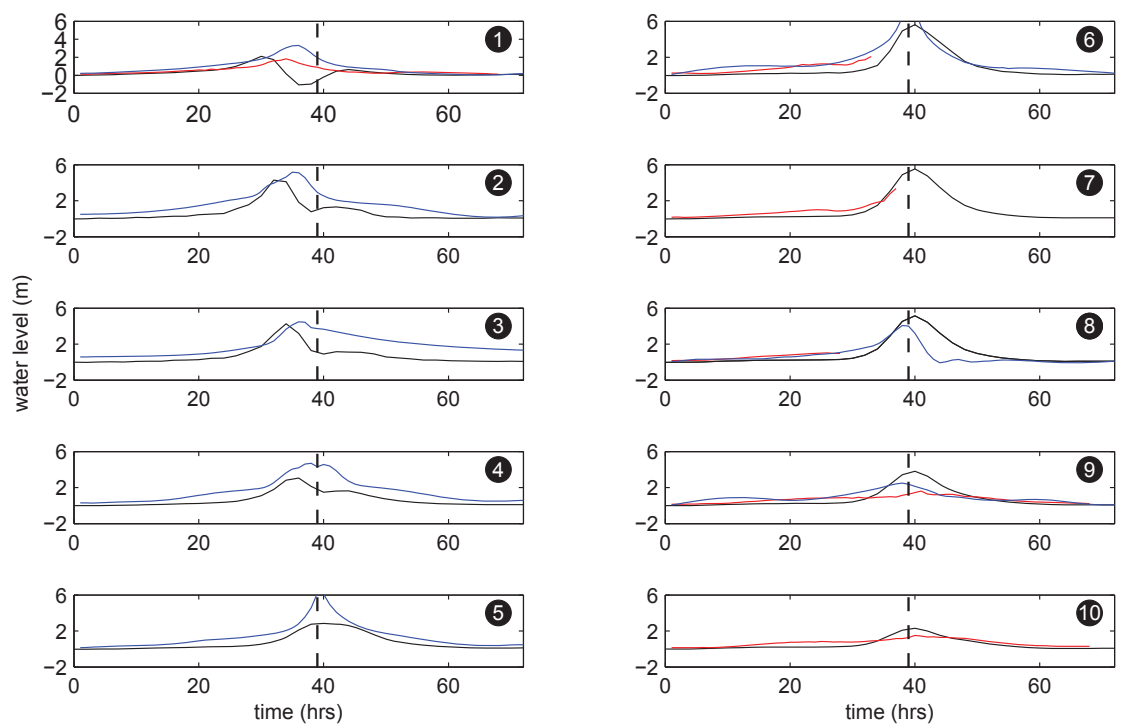

New model — Observed $\longrightarrow$ ADCIRC

Figure 4.8: Comparison between new model results (black line) and observations (red) and ADCIRC simulations (blue) at locations $P_{1}$ to $P_{10}$. Detailed information of the stations is stated in Table 4.3 and shown in Figure 4.6. The time starts at 28-8$20050.00 \mathrm{~h}$, and the black dashed line denotes the third landfall time of Hurricane Katrina.

similar quantitative results. Hence, the model can be used to study the sensitivity of surge levels to storm parameters.

\subsection{Sensitivity of surge levels to storm characteristics}

To investigate the sensitivity of surge levels to storm characteristics, we design a set of synthetic storms with different parameters. In $\S 4.5 .1$, scenarios with varying storm direction $(\phi)$, point of landfall, storm size $\left(R_{\max }\right)$, forward speed $\left(C_{\mathrm{fm}}\right)$ and central pressure $\left(p_{a, c}\right)$ are presented. The sensitivity of surges to storm parameters is presented and discussed in the subsequent sections, §4.5.2$\S 4.5 .5$. Finally, in $\S 4.5 .7$ and $\S 4.5 .8$, we study the possible forerunner surge and the Fourier spectrum of the elevation amplitude, respectively.

\subsubsection{Synthetic storms}

We consider 12 hurricane scenarios (see Table 4.4). These hurricanes making landfall near New Orleans are mainly from the southern, southwestern and southeastern directions. We first introduce a reference storm scenario $H_{1}$, approaching the New Orleans coastal basin from a southeastern direction 
Table 4.4: Properties of 13 synthetic storms

\begin{tabular}{llccccccc}
\hline \multicolumn{2}{c}{ Scenario $^{\S}$} & Dir. $^{\dagger}$ & $\begin{array}{c}\text { Landfall } \\
\text { Point }\end{array}$ & $\begin{array}{c}R_{\max } \\
{[\mathrm{km}]}\end{array}$ & $\begin{array}{c}C_{\mathrm{fm}} \\
{\left[\mathrm{m} \mathrm{s}^{-1}\right]}\end{array}$ & $\begin{array}{c}p_{a, c} \\
{[\mathrm{mbar}]}\end{array}$ & $\begin{array}{c}B \\
{[-]}\end{array}$ & Category $^{\ddagger}$ \\
\hline$H_{1}$ & $\left(\mathrm{SE}, P_{2}\right)_{\text {ref }}$ & $\mathrm{SE}$ & $P_{2}$ & 50 & 6 & 900 & 1.5 & 3 \\
$H_{2}$ & $\left(\mathrm{SW}, P_{2}\right)$ & $\mathrm{SW}$ & $P_{2}$ & 50 & 6 & 900 & 1.5 & 3 \\
$H_{3}$ & $\left(\mathrm{~S}, P_{2}\right)$ & $\mathrm{S}$ & $P_{2}$ & 50 & 6 & 900 & 1.5 & 3 \\
$H_{4}$ & $\left(\mathrm{SE}, P_{7}\right)$ & $\mathrm{SE}$ & $P_{7}$ & 50 & 6 & 900 & 1.5 & 3 \\
$H_{5}$ & $\left(\mathrm{SE}, P_{5}\right)$ & $\mathrm{SE}$ & $P_{5}$ & 50 & 6 & 900 & 1.5 & 3 \\
$H_{6}$ & $\left(\mathrm{SE}, P_{2}^{*}\right)$ & $\mathrm{SE}$ & $P_{2}^{*}$ & 50 & 6 & 900 & 1.5 & 3 \\
$H_{7}$ & (smaller) & $\mathrm{SE}$ & $P_{2}$ & 30 & 6 & 900 & 1.5 & 3 \\
$H_{8}$ & (larger) & $\mathrm{SE}$ & $P_{2}$ & 70 & 6 & 900 & 1.5 & 3 \\
$H_{9}$ & (slower) & $\mathrm{SE}$ & $P_{2}$ & 50 & 3 & 900 & 1.5 & 3 \\
$H_{10}$ & (faster) & $\mathrm{SE}$ & $P_{2}$ & 50 & 10 & 900 & 1.5 & 3 \\
$H_{11}$ & (weaker) & $\mathrm{SE}$ & $P_{2}$ & 50 & 6 & 930 & 1.5 & 2 \\
$H_{12}$ & (stronger) & $\mathrm{SE}$ & $P_{2}$ & 50 & 6 & 870 & 1.5 & 4 \\
\hline
\end{tabular}

${ }^{\S}$ Bracketed description shows the difference with the reference storm $H_{1}$.

${ }^{\dagger}$ Storm direction: SW means $\phi=7.5^{\circ}$, S means $\phi=322.5^{\circ}$ and SE means $\phi=277.5^{\circ}$. ${ }^{\ddagger}$ According to the Saffir-Simpson scale (Table 4.1).

${ }^{*}$ The landfall location $P_{2}^{*}$ is $45 \mathrm{~km}$ south of location $P_{2}$.

$\left(\phi=277.5^{\circ}\right)$ and making landfall at location $P_{2}$ with $R_{\max }=50 \mathrm{~km}, p_{a, c}=900$ mbar and $C_{\mathrm{fm}}=6 \mathrm{~m} \mathrm{~s}^{-1}$. The values of these parameters are representative for Hurricane Katrina. Although the straight track of $H_{1}$ does not precisely follow the curved track of Hurricane Katrina (red curve in Fig. 4.6), it captures most features of the track, including the landfall point. Storms $H_{4}, H_{5}$ and $H_{6}$ also approach from a southeastern direction but differ regarding landfall location. Alternatively, storms $H_{3}$ approaches from a southern direction $\left(\phi=322.5^{\circ}\right)$ and storm $H_{2}$ from southwestern direction $\left(\phi=7.5^{\circ}\right)$. The six tracks introduced here are presented in Figure 4.6 (green arrows). Storms $H_{7}$ and $H_{8}$ follow the same track as $H_{1}$, but with radii to maximum wind of $R_{\max }=30 \mathrm{~km}$ and 70 $\mathrm{km}$, respectively. The storm centres of scenarios $H_{9}$ and $H_{10}$ are identical to the reference scenario $H_{1}$, but they move slower $\left(C_{\mathrm{fm}}=3 \mathrm{~m} \mathrm{~s}^{-1}\right)$ and faster $\left(C_{\mathrm{fm}}=10 \mathrm{~m} \mathrm{~s}^{-1}\right)$, respectively. Finally, the central pressures of storms $H_{11}$ and $H_{12}$ are $p_{a, c}=930 \mathrm{mbar}$ and $p_{a, c}=870 \mathrm{mbar}$, respectively. Peak surges observed at the selected locations $\left(P_{j}\right.$, for $\left.j=1,2, \cdots, 10\right)$ for each of the 12 storm scenarios are summarised in Table 4.5.

\subsubsection{Sensitivity to storm direction}

We now consider storms from the three directions mentioned in $\S 4.5 .1$ and shown in Fig 4.6. Storm $H_{1}$ approaches from the southeastern direction $\left(\phi=277.5^{\circ}\right)$. When it moves into the basin, the winds at the mouth of the basin are blowing into the basin. It makes second landfall between $P_{3}$ and $P_{4}$, with maximum winds blowing onshore at $P_{5}$. Storm $H_{2}$ approaches the New Orleans coastal basin from the southwestern direction $\left(\phi=7.5^{\circ}\right)$. When the storm makes first 

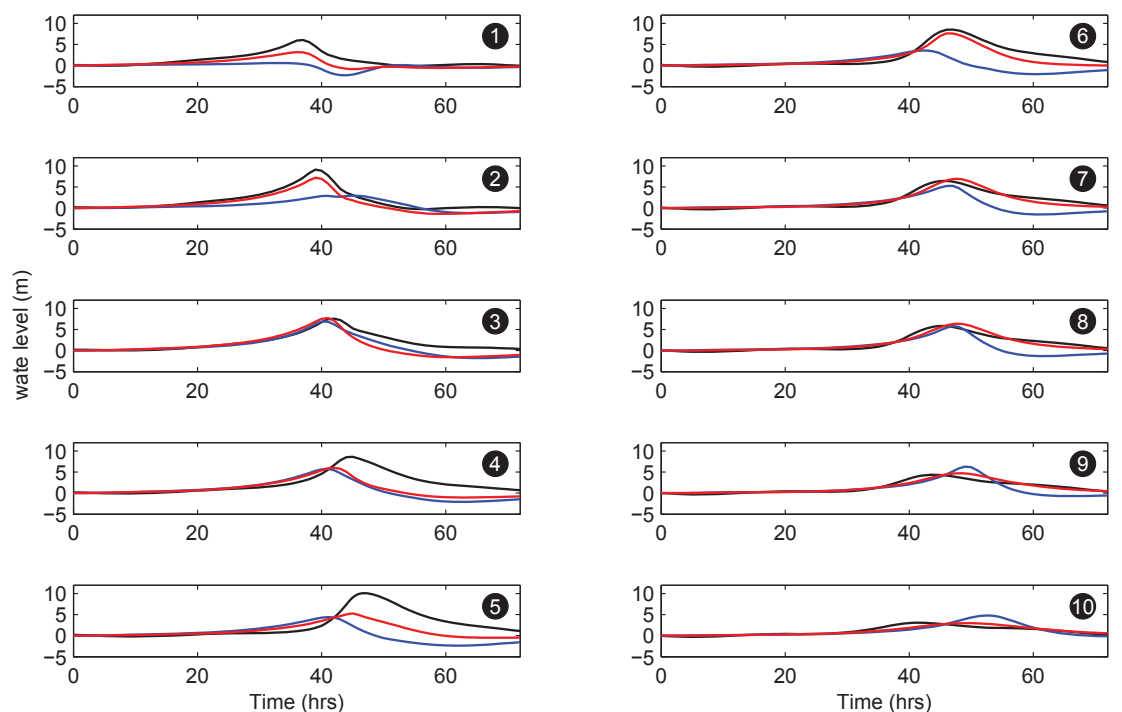

$-\mathrm{H}_{1}\left(\mathrm{SE}, \mathrm{P}_{2}\right)-\mathrm{H}_{2}\left(\mathrm{SW}, \mathrm{P}_{2}\right)-\mathrm{H}_{3}\left(\mathrm{~S}, \mathrm{P}_{2}\right)$

Figure 4.9: Surges at 10 locations are plotted as a function of time, induced by storms from southeastern direction $\left(H_{1}\right.$, black line), southwestern direction $\left(H_{2}\right.$, blue line) and southern direction $\left(H_{3}\right.$, red line). The locations are indicated by black circles with white numbers. Details in Tables 4.3 and 4.4 and Figure 4.6.

Table 4.5: Overview of peak surges ${ }^{\dagger}$ (in $\mathrm{m}$ ), as simulated at the selected locations $\left(P_{j}\right.$, for $\left.j=1,2, \cdots, 10\right)$ for the 13 storm scenarios listed in Table 4.4 .

\begin{tabular}{clcccccccccc}
\hline \multicolumn{2}{c}{ Scenario } & $P_{1}$ & $P_{2}$ & $P_{3}$ & $P_{4}$ & $P_{5}$ & $P_{6}$ & $P_{7}$ & $P_{8}$ & $P_{9}$ & $P_{10}$ \\
\hline$H_{1}$ & $\left(\mathrm{SE}, P_{2}\right)_{\text {ref }}$ & 6.0 & 9.1 & 7.5 & 8.6 & $\mathbf{1 0 . 1}$ & 8.5 & 6.4 & 5.8 & 4.4 & 3.1 \\
$H_{2}$ & $\left(\mathrm{SW}, P_{2}\right)$ & 0.6 & 2.9 & $\mathbf{6 . 8}$ & 5.7 & 4.4 & 3.6 & 5.3 & 5.8 & 6.3 & 4.8 \\
$H_{3}$ & $\left(\mathrm{~S}, P_{2}\right)$ & 3.2 & 7.2 & 7.6 & 6.0 & 5.1 & $\mathbf{7 . 7}$ & 6.9 & 6.4 & 4.7 & 3.0 \\
$H_{4}$ & $\left(\mathrm{SE}, P_{7}\right)$ & 2.8 & 3.5 & 2.0 & 1.0 & 0.7 & 2.1 & 4.8 & 5.5 & $\mathbf{6 . 7}$ & 5.6 \\
$H_{5}$ & (SE, $\left.P_{5}\right)$ & 4.5 & 6.6 & 4.0 & 2.5 & 5.8 & $\mathbf{8 . 4}$ & 7.8 & 7.3 & 5.5 & 3.8 \\
$H_{6}$ & (SE, $\left.P_{2}^{*}\right)$ & 5.6 & 8.4 & $\mathbf{9 . 7}$ & 9.6 & 9.4 & 6.9 & 5.0 & 4.5 & 3.3 & 2.4 \\
$H_{7}$ & (smaller) & 5.3 & 7.8 & 6.3 & 7.5 & $\mathbf{8 . 1}$ & 6.5 & 4.6 & 4.2 & 3.0 & 2.1 \\
$H_{8}$ & (larger) & 6.7 & 10.1 & 8.5 & 9.2 & $\mathbf{1 1 . 0}$ & 9.9 & 7.8 & 7.2 & 5.6 & 4.1 \\
$H_{9}$ & (slower) & 7.7 & $\mathbf{1 2 . 2}$ & 11.1 & 10.8 & 11.9 & 9.0 & 6.6 & 6.0 & 4.4 & 2.9 \\
$H_{10}$ & (faster) & 4.7 & 6.5 & 4.9 & 6.1 & 8.0 & $\mathbf{8 . 2}$ & 6.6 & 6.1 & 4.7 & 3.5 \\
$H_{11}$ & (weaker) & 4.6 & 6.9 & 5.7 & 6.6 & $\mathbf{7 . 7}$ & 6.5 & 4.9 & 4.4 & 3.3 & 2.3 \\
$H_{12}$ & (stronger) & 7.5 & 11.4 & 9.4 & 10.7 & $\mathbf{1 2 . 5}$ & 10.6 & 8.0 & 7.3 & 5.5 & 3.9 \\
\hline
\end{tabular}


landfall at location $P_{2}$ maximum winds are blowing offshore and onshore at location $P_{1}$ and $P_{3}$, respectively. Next, as the storm moves into the basin, the wind directions at $P_{3}$ turn to offshore. The storm makes second landfall at $P_{7}$, with winds blowing offshore at $P_{6}$ and onshore at $P_{7}$ and $P_{8}$. Finally, storm $H_{3}$ approaches the basin from the southern direction $\left(\phi=322.5^{\circ}\right)$. It also makes first landfall at location $P_{2}$, then moves into the basin and makes second landfall at $P_{5}$.

Figure 4.9 shows the resulting surge levels as a function of time at locations $P_{j}$ for $j=1,2,3, \cdots, 10$, where the black line describes the surge event for storm $H_{1}$, the blue line for storm $H_{2}$ and red line for storm $H_{3}$. Regarding the peak surge, storms from a southeastern direction are the most severe. Storm $H_{1}$ produces a peak of $10.1 \mathrm{~m}$ at $P_{5}$ (see Table 4.5 ), with maximum winds blowing onshore when making its second landfall. Storm $H_{1}$ moves over New Orleans coastal basin in a direction that produces the highest surge. It drives water from the Gulf of Mexico into the basin and traps the water until it makes second landfall. All stations exhibit a positive surge for this scenario. Alternatively, storm $H_{2}$ merely redistributes the water in the basin. This can be seen from the surge levels at $P_{1}$. No water set up occurs at this location and there is a negative surge due to the strong offshore winds when storm $\mathrm{H}_{2}$ moves over the Mississippi dike. A peak surge of $6.3 \mathrm{~m}$ is found at location $P_{9}$. For the New Orleans coastal basin, a storm from the southern direction is also severe. Storm $H_{3}$ first drives water to the left bottom corner of the basin $\left(P_{3}\right)$ and produces a surge level of $7.6 \mathrm{~m}$. The pile-up disappears afterwards, since the winds at location $P_{3}$ start blowing offshore as the storm moves on. Another peak surge of $7.7 \mathrm{~m}$ (Table 4.5 ) is observed at location $P_{6}$, where the maximum winds blow onshore. The sensitivity of the surge levels to the direction of storm strongly depends on location. For example, at $P_{3}$, a storm from a southern direction produces the highest surge (storm $H_{3}$ ), whereas at $P_{9}$, a storm from the southwestern direction produces the highest surge (storm $H_{2}$ ). We conclude that the most severe storms for the New Orleans coastal basin come from the southeastern direction.

\subsubsection{Sensitivity to landfall point}

In this section we investigate the influence of varying the point of landfall. Focusing on storms from a southeastern direction, we consider storms $H_{1}, H_{4}$, $H_{5}$ and $H_{6}$. The tracks of these storms are parallel to each other, with landfall points $P_{2}, P_{7}, P_{5}$ and $P_{2}^{*}$, respectively (see Table 4.4 and Fig. 4.6).

Similar to Fig.4.9, Fig.4.10 shows the surge levels at 10 locations forced by storms $H_{4}$ (chocolate line), $H_{5}$ (dark cyan line), $H_{1}$ (black line) and $H_{6}$ (grey thick line). As the chocolate line shows, $H_{4}$ induces a negative surge in the basin except at locations $P_{1}$ and $P_{10}$. This is because before landfall the winds first blow offshore at locations $P_{7}, P_{8}$ and $P_{9}$, leading to a set-down of water level at these locations. When making landfall, the winds blow onshore at $P_{7}$, $P_{8}, P_{9}$ and $P_{10}$, but still offshore at $P_{6}$. As the storm moves on, locations $P_{5}$, $P_{4}$ and $P_{3}$ also experience offshore winds. 

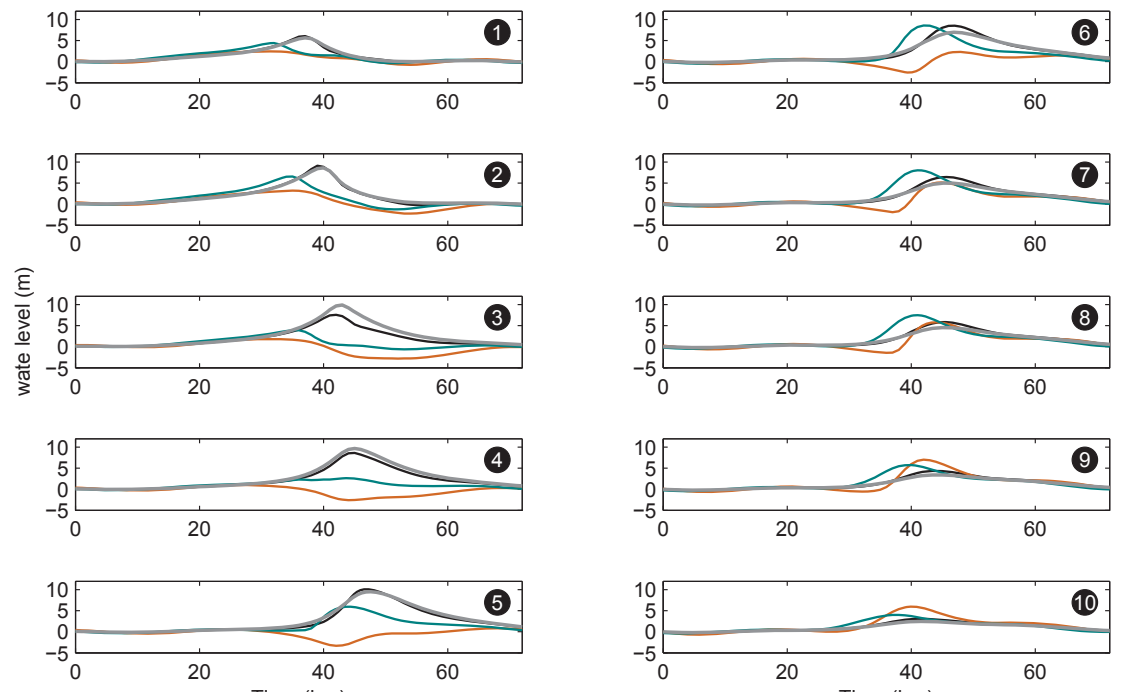

$$
-\mathrm{H}_{1}\left(\mathrm{SE}, \mathrm{P}_{2}\right)-\mathrm{H}_{4}\left(\mathrm{SE}, \mathrm{P}_{7}\right)-\mathrm{H}_{5}\left(\mathrm{SE}, \mathrm{P}_{5}\right)-\mathrm{H}_{6}\left(\mathrm{SE}, \mathrm{P}_{2}^{*}\right)
$$

Figure 4.10: Same as Fig 4.9, but now for storm scenarios with different points of landfall: $H_{1}$ (landfall at $\left.P_{2}\right), H_{4}\left(P_{7}\right), H_{5}\left(P_{5}\right)$ and $H_{6}\left(P_{2}^{*}\right)$. See Table 4.4 and Fig. 4.6. 

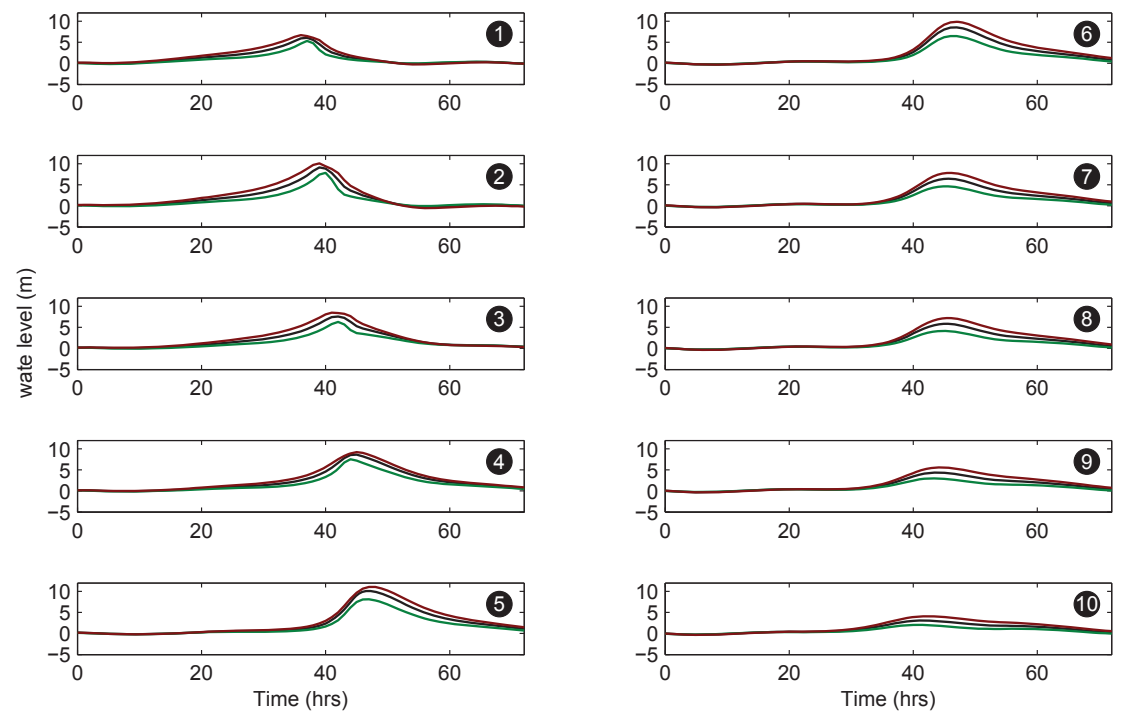

$$
-\mathrm{H}_{1}\left(\mathrm{SE}, \mathrm{P}_{2}\right)-\mathrm{H}_{7} \text { (smaller) }-\mathrm{H}_{8} \text { (bigger) }
$$

Figure 4.11: Same as Fig.4.9, but now for storm scenarios with different radii to maximum wind $R_{\max }$ : $50 \mathrm{~km}$ ( $H_{1}$, reference case), $30 \mathrm{~km}$ (smaller) and $70 \mathrm{~km}$ (larger). Also see detailed information of the storms can be found in Table 4.4.

The peak surge of $H_{5}$ is observed at location $P_{6}$, showing a water level of $8.4 \mathrm{~m}$ (see Table 4.5). This is to be expected, since the maximum winds blow to the coast at location $P_{6}$ when making landfall. Apart from $P_{6}$, we notice that there is another peak at $P_{2}$ with a height of $6.6 \mathrm{~m}$. The winds at this location blow onshore throughout the passing of the storm.

Storms $H_{1}$ and $H_{6}$ produce the highest surge. In particular, $H_{1}$ induces a peak of $9.8 \mathrm{~m}$ at $P_{5}$ (see Table 4.5 ). Surprisingly, $H_{6}$ also produces a peak of 9.7 $\mathrm{m}$ at $P_{3}$. Although the centre of storm $H_{6}$ does not move over the New Orleans coastal basin, the winds keep blowing to the left corner $\left(P_{3}, P_{4}\right.$ and $\left.P_{5}\right)$. Such effect is also observed in reality. In August 2012, Hurricane Isaac made landfall in southeastern Louisiana, following a similar track as $H_{6}$. Despite being only a category 1 hurricane, Isaac produced a surge of $3.4 \mathrm{~m}$ at Shell Beach, Louisiana (Fanelli and Wolcott, 2012), which is close to $P_{3}$.

\subsubsection{Sensitivity to storm size}

So far we considered a radius to maximum wind $\left(R_{\max }\right)$ of $50 \mathrm{~km}$. We now investigate what happens when varying the radius to maximum wind. To this end, we introduce scenarios $H_{7}$ and $H_{8}$, which are identical to $H_{1}$ with the exception of the radius to maximum wind being $30 \mathrm{~km}$ for $H_{7}$ and $70 \mathrm{~km}$ for $H_{8}$. This affects the area of water on which the winds act. The comparison of 

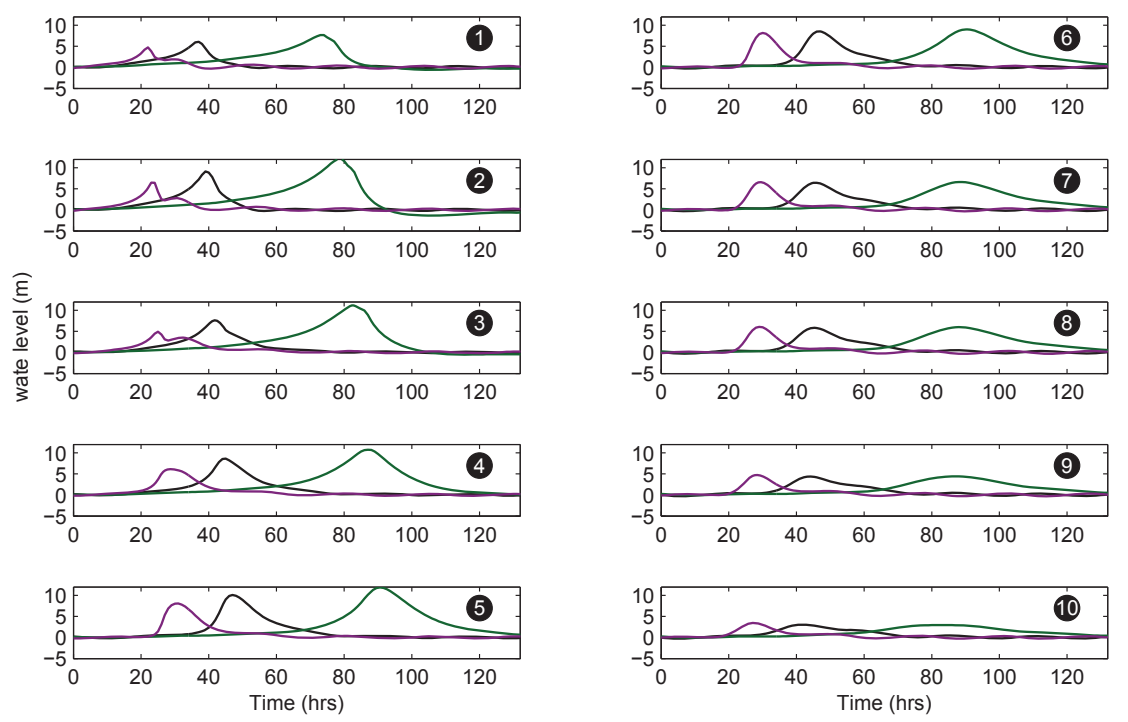

$$
-\mathrm{H}_{1}\left(\mathrm{SE}, \mathrm{P}_{2}\right)-\mathrm{H}_{9} \text { (slower) }-\mathrm{H}_{10} \text { (faster) }
$$

Figure 4.12: Same as Fig.4.9, but now for storm scenarios with different forward speed: $C_{\mathrm{fm}}=6 \mathrm{~m} \mathrm{~s}^{-1}\left(H_{1}\right.$, reference case), $3 \mathrm{~m} \mathrm{~s}^{-1}\left(H_{9}\right.$, slower $)$ and $10 \mathrm{~m} \mathrm{~s}^{-1}\left(H_{10}\right.$, faster). Detailed information of the storm scenarios can be found in Table 4.4.

surges induced by $H_{1}$ (black) $H_{7}$ (green) and $H_{8}$ (brown) is shown in Figure 4.11.

A storm of larger size influences a larger area. The effects are twofold. Firstly, more locations are influenced by a larger storm. For example, at location $P_{10}$, storm $H_{7}$ causes a minor effect, whereas storm $H_{8}$ causes a significant surge of $4.1 \mathrm{~m}$. Secondly, a larger storm leads to higher surge, particularly for the locations in the New Orleans coastal basin. Because the winds of storms coming from a southeastern direction blow into the basin when making landfall at $P_{1}$, a larger storm size thus drives more water into the basin. At location $P_{5}$, storm $H_{8}$ produces a surge of $11.0 \mathrm{~m}$. On average, increasing $R_{\max }$ from $30 \mathrm{~km}$ to 70 $\mathrm{km}$ leads to a $2.5 \mathrm{~m}$ increase of surge in the basin. The maximum effect appears at $P_{6}$, showing an increase of surge of $3.4 \mathrm{~m}$. Our results are consistent with those by Irish et al (2008). They found for a given intensity, surges vary by as much as $30 \%$ over a reasonable range of storm sizes.

\subsubsection{Sensitivity to forward speed}

Forward speed $\left(C_{\mathrm{fm}}\right)$ determines the time that the winds act on the water surface. So far, we used a speed of $6 \mathrm{~m} \mathrm{~s}^{-1}$. Now consider storm $H_{9}$ with $C_{\mathrm{fm}}=3$ $\mathrm{m} \mathrm{s}^{-1}$ and storm $H_{10}$ with $C_{\mathrm{fm}}=10 \mathrm{~m} \mathrm{~s}^{-1}$. The other parameters of these two storms are the same for storm $H_{1}$. Because of the different approach speeds, the landfall times are different, as marked in Fig 4.12 for the selected locations. 

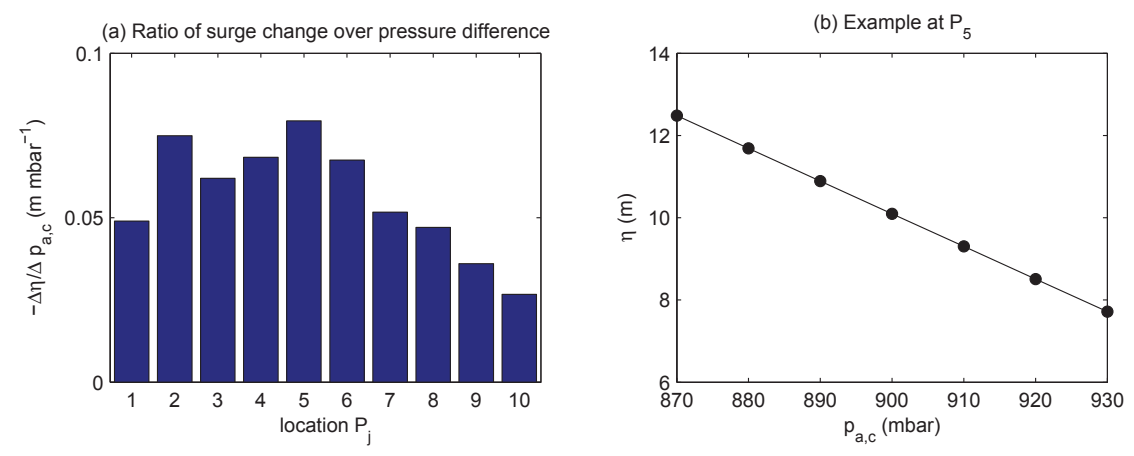

Figure 4.13: Dependency of simulated peak surge on central pressure $p_{a, c}:(a)$ ratio of surge change over pressure difference, $(b)$ example at $P_{5}$.

The results indicate that a storm with a slower forward speed produces higher surges. This is particularly true for the inner basin of the New Orleans coastal area, i.e. for locations $P_{2}, P_{3}, P_{4}, P_{5}$ and $P_{6}$. Storm $H_{9}$ produces the highest surge (dark green line). At location $P_{2}$ the dark green line shows a peak surge of $12.2 \mathrm{~m}$. Another surge peak is observed at $P_{5}$, showing a surge level of $11.6 \mathrm{~m}$. From $\S 4.5 .2$ and $\S 4.5 .3$, we know that a storm from the southeastern direction, which makes landfall at $P_{1}$, will drive water into the basin and trap the imported water. This effect is further intensified when the storm moves at a slower speed. However, this does not hold for locations away from the inner basin. For example, at locations $P_{9}$ and $P_{10}$, storm $H_{10}$ produces the highest surges. A storm with a higher forward speed will introduce higher wind speeds. Therefore, without the trapping effect of a semi-enclosed basin, such storms produce higher surges.

Another influence of forward speed is the duration time of a surge event. This can be seen from the last time of high water levels of the dark green line, the surge event last longer for storms with lower forward speeds.

\subsubsection{Sensitivity to central pressure}

To examine the influence of central pressure, we now linearly increase the central pressure from $p_{a, c}=870 \operatorname{mbar}\left(H_{12}\right)$ to $p_{a, c}=930 \operatorname{mbar}\left(H_{11}\right)$, with an interval of 10 mbar. other parameters of storms $H_{11}$ and $H_{12}$ are the same for $H_{1}$. Figure 4.13 shows the dependency of the simulated peak surge at 10 locations $P_{j}$ on central pressure. As expected, a storm of lower central pressure produces higher surges. The peak surge shows a linear dependence on central pressure (see right panel in Fig 4.13). However, this dependency varies over the locations. With the same decrease of central pressure, the increase of peak surges at locations $P_{2}, P_{4}$ and $P_{5}$ are more than at other locations. 


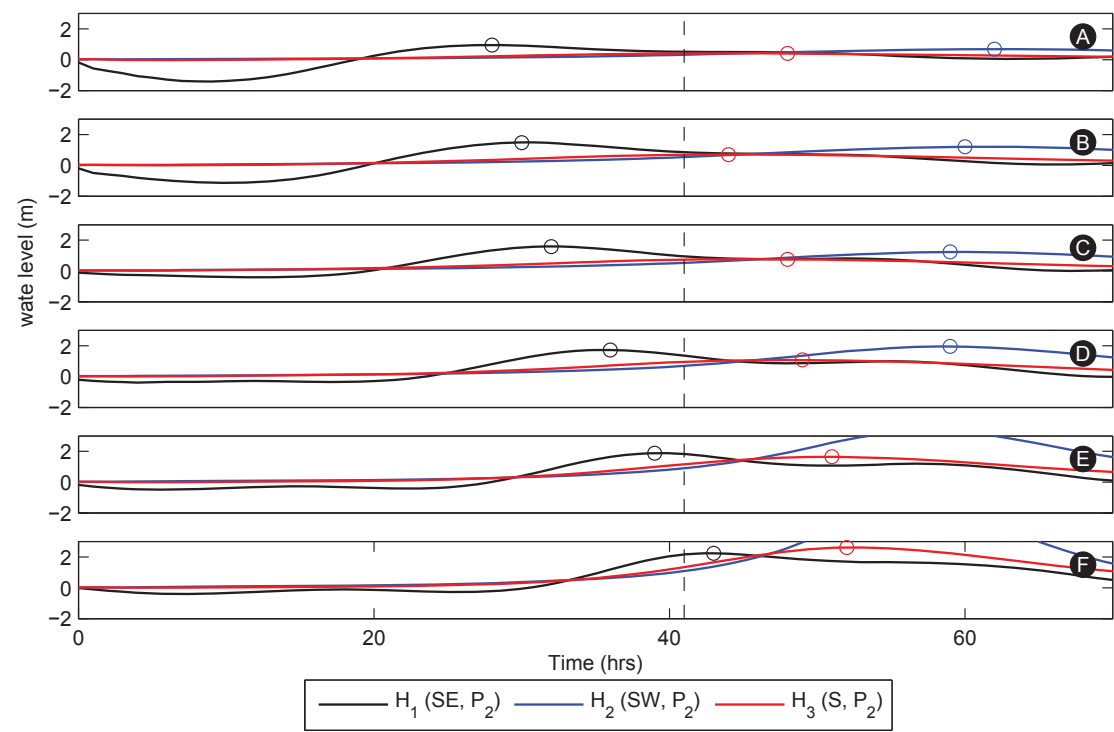

Figure 4.14: The water levels at locations from south point of Florida $\left(P_{A}\right)$ to New Orleans $\left(P_{F}\right)$ are plotted as a function of time, for storm $H_{1}$ (black line), storm $H_{2}$ (blue) and storm $H_{3}$ (red), with circles indicating the maximum surge. The black dashed vertical line denotes the landfall time of storm $H_{1}, H_{2}$ and $H_{3}$. The locations $P_{A}$ to $P_{F}$ are shown in Fig 4.6. 


\subsubsection{Forerunner}

Kennedy et al (2011) diagnose the forerunner surge of Hurricane Ike as being generated by Ekman set-up on the wide and shallow continental shelves along the western Louisiana and the northern Texas coasts. Such an Ekman set-up driven forerunner is most significant on the broad shallow continental shelf such as the northeastern shelf of the Gulf of Mexico. To investigate the influence of storm direction on the forerunner surge in our model results, we evaluate the water levels at locations from the south point of Florida $\left(P_{A}\right)$ to New Orleans $\left(P_{F}\right)$, for storms $H_{1}$ (black line), $H_{2}$ (blue) and $H_{3}$ (red); see Figure 4.14.

Only the surge induced by $H_{1}$ clearly display a set-up before landfall (denoted by the vertical black dashed line). The water level at location $P_{A}$ reaches to a peak of $0.9 \mathrm{~m} 13$ hours prior to landfall, as indicated by the black circle in Fig 4.14. The surge peak travels westward along the coast, i.e. from location $P_{A}$ to $P_{F}$. With a coastal bank on its right-hand side, the along-shore wind of storm $H_{1}$ generates an along-shelf current over a wide shelf, leading to an Ekman set-up due to the balance between the Coriolis force acting on the along-shelf current and the cross shelf gradient. This finding is consistent with Kennedy et al (2011) analysis of Hurricane Ike which moved over the Gulf of Mexico and attacked Galveston, Texas from the southeastern direction. For storms $\mathrm{H}_{2}$ and $H_{3}$, their tracks are too far away from the coast and their approaching direction are not in favor of generating Ekman set-up.

\subsubsection{Fourier spectrum of the elevation amplitude}

As shown in $§ 4.5 .2$, storms from the southeastern direction produce the highest surge. This is also seen from observed by the Fourier spectrum of the elevation amplitude. Figure 4.15 shows the absolute value of the elevation amplitude for the Fourier modes at locations $P_{3}, P_{5}$ and $P_{6}$ is shown for storm $H_{1}$ (Fig 4.15 a), $H_{2}$ (Fig $4.15 b$ ) and $H_{3}$ (Fig $4.15 c$ c). In general, the amplitude is lower for higher frequencies. The amplitudes of higher modes are negligible compared with the highest amplitude peak. This also supports our choice of Fourier modes $M$ and recurrence period $T_{\text {recur }}$.

For storm $H_{1}$, we notice that there is a local amplitude peak at mode 8 . The corresponding frequency $\omega_{8}=7.8 \times 10^{-5} \mathrm{~s}^{-1}$. This frequency is close to the quarter wave length frequency of New Orleans coastal basin $\omega_{\text {quarter }}=$ $7.7 \times 10^{-5} \mathrm{~s}^{-1}$, where $\omega_{\text {quarter }}=\frac{2 \pi \sqrt{g h}}{4 L}$ with basin length $L \approx 110 \mathrm{~km}$ is the frequency for which the frictionless shallow water wavelength equals 4 times of the basin length. This finding is consistent with the resonant mechanism studied in chapter 3, where we observe the resonance frequencies associated with (odd multiples of) the quarter wavelength for a topographic step with a shallow coastal part. As can be seen from Figure 4.5, the topography of New Orleans indeed displays a quick drop at the mouth of the basin, thus forming a shallow coastal part.

Instead of lowest frequency, the amplitude surge peak for storm $\mathrm{H}_{2}$ appears at mode 5. This is because the travelling time through the domain of storm $\mathrm{H}_{2}$ is smaller than $H_{1}$. Therefore, the dominant forcing frequency of $H_{2}$ is higher 

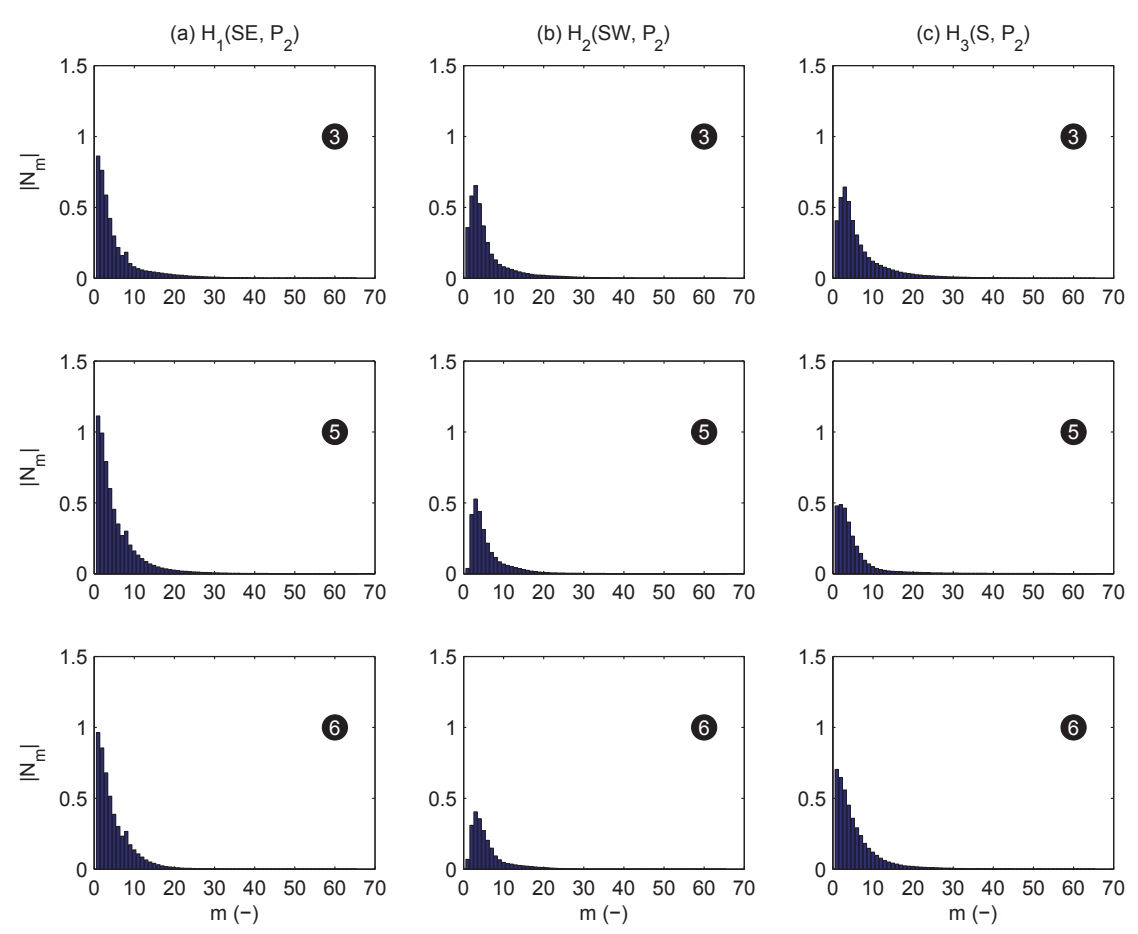

Figure 4.15: The elevation amplitude of Fourier modes at location $P_{3}, P_{5}$ and $P_{6}$ for three storm scenarios: (a) $H_{1},(b) H_{2}$ and (c) $H_{3}$. 
than $H_{1}$.

\subsection{Discussion and conclusion}

In this study, we presented a new method to study storm surges. An idealised process-based model has been developed to simulate surge levels in coastal basins. Focusing on the New Orleans coastal basin, the geometry and bathymetry are schematised. The storm forcings, wind and pressure field, are represented using the Holland-B model. Through Fourier expansion these forcings are converted into periodic signals at different frequencies. Using the Finite Element Method, the solutions at each frequency are obtained. Note that the vertical profile of the flow field are fully analytical. The surge levels are then the superposition of solutions at different frequencies.

To analyze the model results, water levels at 10 locations have been evaluated. The model is first applied to simulate the Hurricane Katrina surge in the New Orleans coastal basin. Our model qualitatively captures the Katrina surge in the New Orleans coastal basin and also produces reasonable quantitative results.

Next, the influence of storm parameters has been investigated by comparing the surge levels in the New Orleans coastal basin for storms with different parameters. Model results show that for the New Orleans coastal basin, storms from southern and southeastern directions produce the highest surge. Storms from these directions drive water into the basin and trap the imported water until making landfall. In particular, a southeastern storm causes an overall set-up in the basin. Alternatively, storms from southwestern direction only cause local high water and redistribute the water inside the basin. From our analysis, the track of the most severe storm for the New Orleans coastal area is a southeastern storm making landfall at the head of the Mississippi dike. This combination produces the highest surge of $9.8 \mathrm{~m}$. Due to the spatial variation of surge levels, the sensitivity of the surge level at a given location strongly depends on location. Only storms from the southeastern direction cause a forerunner surge along the coast from south Florida to New Orleans.

The surges in the basin are also sensitive to the radius to maximum wind and forward speed. The former parameter influences the area of water on which winds act whereas the latter parameter determines the time over which the winds act. A larger storm with slower forward speed following the most dangerous track produces highest surge in the basin. The central pressure also influences the surges, but to a lesser extent than the other parameters.

Hurricane Katrina approached the New Orleans coastal area from the southeastern direction, and made landfall at location $P_{2}$. The combination of this track and geometry leads to high surge along the north bank of the New Orleans coastal basin (location $P_{5}, P_{6}, P_{7}$ and $P_{8}$ ). On the other hand, of the left corner of the New Orleans coastal basin (location $P_{3}$ ), a storm from southeastern direction running below and parallel to the Mississippi dike such as Hurricane Issac produces the highest surge, since all winds blow into the basin when Hurricane Issac approaches and maximum wind blows to the corner when it passes. 
Acknowledgement: This work extends the MSc-work by L. Straatsma, supervised by W.L. Chen, P.C. Roos, H.M. Schuttelaars, M. van Ledden, J.P.M. Mulder and S.J.M.H. Hulscher. Dr. Dietrich is gratefully acknowledged for providing the data from the ADCIRC simulations. This work is partly funded by the Chinese Scholarship Council and partly by the research programme 'Impact of climate change and human intervention on hydrodynamics and environmental conditions in the Ems-Dollart estuary: an integrated data-modelling approach'. The latter project is financed by the Bundesministerium fur Bildung und Forschung (BMBF) and by the Netherlands Organization for Scientific Research (NWO), as part of the international Wadden Sea programme (GEORISK project). 


\section{Appendix}

\section{A Details of the derivation}

\section{A.1 Vertical profiles from horizontal momentum equations}

Here we present the details of the vertical structure of the flow at frequency $\omega_{m}$. First we define rotating flow components according to $q_{m}^{ \pm}=u_{m} \pm \mathrm{i} v_{m}$ with complex amplitudes $Q_{m}^{ \pm}$, such that $U_{m}=\left(Q_{m}^{+}+Q_{m}^{-}\right) / 2$ and $V_{m}=\left(Q_{m}^{+}-Q_{m}^{-}\right) /(2 \mathrm{i})$. The rotating flow solution contains three contributions, proportional to the surface gradient the wind stress and the pressure gradient, respectively:

$$
Q_{m}^{ \pm}(z)=Q_{m, \eta}^{ \pm}(z) \mathcal{L}^{ \pm} N_{m}+Q_{m, p}^{ \pm} \mathcal{L}^{ \pm} P_{\mathrm{a}, \mathrm{m}}+Q_{m, \mathrm{w}}^{ \pm}(z) T_{m}^{ \pm}
$$

with complex operators $\mathcal{L}^{ \pm}=\partial / \partial x \pm \mathrm{i} \partial / \partial y$ and rotating wind forcing amplitudes $T_{m}^{ \pm}=T_{m}^{(x)} \pm \mathrm{i} T_{m}^{(y)}$ (wind stress divided by density). The vertical structures read

$$
\begin{aligned}
Q_{m, \eta}^{ \pm}(z) & =\frac{g\left[\cosh \lambda_{m}^{+} z-\alpha_{\mathrm{c}, m}^{ \pm}\right]}{\alpha_{\mathrm{c}, m}^{ \pm} K \lambda_{m}^{ \pm 2}}, \\
Q_{m, p}^{ \pm}(z) & =\frac{\left[\cosh \lambda_{m}^{+} z-\alpha_{\mathrm{c}, m}^{ \pm}\right]}{\rho \alpha_{\mathrm{c}, m}^{ \pm} K \lambda_{m}^{ \pm 2}} \\
Q_{m, \mathrm{w}}^{ \pm}(z) & =\frac{\alpha_{\mathrm{c}, m}^{ \pm} \sinh \lambda^{ \pm} z+\alpha_{\mathrm{s}, m}^{ \pm} \cosh \lambda_{m}^{ \pm} z}{\alpha_{\mathrm{c}, m}^{ \pm} K \lambda_{m}^{ \pm}}
\end{aligned}
$$

with $\lambda_{m}^{ \pm 2}=-\mathrm{i}\left(\omega_{m} \mp f\right) / K$ and $\alpha_{\mathrm{c}, m}^{ \pm}=\cosh \lambda_{m}^{ \pm} h+s^{-1} K \lambda_{m}^{ \pm} \sinh \lambda_{m}^{ \pm} h$ and $\alpha_{\mathrm{s}, m}^{ \pm}=$ $\sinh \lambda_{m}^{ \pm} h+s^{-1} K \lambda_{m}^{ \pm} \cosh \lambda_{m}^{ \pm} h$. The vertical integral is given by

$$
\left\langle Q_{m}^{ \pm}\right\rangle=\left\langle Q_{m, \eta}^{ \pm}\right\rangle \mathcal{L}^{ \pm} N_{m}+\left\langle Q_{m, p}^{ \pm}\right\rangle \mathcal{L}^{ \pm} P_{\mathrm{a}, m}+\left\langle Q_{\mathrm{w}, m}^{ \pm}\right\rangle T_{m}^{ \pm}
$$

with

$$
\begin{aligned}
\left\langle Q_{m, \eta}^{ \pm}\right\rangle & =\frac{g\left[\sinh \lambda_{m}^{ \pm} h-\alpha_{\mathrm{c}, m}^{ \pm} \lambda_{m}^{ \pm} h\right]}{\alpha_{\mathrm{c}, m}^{ \pm} K \lambda_{m}^{ \pm 3}}, \\
\left\langle Q_{m, p}^{ \pm}\right\rangle & =\frac{\left[\sinh \lambda_{m}^{ \pm} h-\alpha_{\mathrm{c}, m}^{ \pm} \lambda_{m}^{ \pm} h\right]}{\rho \alpha_{\mathrm{c}, m}^{ \pm} K \lambda_{m}^{ \pm 3}}, \\
\left\langle Q_{m, \mathrm{w}}^{ \pm}\right\rangle & =\frac{\alpha_{\mathrm{c}, m}^{ \pm}\left[1-\cosh \lambda_{m}^{ \pm} h\right]+\alpha_{\mathrm{s}, m}^{ \pm} \sinh \lambda_{m}^{ \pm} h}{\alpha_{\mathrm{c}, m}^{ \pm} K \lambda_{m}^{ \pm 2}}
\end{aligned}
$$

The two cases $\omega_{m}= \pm f$ require alternative expressions for either $Q_{m}^{+}$or $Q_{m}^{-}$. If $\omega_{m}=+f$ we must replace the $Q_{m}^{+}$-expressions in Eqs.(4.21)-(4.24); if $\omega_{m}=-f$ we must replace the $Q_{m}^{-}$-expressions. They must be replaced with

$$
\begin{aligned}
& Q_{m, \eta}^{ \pm}(z)=\frac{g h^{2}}{K}\left[\frac{1}{2}\left(\frac{z}{h}\right)^{2}-\frac{1}{2}-\frac{K}{s h}\right] \\
& Q_{m, p}^{ \pm}(z)=\frac{h^{2}}{\rho K}\left[\frac{1}{2}\left(\frac{z}{h}\right)^{2}-\frac{1}{2}-\frac{K}{s h}\right] \\
& Q_{m, \mathrm{w}}^{ \pm}(z)=\left[1+\left(\frac{z}{h}\right)+\frac{K}{s h}\right]
\end{aligned}
$$


and

$$
\begin{aligned}
\left\langle Q_{m, \eta}^{ \pm}\right\rangle & =-\frac{g h^{3}}{K}\left[\frac{1}{3}+\frac{K}{s h}\right], \\
\left\langle Q_{m, p}^{ \pm}\right\rangle & =-\frac{h^{3}}{\rho K}\left[\frac{1}{3}+\frac{K}{s h}\right], \\
\left\langle Q_{m, \mathrm{w}}^{ \pm}\right\rangle & =\frac{h^{2}}{K}\left[\frac{1}{2}+\frac{K}{s h}\right] .
\end{aligned}
$$

In the above expressions, the no-slip condition corresponds to taking $s^{-1}=0$. Alternatively, the free-slip limit is obtained when taking the limit $s \downarrow 0$.

\section{A.2 Elliptical problem for $N$}

At frequency $\omega_{m}$, depth-integration of the continuity equation (4.3), with the aid of boundary conditions Eq. (4.4) gives, in terms of the complex amplitudes of surface elevation and the rotating velocity components.

$$
-\mathrm{i} \omega_{m} N_{m}+\frac{\partial}{\partial x} \underbrace{\left(\frac{\left\langle Q_{m}^{+}\right\rangle+\left\langle Q_{m}^{-}\right\rangle}{2}\right)}_{\left\langle U_{m}\right\rangle}+\frac{\partial}{\partial y} \underbrace{\left(\frac{\left\langle Q_{m}^{+}\right\rangle-\left\langle Q_{m}^{-}\right\rangle}{2 \mathrm{i}}\right)}_{\left\langle V_{m}\right\rangle}=0,
$$

Substitution of Eq.(4.24) gives the elliptical equation for $N_{m}$ which is identical to Eq.(4.14) in the main text:

$$
\boldsymbol{\nabla}_{\mathrm{h}} \cdot\left[\left\langle\mathbf{D}_{m}\right\rangle \nabla_{\mathrm{h}} N_{m}\right]-\mathrm{i} \omega_{m} N_{m}=-\nabla_{\mathrm{h}} \cdot\left\langle\mathbf{r}_{m}\right\rangle,
$$

with

$$
\mathbf{D}_{m}=\left[\begin{array}{cc}
C_{m}^{+} & C_{m}^{-} \\
-C_{m}^{-} & C_{m}^{+}
\end{array}\right], \quad \mathbf{r}_{m}=\left[\begin{array}{c}
R_{m}^{+} \\
R_{m}^{-}
\end{array}\right] .
$$

The $C_{m}^{+}$and $C_{m}^{-}$coefficients are given by

$$
C_{m}^{+}=\frac{1}{2}\left[Q_{m, \eta}^{+}+Q_{m, \eta}^{-}\right], \quad C_{m}^{-}=\frac{1}{2 \mathrm{i}}\left[Q_{m, \eta}^{+}-Q_{m, \eta}^{-}\right]
$$

The boundary conditions presented in Eqs.(4.16) of the main text follow from depthintegration of the momentum equations (4.1)-(4.2). The coefficients $R_{m}^{+}$and $R_{m}^{-}$are given by

$$
\begin{aligned}
& R_{m}^{+}=\frac{1}{2}\left[Q_{m, \mathrm{w}}^{+} T_{m}^{+}+Q_{m, \mathrm{w}}^{-} T_{m}^{-}\right]+\frac{1}{2}\left[Q_{m, p}^{+} \mathcal{L}^{+} P_{\mathrm{a}, \mathrm{m}}+Q_{m, p}^{-} \mathcal{L}^{-} P_{\mathrm{a}, \mathrm{m}}\right] \\
& R_{m}^{-}=\frac{1}{2 \mathrm{i}}\left[Q_{m, \mathrm{w}}^{+} T_{m}^{+}-Q_{m, \mathrm{w}}^{-} T_{m}^{-}\right]+\frac{1}{2 \mathrm{i}}\left[Q_{m, p}^{+} \mathcal{L}^{+} P_{\mathrm{a}, \mathrm{m}}-Q_{m, p}^{-} \mathcal{L}^{-} P_{\mathrm{a}, \mathrm{m}}\right]
\end{aligned}
$$

\section{A.3 Vertical flow velocity}

The vertical flow velocity amplitudes at any depth $z$ are given by

$$
W_{m}(z)=-\left\lfloor C_{m}^{+}\right\rfloor\left(\frac{\partial^{2} N_{m}}{\partial x^{2}}+\frac{\partial^{2} N_{m}}{\partial y^{2}}\right)-\left\langle C_{m}^{+}\right\rangle\left(\left\lfloor\frac{\partial R_{m}^{+}}{\partial x}\right\rfloor+\left\lfloor\frac{\partial R_{m}^{+}}{\partial y}\right\rfloor\right),
$$

where floor brackets indicate integration from bottom to $z$, i.e. $\lfloor\cdot\rfloor=\int_{-h}^{z} \cdot \mathrm{d} z$. This expression can be simplified further by using the differential equation (4.14) for $N_{m}$ to eliminate the Laplacian of $N_{m}$. 


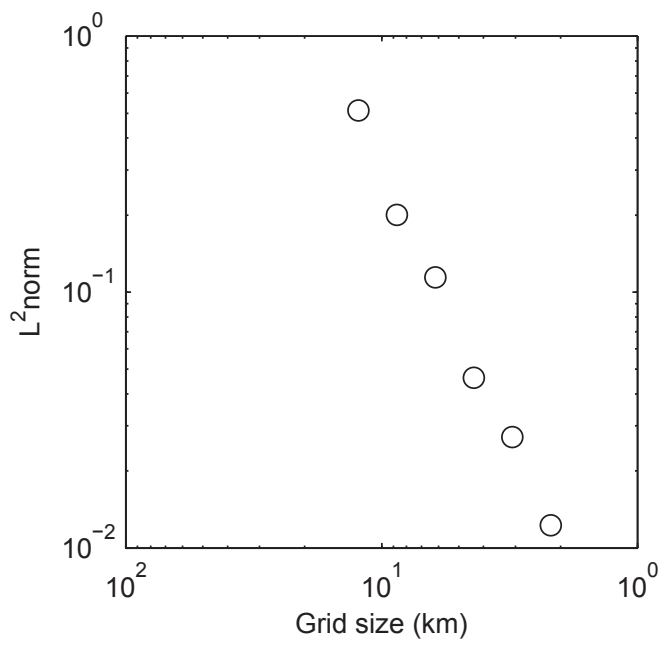

Figure 4.16: $L^{2}$-norm of the solution for different values of the grid size within the local basin of New Orleans.

\section{B Convergence test for Hurricane Katrina surge}

The storm surge model uses the Finite Element Method which divides the whole domain into discretised triangular mesh and calculate solution at these meshes. In principle, the numerical solution should converge to an exact solution when the size of the triangular mesh goes to zero (which means the number of nodes in the domain goes to infinity). A model with a fine grid gives a more accurate result. However, a calculation with a fine grid takes more time than with a coarse grid. Therefore, an appropriate grid size for the purpose of the sensitivity study should give an accurate result while taking acceptable time. To find a proper grid size for this study, we did a convergence test for the local coastal basin of New Orleans. Several simulation runs are made with different grid sizes. We calculate the $L^{2}$-norm, $\left\|E_{\bar{h}}\right\|_{2}$

$$
\left\|E_{\bar{h}}\right\|_{2}=\left(\iint_{\Omega}\left|N_{\bar{h}}-N_{\bar{h}}^{*}\right|^{2}\right)^{1 / 2},
$$

where $N_{\bar{h}}$ is the numerical solution for grid size $\bar{h}$, and $N_{\bar{h}}^{*}$ the numerical solution for finest grid $\left(N_{\bar{h}}^{*}=1 \mathrm{~km}\right)$. Results are presented in Figure 4.16. The Figure displays an order one convergence. For the New Orleans coastal area and boundaries, a grid of size of $4 \mathrm{~km}(\bar{h}=4 \mathrm{~km})$ is used. For the wider part of the domain a bigger grid size is used $(\bar{h}=8 \mathrm{~km})$. This results in 35748 grid points (see Figure 4.5) and 9 hours of calculation on a regular pc for the Hurricane Katrina surge simulation.

\section{C Fourier representation of atmospheric forcing}

The Fourier transformation applied in the model decomposes atmospheric forcings into periodic signals at different frequencies. Analogous to the number of elements, increasing the number of modes $(M)$ gives a more accurate representation. However, 

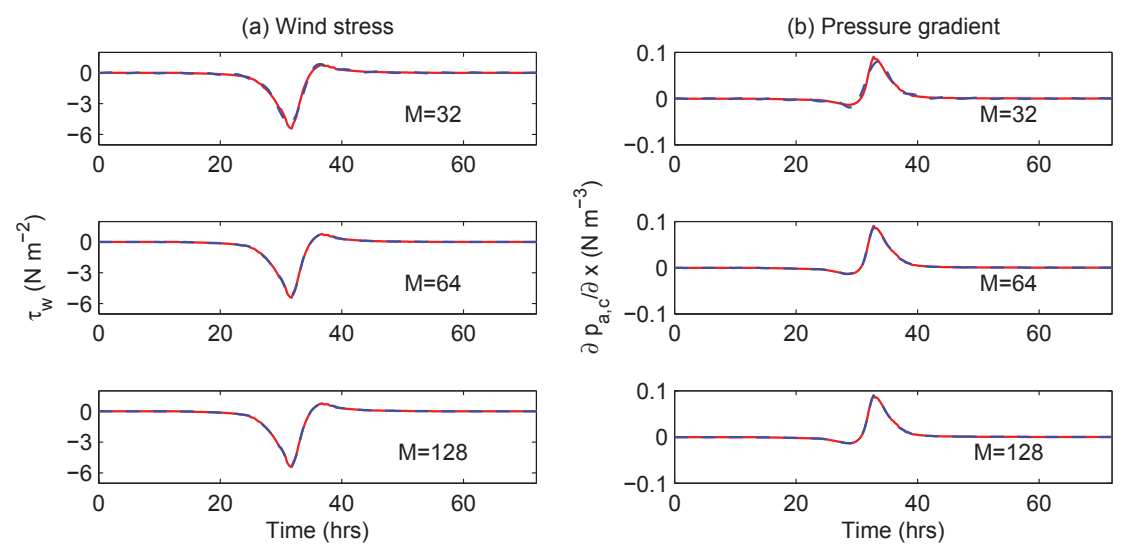

- real time forcings - - - Fourier representative

Figure 4.17: Real and Fourier representative atmospheric forcings of Hurricane Katrina at $P_{1}$, showing $(a)$ wind stress, $(b)$ pressure gradient, with 32, 64 and 128 modes. The recurrence period $T_{\text {recur }}$ is 7 days.

calculation with large $M$ consumes much more time. The number of modes is also related to the recurrence period of the storm, the longer the period the more modes are needed for the same accuracy.

Figure 4.17 shows the Fourier representative of the atmospheric forcings for different $M$ modes. As is illustrated, a combination of $T_{\text {recur }}=7$ days and $M=64$ gives a good representation of Hurricane Katrina forcings, and the calculation time is acceptable for the purpose of a sensitivity study. 


\section{Chapter 5}

\section{Discussion, conclusions and recommendations}

In this thesis the influence of geometry, topography and storm characteristics on storm surges in coastal basins is described. In this final chapter, we first discuss the main characteristics of the new model as well as the added value of the present study ( $\$ 5.1)$. We then present the main conclusions, answering the research questions $(\S 5.2)$, and addressing the general aim of this thesis (§5.3). Finally, $\S 5.4$ contains recommendations for further research.

\subsection{Discussion}

The model developed in this study has several important characteristics. Firstly, the model solves the linearised shallow water equations. This allows us to isolate the contributions of various mechanisms and to calculate the surge response as superposition of responses at different frequencies. The exclusion of nonlinear effects means that the advection and the tide-surge interaction are neglected, which may explain our underestimation of the simulated surge levels for the Katrina surge.

Regarding the open boundary condition, in chapter 3, we imposed a non-reflective boundary condition. With this boundary condition, the wave generated inside the basin can freely propagate away, thus causing no reflection at the boundary. The outer region is represented as an infinitely long channel with uniform depth. In chapter 4, we imposed a Dirichlet boundary condition, where the water levels at the open boundary are calculated by the inverted barometer effect.

In this study, we have applied this model to the New Orleans coastal basin, but this is not a restriction. Due to the FEM-approach we use, the model can deal with complex large-scale geometries and complex large-scale topographies. Regarding meteorological forcings, the Holland-B model used in this study can simulate tropical storms. To simulate extratropical storms, it should be replaced by a more suitable model such as the Norwegian cyclone model (Schultz and Vaughan, 2011).

Compared with the existing numerical models that give discretised solutions both in time and space, the model developed in this study is more efficient regarding calculation time. This is because of the model's linearity, enabling a spectral approach. Furthermore, the vertical calculations (analytical) is decoupled from the horizontal calculations (FEM). A completely analytical vertical profile of the flow field is important in capturing Ekman dynamics which contributes to the Ekman driven set-up forerunner. Due to the focus on surge level, not much attention has been paid to the velocity field, but the methodology applied in this thesis allows us to express the velocity in terms of surface elevation and forcings. This feature will facilitate future studies on the 
transport of sediments, nutrients and pollutants. Furthermore, this model calculates response as a superposition of solutions at different frequencies, allowing us to link the response of a coastal basin to forcings with its resonant properties.

Compared with the simple analytical models that are restricted to basins with simple geometry and topography, this model is flexible regarding geometries and topographics. Further, it includes more physics, combining three-dimensional flow, including Coriolis effect, bottom friction and transient response. Hence, our idealised process-based model bridges the gap between the theoretical model studies (not sitespecific) and the computationally expensive detailed site-specific numerical simulation models.

\subsection{Answers to the research questions}

Q1. How does the frequency response of a closed rotating basin depend on basin dimensions, the spatial structure of the wind forcing and bottom friction?

The results of Chapter 2 show that the frequency response of a closed rotating basin of uniform depth, focusing on the surface elevation at an evaluation point midway one of the cross-basin boundaries, is sensitive to the spatial structure of the wind forcing. Without rotation, a spatially uniform wind produces the classical resonance peaks at $L / \lambda=\frac{1}{2}, \frac{3}{2}, \cdots$, where $L$ is the basin length and $\lambda$ the shallow water wave length. Alternatively, divergent wind gives peaks at $L / \lambda=1,2, \cdots$. Including the Coriolis effect causes these peaks to shift.

If the cross-wind basin dimension $B$ is not small compared to the basin length $L$, cross-wind dynamics produces peaks at frequencies significantly lower than obtained by Ponte (2010). These cross-wind dynamics can be triggered by several mechanisms. Firstly, a wind forcing with nonzero curl produces cross-wind variations in elevation and thus cross-basin flow and oscillations that may be resonant. Secondly, the Coriolis acceleration of the along-wind flow also produces cross-basin oscillations. As discussed above, this along-wind pattern depends on the spatial pattern of the wind forcing, so these rotation-induced peaks will be different for spatially uniform wind, divergent wind and curl wind.

In each of the above cases, the main effect of increasing friction is a lowering of the peaks.

Q2. What is the influence of basin-scale topography on the frequency response of largescale coastal basins subject to wind forcing?

In Chapter 3 it was shown that topographic elements cause the resonance peaks to shift in the frequency domain through their effect on local wave speed. The results are obtained using an idealised process-based model and evaluated in terms of the elevation amplitude averaged over the basin's landward end. Coastal basins are represented by a semi-enclosed rectangular inner region, forced by wind. It is connected to an outer region (represented as an infinitely long channel) without wind forcing, which allows waves to freely propagate outward. The wind field is spatially uniform in the core of the inner region and has an arbitrary angle with respect to the along-basin direction.

Without the Coriolis effect, the response of a basin with uniform depth to alongbasin wind shows maxima at $L / \lambda=\frac{1}{2}, \frac{3}{2}, \cdots$. Because waves are allowed to propagate away into the outer sea, these maxima are much weaker than the peaks in the frequency response to cross-basin wind. For a basin with a crossbasin topographic step and a coastal part with a shallow water depth, the res- 
onance frequencies occur at $L_{\mathrm{c}} / \lambda_{\mathrm{c}}=\frac{1}{4}, \frac{3}{4}, \cdots$, where $L_{\mathrm{c}}$ is the length of coastal part and $\lambda_{c}$ being the shallow water wave length on the coastal part. These frequencies increase when increasing the depth of the coastal part. Conversely, when the depth of the coastal part is sufficiently deep, the coastal part displays a spatially uniform elevation pattern ('pumping mode'). Smoothening the topographic steps with sinusoidally shaped slopes shows that increasing the slope length shifts the maximum to slightly higher frequencies. The response to a linear along-basin bed profile in the inner region (along-basin wind only) shows higher response for increasing slopes (while fixing the depth in the outer region), because of the reduced depth in the coastal part. Varying the central depth of a parabolic cross-basin profile (along-basin wind only), while keeping the averaged depth the same, only weakly modifies the frequency response.

The Coriolis effect causes (strong) resonance peaks associated with cross-basin modes (which without rotation only appear in response to cross-basin wind) to emerge also in response to along-basin wind. It also introduces peaks at the inertial frequency.

Q3. What is the influence of storm characteristics on surge response in the New Orleans coastal basin?

In Chapter 4 we studied the response of the New Orleans coastal basin to storms with different characteristics. The model is first used in a hindcast study of the surges in the New Orleans coastal basin induced by Hurricane Katrina. Our model qualitatively captures the Katrina surge in the New Orleans coastal basin and also produces reasonable quantitative results. This gives confidence in the model, allowing for a sensitivity study of storm surges to storm characteristics. To investigate the influence of storm characteristics, the surges induced by storms of varying characteristics, i.e. storm size, central pressure, direction, landfall point and forward speed, are calculated. The results are evaluated in terms of water levels at various locations along the New Orleans coast.

Results show that the direction and landfall point of the storm are the most important parameters. For the New Orleans coastal basin, storms from southern and southeastern directions produce the highest surge. Storms from these directions drive water into the basin and trap the imported water until it makes landfall. In particular, a southeastern storm causes an overall set-up in the basin. The track of the most severe storm for the New Orleans coastal basin is a southeastern storm making landfall at the seaward end of the Mississippi dike. This combination produces the highest surge level. Due to the spatial variation of surge levels, the sensitivity of the surge level at a given location strongly depends on location. Only storms from the southeastern direction cause a forerunner surge.

The surges in the basin are also sensitive to the radius to maximum wind and the forward speed. The former parameter influences the horizontal area of water forced by windstress, whereas the latter parameter determines the time span during which wind forcing takes place. A larger storm with slower forward speed produces even higher surge levels. The central pressure also influences the surges, but to a lesser extent than the other parameters. 


\subsection{Overall conclusions}

The goal of the research is to understand storm surge processes in large-scale coastal basins, particularly the influence of geometry, topography and storm characteristics on set-up at the coast.

In this thesis, we have developed an idealised process-based model to study the storm surge process in large-scale coastal basins. These processes are linked to basin's resonance properties, which are found to depend on the spatial pattern of the wind forcing, the along-wind basin dimensions, as well as the influence of rotation. Moreover, both geometry and topography strongly influence the storm surges, as they alter the resonance properties of coastal basin.

The model has been applied to simulate the surge of Hurricane Katrina, demonstrating that the model is able to qualitatively simulate surge levels. A sensitivity study of surges in the New Orleans coastal basin to storm characteristics showed that the direction and landfall point of the storm are the most important storm parameters in determining the surge levels. They are shown to affect maximum surge, timing of the surge, spatial distribution of the surge levels along the coast as well as the duration time of high water events.

From a broader perspective, the results obtained in this thesis help the protection of coastal areas and the design of coastal zone management strategies. In particular, the idealised process-based model can serve as a quick estimation tool for the water levels and possible inundations induced by storms. Due to its flexibility regarding geometry and topography, the model can be easily applied to other locations. Due to the short calculation time, it is suitable for extensive sensitivity studies. These properties position the new idealised process-based model in between the theoretical model studies (not site-specific) and the computationally expensive detailed numerical simulation models.

\subsection{Recommendations}

Even though this thesis contributes to the fundamental knowledge of the storm surge processes in large scale coastal basins, various questions remain. To direct future research and to further improve the model, we formulate the following recommendations.

- In this thesis, we have neglected nonlinear effects. This facilitates our understanding of storm surge processes, allowing us to unravel different mechanisms. On the other hand, it limits the applicability of this model. Nonlinear effects such as tide-surge interaction can be significant, especially in coastal basins with complex topography. The influence of nonlinear effects on the frequency response of coastal basins, and storm surge at the coast is not clear.

- One obvious step is to systematically study the influence of complex coastlines, both on the frequency response and the storm surge. A basin of complex coastlines may elongate the travelling distance of waves, and also introduce dynamics in multiple directions. Moreover, depending on the shape, the wave can be trapped and possibly generate local resonance. This is also important for storm surges. With more complex coastlines, we can better understand high water events in reality.

- Another step would be to study the combined effect of storm characteristics. In this thesis, the influence of storm characteristics is studied for each storm 
characteristic separately. However, an extra-high water event is usually triggered by the combined effect of several factors. For instance, the sensitivity of storm surge to central pressure and storm size is higher with a specific angle of landfall. Future work should consider more scenarios that investigate the effects of different combinations of storm parameters.

- The storm surge model can be applied to investigate the influence of human intervention on storm surges in coastal basins, and thus contributes to the design of large-scale sand extractions and land reclamations. By altering the configuration of the sea floor and basin shape, sand extraction and land reclamation may modify the local hydrodynamics. How these changes affect set-up due to wind and storm is unknown.

- A possible step is to study the morphodynamic effect of a storm. Storms not only induce high surge levels along the coast, but also give rise to sediment transport which may reshape the coastline and seabed. These morphodynamic effects of a storm are not clear. The output of our storm surge model contains surface elevation as well as flow velocity. By coupling the flow velocity and sediment transport, the model can be extended to take into account the morphodynamic evolution, allowing the study of the immediate morphodynamic effects of a storm.

- Future research using this model should improve the open boundary condition. As discussed in chapter 4, the model results of storm surge in New Orleans coastal basin showed a periodic signal, which is due to the reflecting effect of Dirichlet boundary condition. One approach to improve it is to apply the nonreflecting boundary condition introduced in chapter 3, which allows the wave generated inside the basin to freely propagate away. 


\section{Bibliography}

Abbott PL (1996) Natural Disasters, 8th edn. Wm. C. Brown Publishing Co., New York

Abraham G (1960) Hurricane storm surge considered as a resonance phenomenon. Coast Eng Proceedings 1(7):31, DOI 10.9753/icce.v7.31

Arthur PN (1964) The relation of wind and pressure to extratropical storm surges at Atlantic city. J Appl Meteor pp 155-163, DOI 10.1175/1520-0450(1964)003<0155: TROWAP $\rangle 2.0 . \mathrm{CO} ; 2$

Bertin X, Bruneau N, Breilh JF, Fortunato AB, Karpytchev M (2012) Importance of wave age and resonance in storm surges: The case Xynthia, Bay of Biscay. Ocean Model 42(0):16-30, DOI 10.1016/j.ocemod.2011.11.001

Birchfield GE (1967) Horizontal transport in a rotating basin of parabolic depth profile. J Geophys Res 72(24):6155-6163, DOI 10.1029/JZ072i024p06155

Birchfield GE (1969) Response of a circular model Great Lake to a suddenly imposed wind stress. J Geophys Res 74(23):5547-5554, DOI 10.1029/JC074i023p05547

Blain CA, Westerink JJ, Luettich RA (1994) The influence of domain size on the response characteristics of a hurricane storm surge model. J Geophys Res 99(C9):18,467-18,479, DOI 10.1029/94JC01348

Blake ES, Cobb HD, Roberts DP (2006) Tropical cyclone report Hurricane Wilma 15-25 October 2005. Report, National Hurricane Center

Blake ES, Kimberlain TB, Berg RJ, Cangialosi JP, II JLB (2013) Tropical cyclone report Hurricane Sandy 22-29 February 2013. Report, National Hurricane Center

Borne K (1998) Observational study of sea and land breeze on the Swedish West coast with focus on an Archipelago, vol 34. Earth Science Centre, Göteborg University

Bossak BH (2005) Katrina's wake. Eos Trans Amer Geophys 8627:333-334, DOI 10.1029/2005EO370003

Boyd JP (2000) Chebyshev and Fourier Spectral Methods, 2nd edn. Dover, New York

Breaker LC, Tseng Y, Wang X (2010) On the natural oscillations of Monterey Bay: Observations, modeling, and origins. Prog Oceanogr 86(34):380-395, DOI 10.1016/ j.pocean.2010.06.001

Bunpapong M, Reid RO, Whitaker RE (1985) An investigation of hurricane-induced forerunner surge in the Gulf of Mexico. Springfield, Va. : Available from National Technical Information Service, Vicksburg, Mississippi 
Bunya S, Dietrich JC, Westerink JJ, Ebersole BA, Smith JM, Atkinson JH, Jensen R, Resio DT, Luettich RA, Dawson C, Cardone VJ, Cox AT, Powell MD, Westerink HJ, Roberts HJ (2010) A high-resolution coupled riverine flow, tide, wind, wind wave, and storm surge model for Southern Louisiana and Mississippi. part I: Model development and validation. Mon Wea Rev 138:345-377, DOI 10.1175/2009MWR2906.1

Büsching F (2003) Storm wave resonance controlled by hollow block structure. In: Proc. 6th Int. Conf. on Coastal and Port Engineering in Developing Countries COPEDEC VI, Colombo, Sri Lanka., pp 1-20

Callaghan J (1997) The South Pacific and Southeast Indian Ocean tropical cyclone season 1995-96. Aust Met Mag 46:325-339

Chen Q, Wang L, Tawes R (2008) Hydrodynamic response of Northeastern Gulf of Mexico to hurricanes. Estuaries Coasts 31(6):1098-1116, DOI 10.1007/ s12237-008-9089-9

Chen WL, Roos PC, Schuttelaars HM, Hulscher SJMH (2015) Resonance properties of a closed rotating rectangular basin subject to space- and time-dependent wind forcing. Ocean Dyn 65(3):325-339, DOI 10.1007/s10236-015-0813-2

Colle BA, Buonaiuto F, Bowman MJ, Wilson RE, Flood R, Hunter R, Mintz A, Hull D (2008) New York city's vulnerability to coastal flooding. Bull Amer Meteor Soc 89:829-841, DOI 10.1175/2007BAMS2401.1

Corps of Engineers (1970) Hurricane Camille 14-22 August 1969. Tech. rep., U. S. Army Engineer district, Mobile, Corps of Engineers, Mobile, Alabama

Craig PD (1989) Constant-eddy-viscosity models of vertical structure forced by periodic winds. Cont Shelf Res 9(4):343-358, DOI 10.1016/0278-4343(89)90038-1

Csanady GT (1968a) Wind-driven summer circulation in the Great Lakes. J Geophys Res 73(8):2579-2589, DOI 10.1029/JB073i008p02579

Csanady GT (1968b) Wind-induced barotropic motions in long lakes. J Phys Oceanogr 3(4):429-438, DOI 10.1175/1520-0485(1973)003〈0429:WIBMIL $\rangle$ 2.0.CO;2

Csanady GT (1982) Circulation in the Coastal Ocean. Reidel, Dordrecht, Netherlands

Dietrich JC, Tanaka S, Westerink JJ, Dawson CN, Luettich RA, Zijlema M, Holthuijsen LH, Smith JM, Westerink LG, Westerink HJ (2012) Performance of the unstructured-mesh, SWAN+ADCIRC model in computing hurricane waves and surge. J Sci Comput 52(2):468-497, DOI 10.1007/s10915-011-9555-6

Doodson AT (1924) Meteorological perturbations of sea-level and tides. Geophys J Int 1:124-147, DOI 10.1111/j.1365-246X.1924.tb05363.x

Dowdeswell JA, Benham TJ (2003) A surge of Perseibreen, Svalbard, examined using aerial photography and ASTER high resolution satellite imagery. Polar Res 22(2):373-383, DOI 10.1111/j.1751-8369.2003.tb00118.x

Eckart C (1951) Surface waves in water of variable depth. Tech. Rep. 100, Scripps

Ekman VW (1905) On the influence of the earth's rotation on ocean-currents. Ark Mat Ast Fys 2:11:52 
Fanelli P, Wolcott D (2012) Hurricane Isaac. Tech. rep., National Oceanic and Atmospheric Administration (NOAA), NOAA water level and Meteorological Data Report

Garratt JR (1977) Review of drag coefficients over oceans and continents. Mon Wea Rev 105:915-929, DOI 10.1175/1520-0493(1977)105〈0915:RODCOO $\rangle$ 2.0.CO;2

Gill AE (1982) Atmosphere-Ocean Dynamics. Academic Press, London

Gockenbach MS (2006) Understanding and implementing the Finite Element Method. Society of industrial and applied mathematics (SIAM), Philadelphia

Greenspan HP (1956) The generation of edge waves by moving pressure distributions. J Fluid Mech 1:574-592, DOI 10.1017/S002211205600038X

Hanley M, Hoggart S, Simmonds D, Bichot A, Colangelo M, Bozzeda F, Heurtefeux H, Ondiviela B, Ostrowski R, Recio M, Trude R, Zawadzka-Kahlau E, Thompson R (2014) Shifting sands? Coastal protection by sand banks, beaches and dunes. Coast Eng 87(0):136-146, DOI 10.1016/j.coastaleng.2013.10.020

Holland GJ (1980) An analytic model of the wind and pressure profiles in hurricanes. Mon Wea Rev 108(8):1212-1218, DOI 10.1175/1520-0493(1980)108<1212: AAMOTW $>2.0 . \mathrm{CO} ; 2$

Hough P (2008) Understanding Global security. Routledge, New York

Irish JL, Resio DT, Ratcliff JJ (2008) The influence of storm size on hurricane surge. J Phys Oceanogr 38(9):2003-2013, DOI 10.1175/2008JPO3727.1

James L (1998) Fluid mechanics of Tropical Cyclones. Theo Comp Fluid Dyn 10(14):3-21, DOI 10.1007/s001620050048

Jelesnianski CP, Chen J, Shaffer WA (1992) SLOSH: Sea, lake, and overland surges from hurricanes. Tech. Rep. NWS 48, NOAA

Kennedy AB, Gravois UZ, Brian C, Westerink JJ, Hope ME, Dietrich JC, Powell MD, Cox AT, Luettich RA, Dean RG (2011) Origin of the Hurricane Ike forerunner surge. Geophys Res Lett 38(8), DOI 10.1029/2011GL047090

Klaver EN, Vrijling JK, Jonkman SN, van Gelder PHAJM, Holthuijsen LH, Takahashi S, Kawai H (2006) Probabilistic analysis of typhoon induced hydraulic boundary conditions for suo-nada bay. In: Coast. Eng. 2006, World Scientific, pp 791-801, DOI 10.1142/9789812709554_0068

Kumar M, Schuttelaars HM, Roos PC, Möller M (submitted) Three-dimensional semiidealized model for tidal motion in tidal estuaries. An application to the Ems estuary

Lapidez JP, Tablazon J, Dasallas L, Gonzalo LA, Cabacaba KM, Ramos MMA, Suarez JK, Santiago J, Lagmay AMF, Malano V (2015) Identification of storm surge vulnerable areas in the Philippines through the simulation of Typhoon Haiyan-induced storm surge levels over historical storm tracks. Nat Hazard Earth Sys 3(2):919-939, DOI 10.5194/nhessd-3-919-2015 
Libicki C, Bedford KW (1990) Sudden, extreme Lake Erie storm surges and the interaction of wind stress, resonance, and geometry. J Great Lake Res 16(3):380-395, DOI 10.1016/S0380-1330(90)71432-3

Lin N, Emanuel KA, Smith JA, Vanmarcke E (2010) Risk assessment of hurricane storm surge for new york city. J Geophys Res 115, DOI 10.1029/2009JD013630

Lin N, Emanueland K, Vanmarcke E (2014) Hurricane analysis: a review on the physically-based approach. In: Deodatis G, Ellinwood BR, Frangopol DM (eds) Safety, reliability, risk and Life-Cycle performance of structures ans infrastructures, pp 1291-1297, DOI 10.1201/b16387-191

Lorentz HA (1922) Het in rekening brengen van den weerstand bij schommelende vloeistofbewegingen. De Ingenieur 37:695, in Dutch

Lum T, Margesoon R (2014) Typhoon Haiyan (Yolanda): U.S. and international response to Philippines disaster. Report R43309, Congressional Research Service

Madsen H, Jakobsen F (2004) Cyclone induced storm surge and flood forecasting in the northern Bay of Bengal. Coast Eng 5(4):277-296, DOI 10.1016/j.coastaleng. 2004.03.001

Mathieu PP, Deleersnijder E, Cushman-Roisin B, Beckers JM, Bolding K (2002) The role of topography in small well-mixed bays, with application to the Lagoon of Mururoa. Cont Shelf Res 22(9):1379-1395, DOI 10.1016/S0278-4343(02)00002-X

Mohammed-Zaki MA (1980) Time scales in wind-driven lake circulations. J Geophys Res 85(C3):1553-1562, DOI 10.1029/JC085iC03p01553

Moon IJ, Oh IS, Murty T, Youn YH (2003) Causes of the unusual coastal flooding generated by typhoon Winnie on the west coast of Korea. Natural Hazards 29(3):485-500, DOI 10.1023/A:1024798718572

Murck BW, Skinner BJ, Porter SC (1997) Dangerous Earth: an introduction to geologic hazards. Wiley, New York

National Oceanic, Atmospheric Administration (2015) tides and currents. URL http: //www.tidesandcurrents.noaa.gov/

Pasche RJ, Brown DP, Blake ES (2005) Tropical cyclone report: Hurricane Charley 9-14 August 2004. Report, National Hurricane Center

Pita G, Pinelli JP, Cocke S, Gurey K, Mitrani-Reiser J, Weekes J, Hamid S (2012) Assessment of hurricane-induced internal damage to low-rise buildings in the Florida public hurricane loss model. J Wind Eng Ind Aerodyn 104-106:76-87, DOI 10.1016/ j.jweia.2012.03.023

Ponte AL (2010) Periodic wind-driven circulation in an elongated and rotating basin. J Phys Oceanogr 40(9):2043-2058, DOI 10.1175/2010JPO4235.1

Ponte AL, de Velasco GG, Valle-Levinson A, Winters KB, Winant CD (2012) Winddriven subinertial circulation inside a semienclosed bay in the Gulf of California. J Phys Oceanogr 42(6):940-955, DOI 10.1175/JPO-D-11-0103.1 
Proudman J (1929) The effects on the sea of changes in atmospheric pressure. Mon Not R Astr Soc, Geophys Suppl 2:197

Proudman J (1953) Dynamical Oceanography. Wiley, New York

Pugh DT (1987) Tides, Surges and Mean Sea-level. Wiley, New York

Rabinovich AB (2009) Seiches and harbor oscillations. In: Handbook of Coastal and Ocean Engineering, SpringerBriefs in Environmental Science, World Scientific, chap 9, pp 193-236

Rao DB (1966) Free gravitational oscillations in rotating rectangular basins. J Fluid Mech 25(3):523-555, DOI 10.1017/S0022112066000235

Redfield AC, Miller AR (1957) Water levels accompanying Atlantic Coast Hurricanes. American Meteorological Society

Rego JL, Li C (2009) On the importance of the forward speed of hurricane in storm surge forecasting: A numerical study. Geophys Res Lett 36(7), DOI 10.1029/2008GL036953

Resio DT, Westerink JJ (2008) Modelling the physics of storm surges. Physics today 61 , DOI $10.1063 / 1.2982120$

Roos PC, Schuttelaars HM (2011) Influence of topography on tide propagation and amplification in semi-enclosed basins. Ocean Dyn 61(1):21-38, DOI 10.1007/ s10236-010-0340-0

Roos PC, Velema JJ, Hulscher SJMH, Stolk A (2011) An idealized model of tidal dynamics in the North Sea: resonance properties and response to large-scale changes. Ocean Dyn 61(12):2019-2035, DOI 10.1007/s10236-011-0456-x

Schultz DM, Vaughan G (2011) Occluded fronts and the occlusion process: A fresh look at conventional wisdom. Bull Amer Meteor Soc 92:443-466, DOI 10.1175/ 2010BAMS3057.1

Simpson R (1974) The hurricane disaster-potential scale. Weatherwise 27:169-186, DOI 10.1080/00431672.1974.9931702

Smith SD, Anderson RJ, Oost WA, Kraan C, Maat N, Cosmo JD, Katsaros KB, Davidson KL, Bumke K, Hasse L, Chadwick HM (1992) Sea surface wind stress and drag coefficients: The HEXOS results. BoundLayer Meteor 60(1-2):109-142, DOI 10.1007/BF00122064

Sobey RJ (2006) Normal mode decomposition for identification of storm tide and tsunami hazard. Coast Eng 53(23):289-301, DOI 10.1016/j.coastaleng.2005.10.016

Tannehill IR (1956) Hurricanes. Princeton University Press, Princeton, New Jersey

Thiebaut S, Vennell R (2011) Resonance of long waves generated by storms obliquely crossing shelf topography in a rotating ocean. J Fluid Mech 682:261-288, DOI $10.1017 /$ jfm.2011.221 
Tomkratoke S, Sirisup S, Udomchoke V, Kanasut J (2015) Influence of resonance on tide and storm surge in the Gulf of Thailand. Cont Shelf Res 109:112-126, DOI $10.1016 /$ j.csr.2015.09.006

Vennell R (2010) Resonance and trapping of topographic transient ocean waves generated by a moving atmospheric disturbance. J Fluid Mech 650:427-442, DOI $10.1017 /$ S0022112009993739

Verlaan M, Zijderveld A, de Vries JW, Kroos J (2005) Operational storm surge forecasting in the Netherlands: developments in the last decade. Phil Trans R Soc A 363(1831):1441-1453, DOI 10.1098/rsta.2005.1578

Vickery PJ, Wadhera D (2008) Statistical models of Holland pressure profile parameter and radius to maximum winds of hurricanes from flight-level pressure and $\mathrm{H}^{*}$ wind data. J Appl Meteorol Clim 47:2497-2517, DOI 0.1175/2008JAMC1837.1

Weaver R, Slinn D (2010) Influence of bathymetric fluctuations on coastal storm surge. Coast Eng 57(1):62-70, DOI 10.1016/j.coastaleng.2009.09.012

Weisberg RH, Zheng L (2006) Hurricane storm surge simulations for Tampa Bay. Estuaries Coasts 29(6):899-913, DOI 10.1007/BF02798649

Westerink JJ, Luettich RA, Feyen JC, Atkinson JH, Dawson C, Roberts HJ, Powell MD, Dunion JP, Kubatko EJ, Pourtaheri H (2008) A basin- to channel-scale unstructured grid hurricane storm surge model applied to Southern Louisiana. Mon Wea Rev 136:833-864, DOI 10.1175/2007MWR1946.1

Whitham GB (1979) Lectures on Wave propagation. Springer-Verlag, New York

Wilson BW (1972) Seiches. Adv Hydrosci 8:1-94

Winant CD (2004) Three-dimensional wind-driven flow in an elongated, rotating basin. J Phys Oceanogr 34(2):462-476, DOI 10.1175/1520-0485(2004)034〈0462:TWFIAE〉 2.0.CO;2

Wu J (1980) Wind-stress coefficients over sea surface near neutral conditions-A revisit. J Phys oceanogr 10:727-740, DOI 10.1175/1520-0485(1980)010<0727:WSCOSS $\rangle 2.0$. $\mathrm{CO} ; 2$

Wu J (1982) Wind-stress coefficients over sea surface from sea breeze to hurricane. J Geophys Res 87(C9):9704-9706, DOI 10.1029/JC087iC12p09704

Yankovsky AE (2009) Large-scale edge waves generated by hurricane landfall. J Geophys Res 114:2156-2202, DOI 10.1029/2008JC005113 


\section{List of publications}

1. Chen WL, Roos PC, Schuttelaars HM, Hulscher SJMH (2015) Resonance properties of a closed rotating rectangular basin subject to space- and time-dependent wind forcing. Ocean Dyn 65(3):325-339, DOI 10.1007/s10236-015-0813-2

2. Chen WL, Roos PC, Schuttelaars HM, Kumar M, Zitman TJ, Hulscher SJMH (submitted) Response of large-scale coastal basins to wind forcing: influence of topography.

3. Chen WL, Straatsma L, Roos PC, Schuttelaars HM, Van Ledden M, Hulscher SJMH (submitted) The influence of storm characteristics on storm surges in the New Orleans coastal basin.

4. Ndungu JN, Chen WL, Augustijn DCM, Hulscher, SJMH. (2015) Analysis of the driving force of hydrodynamics in Lake Naivasha, Kenya. Open Journal of Modern Hydrology 5:95-104, DOI 10.4236/ojmh.2015.54009

5. Chen WL, Roos PC, Schuttelaars HM, Hulscher SJMH (2014). Storm surge response in large-scale basins: influence of topography. In C.A.F. Schettini(Ed.) Book of abstract of the 17th Physics of Estuaries and Coastal Seas (PECS) conference, Porto de Galinhas, Pernambuco, Brazil, 19-24 October 2014 (pp.14). Pernambuco: Center of Geosciences \& Engineering, Federal University of Pernambuco.

6. Chen WL, Roos PC, Schuttelaars HM, Hulscher SJMH (2014). Resonance properties of a closed rotating rectangular basin subject to periodic wind forcing, In M.S. Ab Razak, L. Sembiring \& M. van der Wegen (Eds.), Book of abstracts NCK-Days 2014, 27-28 March 2014, Delft: UNESCO-IHE.

7. Chen WL, Roos PC, Schuttelaars HM, Hulscher SJMH(2013). Sand extraction and coastal safety. In: 2013 BURGERSDAG, University of Twente. JM Burgerscentrum.

8. Chen WL, Roos PC, Schuttelaars HM, Hulscher SJMH(2012). Modelling the competition between wind-driven and density-driven circulation in the Ems estuary. In: The physics of Estuaries and Coastal Seas (PECS) Symposium, New York, USA (pp. 16-17). 


\section{About the author}

Wenlong Chen was born in Zhejiang Province, China, on $12^{\text {th }}$ Juanuary 1988. From 2002 to 2005, he attended 'Jiang Shan senior high school' in JiangShan, ZheJiang Province. Straight after graduation, he started his academic study in Water Resource and Ocean Engineering at ZheJiang University. In 2009, he obtained his BSc degree with a graduation project on the sediment dynamics of a dredged channel and was awarded with Outstanding graduate of ZheJiang University. This graduation project was supervised by Prof. Dr. Z.Sun and leaded to a thesis entitled 'Deposition rate in dredged channel'.

From 2009 to 2011, he studied Ocean Construction and Environment as a PhD candidate of ZheJiang University. After completed the education required for a $\mathrm{PhD}$ degree he participated in a consultation project named 'Strategic research on the exploitation of coast and island in ZheJiang Provive'. He also continued the study on the deposition rate of dredged channel and participated in a project named 'Key technology in the exploitation of a large scale coastal bay, predicting the deposition rate in a navigation channel'.

In 2011, he got the opportunity to work on the $\mathrm{PhD}$ project 'Sand extraction and coastal safety' in the Water Engineering and Management group at the University of Twente. He studied storm surge processes in large-scale coastal basins, particularly focusing on the influences of geometry, topography and storm characteristics on the water levels along the coast. This work resulted in the present thesis. 